\title{
ERGODIC THEORY FOR MARKOV FIBRED SYSTEMS AND PARABOLIC RATIONAL MAPS
}

\author{
JON AARONSON, MANFRED DENKER, AND MARIUSZ URBAŃSKI
}

\begin{abstract}
A parabolic rational map of the Riemann sphere admits a nonatomic $h$-conformal measure on its Julia set where $h=$ the Hausdorff dimension of the Julia set and satisfies $1 / 2<h<2$. With respect to this measure the rational map is conservative, exact and there is an equivalent $\sigma$-finite invariant measure. Finiteness of the measure is characterised. Central limit theorems are proved in the case of a finite invariant measure and return sequences are identified in the case of an infinite one. A theory of Markov fibred systems is developed, and parabolic rational maps are considered within this framework.
\end{abstract}

\section{INTRODUCTION}

In this paper we study parabolic rational maps on $\overline{\mathbb{C}}$, the Riemann sphere. More specifically, we consider the ergodic theory of such maps with respect to conformal measures on their Julia sets. The nonsingular transformations involved turn out to be Markov fibred systems. We develop a theory of Markov fibred systems based on, and generalising [33], which also applies to some Markov shifts and Markov maps of the interval.

A rational map $T: \overline{\mathbb{C}} \rightarrow \overline{\mathbb{C}}$ of degree $\geq 2$ is called parabolic if the restriction $\left.T\right|_{J(T)}$ is expansive but not expanding in the spherical metric on $\overline{\mathbb{C}}$ (see [14]). Equivalently (see [15]), $J(T)$ contains no critical point of $T$, but contains rationally indifferent periodic points. For example, a Blaschke product (i.e., a rational inner function on the unit disc (see [1])) with an indifferent fixed point is a parabolic rational map whose Julia set is contained in the unit circle $S^{1}$ (§9).

Let $t \geq 0$. A probability measure $m$ on $J(T)$ is called $t$-conformal for $T:: \overline{\mathbb{C}} \rightarrow \overline{\mathbb{C}}$ if

$$
m(T(A))=\int_{A}\left|T^{\prime}\right|^{t} d m
$$

for every Borel set $A \subset J(T)$ such that $\left.T\right|_{A}$ is injective (see [34]).

It turns out (Theorem 8.7) that, for $h=$ the Hausdorff dimension of $J(T)$, the unique $h$-conformal measure for $T$ is nonatomic. This result is obtained

Received by the editors December 4, 1990 and, in revised form, January 17, 1991.

1980 Mathematics Subject Classification (1985 Revision). Primary 28D05; Secondary 30C99, $58 \mathrm{~F} 11,58 \mathrm{~F} 12,60 \mathrm{~F} 05$.

The first and third authors' research was partially supported by Deutsche Forschungsgemeinschaft SFB 170, Göttingen.

The second author's research was partially supported by School of Mathematics, Tel Aviv University. 
(Theorem 8.4) by analysing the asymptotic behaviour of inverse branches in small neighbourhoods of indifferent periodic points using the flower theorem. This analysis also gives improved estimates for $h$ (Theorems 8.5 and 8.8), in particular $1 / 2<h<2$. Existence of an $h$-conformal measure was established in [15], and it was shown in [14] to be unique, if nonatomic.

Let $T: \overline{\mathbb{C}} \rightarrow \overline{\mathbb{C}}$ be a parabolic rational map and $m$ its $h$-conformal measure. It is known (see [14]), that there is a topological Markov partition (see [31])) with respect to which $(J(T), m, T)$ is a fibred system. Also (see [14]), $T$ is ergodic and has a $\sigma$-finite invariant measure $\mu$ equivalent to $m$. We show in addition that $T$ is conservative and exact, and characterise finiteness of $\mu$ in terms of $h$ and the Taylor expansion around indifferent periodic points.

For $\omega$ a rationally indifferent periodic point and $q \in \mathbb{N}$ such that $T^{q}$ fixes $\omega$ with unit derivative, the Taylor expansion of $T^{q}$ around $\omega$ is of the form $z+a(z-\omega)^{p+1}+\cdots$, where $p=p(\omega)=p(\omega, q)$ does not depend on $q$. Let

$$
\alpha=\min _{\omega} \frac{p(\omega)+1}{p(\omega)} h .
$$

Because of the existence of the nonatomic $h$-conformal measure we obtain $1<\alpha<4$. The invariant measure $\mu$ is finite if and only if $\alpha>2$. For example, if $h \leq 1$ (in particular for the parabolic Blaschke products) then $\mu$ is infinite. For the polynomials $z \mapsto z+z^{2}, z \mapsto z-z^{2}$, and $z \mapsto z^{2}+1 / 4$ the measure $\mu$ is finite (see $\S 9$ ).

In case the measure is finite we derive the central limit theorem for partial sums of Hölder continuous functions which vanish on neighbourhoods of the rationally indifferent periodic points (Theorem 9.12). This is derived using weak dependence structures of an auxiliary transformation (the jump transformation of [33]).

In case the measure is infinite then $T$ has Darling-Kac sets with continued fraction mixing return time processes and return sequences of the form $\left\{n^{\alpha-1}\right\}_{n}$ for $1<\alpha<2$ and $\left\{\frac{n}{\log n}\right\}_{n}$ for $\alpha=2$ (Theorem 9.11). The existence of Darling-Kac sets, and regularly varying return sequence implies (see $[2,3])$ convergence in distribution of normalized ergodic sums, and, in case the return time process is continued fraction mixing, we also have (see [4-6]) laws of the iterated logarithm for the ergodic sums. These and additional results are stated in detail (with definitions) in $\S 1$, which is a brief overview of the relevant ergodic theory for nonsingular transformations.

Fibred systems have been studied in [33] and [36] with application to piecewise onto transformations of the interval and multidimensional versions have been studied in [38]. As mentioned above, parabolic rational maps are Markov fibred systems (see [14]), as are Markov shifts and Markov maps of the interval. In $\S \S 2-7$ we develop the basic ergodic theory of these maps, extending [33] and [36], using Schweiger's relaxation of the Renyi property (see [30]).

An irreducible Markov fibred system with the Schweiger property is either totally dissipative or conservative ergodic (Theorem 2.5). A conservative, irreducible Markov fibred system with the Schweiger property has a $\sigma$-finite invariant measure and Darling-Kac sets with continued fraction mixing return time processes (Theorem 3.1), has wandering rates (Lemma 4.2), and is exact, if aperiodic (Theorem 3.2).

The fibred systems corresponding to parabolic rational maps (parabolic fi- 
bred systems) are studied in $\S \S 5-7$. Proposition 5.6 provides the information necessary to use the analysis of the asymptotic behaviour of inverse branches in small neighbourhoods of indifferent periodic points using the flower theorem to identify wandering rates. Whence the characterisation of finiteness of the invariant measure, and calculation of the return sequences in case this measure is infinite. In $\S \S 6$ and 7 we prove central limit theorems for aperiodic, parabolic fibred systems with finite invariant measures.

In $\S 9$, we show that parabolic rational maps are indeed parabolic fibred systems (Theorem 9.1). This enables application of $\S \S 5-7$ for parabolic rational maps, except for two last things: continuity of the invariant density for the jump transformation and continued fraction mixing with exponential rates. These last two things are provided by Theorem 9.6.

\section{ERGODIC THEORY OF NONINVERTIBLE TRANSFORMATIONS}

In this section, for the convenience of the reader, we collect without proof some results on noninvertible nonsingular transformations of a Lebesgue probability space $(X, \mathscr{F}, m)$. The proofs of most of the results can be found in $[29,2-7]$.

Let $T: X \rightarrow X$ be a measurable transformation (that is, $T^{-1} A \in \mathscr{F} \forall A \in$ $\mathscr{F}$ ). The transformation $T$ is called nonsingular if $m \circ T^{-1} \sim m$, that is, for $A \in \mathscr{F}$,

$$
m\left(T^{-1} A\right)=0 \Leftrightarrow m(A)=0 .
$$

The transformation $T$ is called nonsingular on $A \in \mathscr{F}$ if $T A \in \mathscr{F}$ and, for $B \in \mathscr{F} \cap T A$,

$$
m\left(A \cap T^{-1} B\right)=0 \Leftrightarrow m(B)=0 .
$$

The transformation $T$ is called invertible on $A \in \mathscr{F}$ if $T$ is $1-1$ on $A$, $T A \in \mathscr{F}$, and $T^{-1}: T A \rightarrow A$ is measurable. The transformation $T$ is called invertible if $T$ is invertible on $X$.

If $T$ is nonsingular and invertible, then clearly so is $T^{-1}$.

Suppose that $T$ is a nonsingular transformation of $X$. A set $W \in \mathscr{F}$ is called a wandering set (for $T$ ) if the sets $\left\{T^{-n} W\right\}_{n=0}^{\infty}$ are disjoint. It is not hard to show using an exhaustion argument that there is a set $X_{d} \in \mathscr{F}$ with the properties

$$
\forall A \in \mathscr{F} \cap X_{d}, \quad m(A)>0,
$$

$\exists$ a wandering set $W \subseteq A \ni m(W)>0$,

and

$$
W \in \mathscr{F} \text { a wandering set } \Rightarrow m\left(W \backslash X_{d}\right)=0 .
$$

The set $X_{d}$ is uniquely defined up to measure zero. It also satisfies $T^{-1} X_{d} \subseteq$ $X_{d} \bmod m$, since, if $W$ is a wandering set then so is $T^{-1} W$. The set $X_{d}$ is called the dissipative part of $T$, and in case $m\left(X \backslash X_{d}\right)=0$, the transformation $T$ is called (totally) dissipative.

The set $X_{c}:=X \backslash X_{d}$ is called the conservative part of $T$, and $T$ is called conservative if $m\left(X_{d}\right)=0$. The set $X_{c}$ satisfies $T^{-1} X_{c} \supseteq X_{c} \bmod m$. It can be shown that for a set $A \in \mathscr{F} m(A)>0$,

$$
A \subseteq X_{c} \bmod m \Leftrightarrow \sum_{n=1}^{\infty} 1_{B} \circ T^{n}=\infty \text { a.e. on } B \quad \forall B \in \mathscr{F} \cap A, m(B)>0,
$$


and that $T: X_{c} \rightarrow X_{c}$ is a (conservative) nonsingular transformation (when $\left.m\left(X_{c}\right)>0\right)$.

The conservative part of a power of $T$ is the same as that of $T \bmod m$.

If $\exists q \in \mathscr{P}(X), q \circ T^{-1}=q \sim m$, then clearly there can be no wandering sets with positive measure, whence $m\left(X_{d}\right)=0$ and $T$ is conservative. Here, and throughout, $\mathscr{P}(X)$ denotes the collection of probabilities on $(X, \mathscr{F})$.

In case $T$ is invertible, the conservative and dissipative parts of $T$ can be chosen to be $T$-invariant. For noninvertible, nonsingular transformations, this is not necessarily the case, as illustrated by the following

Example. Let $X=[0,1), m$ be Lebesgue measure, and $T: X \rightarrow X$ be defined by

$$
T x= \begin{cases}2 x, & x \in[0,1 / 4), \\ 2 x-1 / 2, & x \in[1 / 4,1 / 2), \\ 2 x-1, & x \in[1 / 2,1) .\end{cases}
$$

Here, $X_{c}=[0,1 / 2]$ and $X_{d}=[1 / 2,1] \bmod m$.

A nonsingular transformation $T$ is called ergodic if $A \in \mathscr{F}$ and $T^{-1} A=A$ mod $m$ implies $m(A) m\left(A^{c}\right)=0$. It is not hard to show that if $\left.T\right|_{X_{c}}$ is ergodic, then

$$
T \text { is ergodic } \Leftrightarrow \bigcup_{n=1}^{\infty} T^{-n} X_{c}=X \quad \bmod m .
$$

In the example, it is well known that $\left.T\right|_{X_{c}}$ is ergodic, and, evidently,

$$
\bigcup_{n=1}^{\infty} T^{-n} X_{c}=X \quad \bmod m
$$

so $T$ is ergodic.

An invertible ergodic nonsingular transformation of a nonatomic measure space is necessarily conservative. The example shows that this is not necessarily the case for noninvertible, nonsingular transformations.

If $T$ is a conservative, ergodic nonsingular transformation and $\mu \ll m$ is a $\sigma$-finite $T$-invariant measure on $X$, then $\mu \sim m$. For nonconservative, ergodic transformations, this is not necessarily the case, as illustrated by the example, with Lebesgue measure restricted to the conservative part $(0,1 / 2)$ being $T$-invariant.

If $T: X \rightarrow X$ is nonsingular then $f \rightarrow f \circ T$ defines a linear isometry of $L^{\infty}(m)$ whose dual preserves $L^{1}(m)$. The restriction of this dual to $L^{1}(m)$ is known as the dual, or Frobenius-Perron or transfer operator, denoted by $\widehat{T}$, and defined by

$$
\int_{X} \widehat{T} f \cdot g d m=\int_{X} f \cdot g \circ T d m .
$$

Note that the domain of definition of $\widehat{T}$ can be extended to all nonnegative measurable functions by the above formula.

It is not hard to see that for $f \in L^{1}(m), f>0$ a.e.

$$
\left\{x \in X: \sum_{n=1}^{\infty} \widehat{T}^{k} f(x)=\infty\right\}=X_{c} \bmod m .
$$


Also,

$T$ is conservative and ergodic

$$
\Leftrightarrow \sum_{n=0}^{\infty} \widehat{T}^{n} f=\infty \text { a.e. } \forall f \in L^{1}(m), f \geq 0 \text { a.e., } \int_{X} f d m>0 \text {, }
$$

and, if $\mu \ll m$ and $\frac{d \mu}{d m}=h$, then

$$
\mu \circ T^{-1}=\mu \Leftrightarrow \widehat{T} h=h .
$$

A nonsingular transformation $T: X \rightarrow X$ is called exact if

$$
A \in \bigcap_{n=1}^{\infty} T^{-n \mathscr{F}} \Rightarrow m(A) m\left(A^{c}\right)=0
$$

Suppose $T$ is conservative and nonsingular, and let $A \in \mathscr{F}$, then $m$-a.e. point of $A$ returns infinitely often to $A$ under iterations of $T$, and in particular the return time function, defined for $x \in A$ by $\varphi(x):=\min \left\{n \geq 1: T^{n} x \in A\right\}$, is finite $m$-a.e. on $A$.

The induced transformation on $A$ (see [27]) is defined by

$$
T_{A} x=T^{\varphi(x)} x \text {. }
$$

It is shown in [27] that $T_{A}$ is a nonsingular transformation of $\left(A, \mathscr{F} \cap A,\left.m\right|_{A}\right)$, and that

$$
\begin{gathered}
T \text { ergodic } \Rightarrow T_{A} \text { ergodic, } \\
T_{A} \text { ergodic and } \bigcup_{n=1}^{\infty} T^{-n} A=X \bmod m \Rightarrow T \text { ergodic. }
\end{gathered}
$$

If, in the above situation, $\left.q \ll m\right|_{A}$ is a $T_{A}$-invariant measure, and the measure $\mu$ is defined on $X$ by

$$
\mu(B)=\sum_{k=0}^{\infty} q\left(A \cap T^{-k} B \backslash \bigcup_{j=1}^{k} T^{-j} A\right)=\int_{A} \sum_{k=0}^{\varphi-1} 1_{B} \circ T^{k} d q,
$$

then (see [26]), $\mu \ll m$ and $\mu \circ T^{-1}=\mu$. The above formula is known as Kac's formula.

Let $(X, \mathscr{F}, \mu, T)$ be a conservative ergodic measure preserving transformation on a $\sigma$-finite nonatomic measure space. Let $\widehat{T}$ be the dual operator. A set $A \in \mathscr{F}$ with $0<\mu(A)<\infty$ is called a Darling-Kac set for $T$ if there exist constants $\left\{a_{n}>0\right\}_{n \geq 1}$ such that

$$
\lim _{n \rightarrow \infty} \frac{1}{a_{n}} \sum_{k=1}^{n} \widehat{T}^{k} 1_{A}=\mu(A)
$$

uniformly on $A$.

In this situation, it follows that (see [3, pp. 1044-1045]) the system is also pointwise dual ergodic:

There exist constants $a_{n}=a_{n}(T),(n \geq 1)$, such that

$$
\lim _{n \rightarrow \infty} \frac{1}{a_{n}(T)} \sum_{k=0}^{n-1} \widehat{T}^{k} f=\int_{X} f d \mu \text { a.e. }
$$

for any $f \in L^{1}(X, \mathscr{F}, \mu, T)$. 
The sequence $a_{n}(T)$ is uniquely determined up to asymptotic equality, and is called the return sequence of $T$. In case the return sequence turns out to be regularly varying with index $\alpha \in[0,1]$, the asymptotic behaviour of

$$
S_{n}(f)=\sum_{k=0}^{n-1} f \circ T^{k}
$$

can be described almost surely and in distribution as follows:

Theorem [7, Theorem 1]. Assume that $a_{n}(T)=n^{\alpha} h(n)$ is regularly varying with index $\alpha \in(0,1]$ or $\alpha=0$ and with $h(n) \sim \exp \left(\int_{1}^{t} \frac{\eta(t)}{t} d t\right)$, where $\eta$ is monotonic, $|\eta(t)| \downarrow 0$ as $t \uparrow \infty$, and $|\eta(t)| / \log t \rightarrow \infty$ as $t \rightarrow \infty$. Then for any function $f \in L^{1}(\mu)$

$$
\lim _{N \rightarrow \infty} \frac{1}{\log N} \sum_{n=1}^{N} \frac{1}{n a_{n}(T)} S_{n}(f)=\int_{X} f d \mu \quad \text { in measure. }
$$

Theorem [2, Theorem 1]. Let $(X, \mathscr{F}, \mu, T)$ be pointwise dual ergodic with regularly varying return sequence $a_{n}(T)$ of index $\alpha \in[0,1]$. Then for any $f \in L_{+}^{1}(\mu)$ the sequence $S_{n}(f) / a_{n}(T)$ converges weakly to a random variable $Y_{\alpha} \int_{X} f d \mu$ where

$$
E\left(\exp \left(z Y_{\alpha}\right)\right)=\sum_{n=0}^{\infty} \frac{\Gamma(1+\alpha)^{n} z^{n}}{\Gamma(1+\alpha n)}
$$

for $z \in \mathbb{C}$ when $0<\alpha \leq 1$ and for $|z|<1$ when $\alpha=0$.

Here, "weak convergence" means that for any bounded continuous function $g:[0, \infty] \rightarrow \mathbb{R}, f \in L_{+}^{1}, q \in \mathscr{P}(X), q \ll m$, we have

$$
\int_{X} g\left(\frac{1}{a_{n}(T)} S_{n}(f)\right) d q \rightarrow E\left(g\left(Y_{\alpha} \mu(f)\right)\right)
$$

as $n \rightarrow \infty$, where $\mu(f)=\int_{X} f d \mu$. For $\alpha=0$ the variable $Y_{0}$ is clearly exponential and for $\alpha=1, Y_{1}=1$.

The almost sure behaviour of $S_{n}(f)$ for nonnegative integrable functions $f$ has been obtained in [4-7]. We repeat here the main results for completeness. Define for $0<\alpha<1$

$$
K_{\alpha}=\frac{\Gamma(1+\alpha)}{\alpha^{\alpha}(1-\alpha)^{1-\alpha}} .
$$

The stochastic process $\left\{f \circ S^{k}\right\}_{k \geq 1}$, where $(\Omega, \mathscr{F}, P, S)$ is a measure preserving transformation, is called continued fraction mixing if

$$
\exists c \in \mathbb{R}_{+} \ni P\left(A \cap S^{-n} B\right) \leq c P(A) P(B) \quad \forall n \in \mathbb{N}, A \in \sigma\left\{f \circ S^{k}\right\}_{k=0}^{n-1}, B \in \mathscr{F}
$$
and,

such that

$$
\exists n_{1} \in \mathbb{N}, \quad\left\{\varepsilon_{n}\right\}_{n \geq n_{1}}, \quad \varepsilon_{n} \rightarrow 0 \text { as } n \rightarrow \infty
$$

$$
\begin{gathered}
\forall n \in \mathbb{N}, A \in \sigma\left\{f \circ S^{k}\right\}_{k=0}^{n-1}, B \in \mathscr{F}, j \geq n_{1}: \\
\left(1-\varepsilon_{j}\right) P(A) P(B) \leq P\left(A \cap S^{-(n+j)} B\right) \leq\left(1+\varepsilon_{j}\right) P(A) P(B) .
\end{gathered}
$$

The $\varepsilon_{n}\left(n \geq n_{1}\right)$ are known as the mixing coefficients.

The next four results are valid under the assumption that there is a DarlingKac set $A$ for $T$ whose return time process $\left\{\varphi \circ T_{A}^{n}\right\}_{n \geq 1}$ is continued fraction mixing on $A$. 
Theorem [4, Theorem 4]. Assume that the return sequence of $(X, \mathscr{F}, \mu, T)$ is regularly varying with index $\alpha \in(0,1)$ and satisfies

$$
a_{n}(T) \sim n^{\alpha} h(n) .
$$

Let $\phi(n) \uparrow$ and $\phi(n) / n \downarrow$ as $n \uparrow \infty$. Then:

If

$$
\sum_{n=1}^{\infty} \frac{1}{n} \exp [-\beta \phi(n)]<\infty
$$

for all $\beta>1$ then

$$
\limsup _{n \rightarrow \infty} \frac{1}{n^{\alpha} h(n / \phi(n)) \phi(n)^{1-\alpha}} S_{n}(f) \leq K_{\alpha} \int_{X} f d \mu
$$

a.e. for every $f \in L_{+}^{1}(\mu)$.

If

$$
\sum_{n=1}^{\infty} \frac{1}{n} \exp [-\beta \phi(n)]=\infty
$$

for all $\beta<1$ then

$$
\limsup _{n \rightarrow \infty} \frac{1}{n^{\alpha} h(n / \phi(n)) \phi(n)^{1-\alpha}} S_{n}(f) \geq K_{\alpha} \int_{X} f d \mu
$$

a.e. for every $f \in L_{+}^{1}(\mu)$, and

$$
\limsup _{n \rightarrow \infty} \frac{1}{n^{\alpha} h(n / \log \log (n))[\log \log (n)]^{1-\alpha}} S_{n}(f)=K_{\alpha} \int_{X} f d \mu
$$

a.e. for every $f \in L_{+}^{1}(\mu)$.

Proposition [5, Proposition 1]. Assume that the return sequence of $(X, \mathscr{F}, \mu, T)$ is regularly varying with index 1 and satisfies

$$
a_{n}(T) \sim n h(n) .
$$

Then there exists a constant $K_{T} \in(0,1]$ such that

$$
\limsup _{n \rightarrow \infty} \frac{1}{n h(n / \log \log (n))} S_{n}(f)=K_{T} \int_{X} f d \mu
$$

a.e. for every $f \in L_{+}^{1}(\mu)$.

Moreover, if $h(n / \log \log (n)) \sim h(n)$ as $n \rightarrow \infty$, then

$$
\limsup _{n \rightarrow \infty} \frac{1}{n h(n)} S_{n}(f)=\int_{X} f d \mu
$$

a.e. for every $f \in L_{+}^{1}(\mu)$.

Theorem [6, Theorem 1]. Assume that the return sequence of $(X, \mathscr{F}, \mu, T)$ is regularly varying with index $\alpha \in(0,1)$ and satisfies

$$
a_{n}(T) \sim n^{\alpha} h(n) .
$$

Then the random functions

$$
t \rightarrow \frac{1}{n^{\alpha} h(n / \log \log n)(\log \log n)^{1-\alpha}} S_{[n t]}(f) \quad\left(t \in \mathbb{R}_{+}\right)
$$


form a relatively compact set in the Skorokhod space $D\left(\mathbb{R}_{+}\right)$and its set of accumulation points is

$\left\{x: \mathbb{R}_{+} \rightarrow \mathbb{R}_{+}: x\right.$ absolutely continuous, increasing, and $\left.\left\|x^{\prime}(t)\right\|_{L^{1 /(1-\alpha)\left(\mathbb{R}_{+}\right)}} \leq K_{\alpha}\right\}$.

Theorem [7, Theorem 2]. Assume that $T$ has a Darling-Kac set whose return time process is continued fraction mixing with rates $\varepsilon_{n}=O\left(1 /(\log n)^{\delta}\right)(\delta>0)$, and

$$
a_{n}(T)=n^{\alpha} h(n)
$$

is regularly varying with index $\alpha \in(0,1]$ or $\alpha=0$ and with $h(n) \sim$ $\exp \left(\int_{1}^{t} \frac{\eta(t)}{t} d t\right)$, where $\eta$ is monotonic, $|\eta(t)| \downarrow 0$ as $t \uparrow \infty$, and $|\eta(t)| / \log t \rightarrow \infty$ as $t \rightarrow \infty$. Then for any function $f \in L^{1}(\mu)$

$$
\lim _{N \rightarrow \infty} \frac{1}{\log N} \sum_{n=1}^{N} \frac{1}{n a_{n}(T)} S_{n}(f)=\int_{X} f d \mu \quad \text { a.e. }
$$

\section{CONSERVATIVITY AND ERGODICITY OF MARKOV FIBRED SYSTEMS}

We consider various measurable and nonsingular transformations of the nonatomic probability space $(X, \mathscr{F}, m)$.

In this paper, a (Markov) fibred system on $X$ will mean a pair $(T, \mathscr{R})$, where $T: X \rightarrow X$ is such a transformation, and $\mathscr{R} \subset \mathscr{F}$ is a countable partition of $X$, generating $\mathscr{F}$, satisfying the following conditions.

\section{The Markov property.}

$$
T B=\bigcup_{b \in \mathscr{R}, b \cap T B \neq \varnothing} b \quad \forall B \in \mathscr{R}
$$

This version of the Markov property is topological (i.e. set theoretic). It is also possible to work with a measure theoretic version:

$$
T B=\bigcup_{b \in \mathscr{R}, m(b \cap T B)>0} b \bmod m \quad \forall B \in \mathscr{R}
$$

Local invertibility. For every $B \in \mathscr{R}, m(B)>0$ and $T$ is nonsingular, invertible on $B$.

For $k \geq 1$, let

$$
\mathscr{R}_{0}^{k-1}=\bigvee_{j=0}^{k-1} T^{-j} \mathscr{R}
$$

and for $0 \leq k<l<\infty$, let $\mathscr{F}_{k}^{l}$ denote the $\sigma$-algebra generated by $\bigcup_{j=k}^{l-1} T^{-j} \mathscr{R}$.

For $\mathbf{b}=\left(b_{1}, \ldots, b_{n}\right) \in \mathscr{R}^{n}$ set

$$
\text { [b] }=\bigcap_{j=1}^{n} T^{-j+1} b_{j} \in \mathscr{R}_{0}^{n-1} .
$$

Remark. If $[\mathbf{b}]=\left[b_{1}, \ldots, b_{n}\right] \in \mathscr{R}_{0}^{n-1}$, then it follows from local invertibility and the Markov property that

$$
m([\mathbf{b}])>0 \Leftrightarrow m\left(\left[b_{k}, b_{k+1}\right]\right)>0 \quad(1 \leq k \leq n-1)
$$

and, in this case, $T^{n}:[\mathbf{b}] \rightarrow T b_{n}$ is nonsingular and invertible on [b] . 
It follows that if $X \subseteq \mathscr{R}^{\mathbb{N}}$ and $X_{m}$ is the closed support of $m$ with respect to the product discrete topology on $\mathscr{R}^{\mathbb{N}}$, with $T: X_{m} \rightarrow X_{m}$ the shift, then $\left(X_{m}, T\right)$ is a topological Markov shift.

Accordingly, a fibred system $(T, \mathscr{R})$ is called irreducible if

$$
\forall B, B^{\prime} \in \mathscr{R}, \exists n \in \mathbb{N} \ni m\left(B \cap T^{-n} B^{\prime}\right)>0,
$$

and is called aperiodic if

$$
\forall B, B^{\prime} \in \mathscr{R}, \exists n \in \mathbb{N} \ni m\left(B \cap T^{-\nu} B^{\prime}\right)>0 \quad \forall \nu \geq n .
$$

Clearly,

$$
T \text { conservative and ergodic } \Rightarrow(T, \mathscr{R}) \text { irreducible, }
$$

and

$$
T \text { mixing with finite invariant measure } \Rightarrow(T, \mathscr{R}) \text { aperiodic. }
$$

Indeed the irreducibility (aperiodicity) of $(T, \mathscr{R})$ is equivalent to the topological ergodicity (mixing) of the underlying topological Markov shift.

Let $(T, \mathscr{R})$ be a fibred system on $X$. A subclass is a subset $\varnothing \neq \mathscr{R}^{\prime} \subseteq \mathscr{R}$ such that

$$
T b \subset \bigcup_{B \in \mathscr{R}^{\prime}} B \quad \forall b \in \mathscr{R}^{\prime} .
$$

Let $\mathscr{R}^{\prime} \subset \mathscr{R}$ be a subclass. Define $X^{\prime}:=\bigcup_{b \in \mathscr{R}^{\prime}} b$; then clearly $T X^{\prime} \subseteq X^{\prime} \subseteq$ $T^{-1} X^{\prime}$. In case $T X^{\prime}=X^{\prime},\left(T, \mathscr{R}^{\prime}\right)$ is called a subsystem (sometimes denoted by $X^{\prime}$ ).

If $X^{\prime}$ is a subsystem of $(T, \mathscr{R})$, then $T X^{\prime}=X^{\prime} \subseteq T^{-1} X^{\prime}$, and $X^{\prime}$ is a union of elements of $\{T b: b \in \mathscr{R}\}$. Clearly, a union of subsystems is itself a subsystem, and a fibred system having no proper subclasses is irreducible.

If $|\{T b: b \in \mathscr{R}\}|<\infty$, then any disjoint family of subclasses is finite, and each subclass has a further subclass which is a subsystem. Any subsystem $\left(T, \mathscr{R}^{\prime}\right)$ also has the property that $\left|\left\{T b: b \in \mathscr{R}^{\prime}\right\}\right|<\infty$. Thus every subsystem has an irreducible subsystem.

If, in addition, $(T, \mathscr{R})$ is not irreducible, then it is not hard to see that for any $b \in \mathscr{R}$, there exists $n \in \mathbb{N}$ such that $T^{n} b$ is contained in a proper subsystem. It follows, that if $X_{0}$ is the union of all the irreducible subsystems, then

$$
\bigcup_{n=1}^{\infty} T^{-n} X_{0}=X \quad \bmod m .
$$

Set, for $n \in \mathbb{N}$, and $B \in \mathscr{R}_{0}^{n-1}$,

$$
\Delta(B)=\Delta_{n}(B)=\left.\frac{d m \circ T^{n}}{d m}\right|_{B} .
$$

By local invertibility, $\Delta(B)>0$ a.e. on $B$.

Let

$$
\begin{gathered}
\widetilde{\mathscr{R}}=\bigcup_{n \in \mathbb{N}} \mathscr{R}_{0}^{n-1}, \\
\widetilde{\mathscr{R}}_{+}=\{[\mathbf{b}] \in \widetilde{\mathscr{R}}: m([\mathbf{b}])>0\},
\end{gathered}
$$


and for $C \in \mathbb{R}_{+}$set

$$
\mathscr{G}(C, T)=\left\{B \in \widetilde{\mathscr{R}}_{+}: \frac{\Delta(B)(x)}{\Delta(B)(y)} \leq C \text { for } m \times m \text { a.e. }(x, y) \in B \times B\right\} .
$$

It follows from local invertibility that if $[\mathbf{b}] \in \mathscr{R}_{0}^{n-1} \cap \mathscr{G}(C, T)$, then for $m$-a.e. $x \in[\mathbf{b}]$,

$$
m\left(T b_{n}\right)=\int_{[\mathbf{b}]} \Delta([\mathbf{b}]) d m=M m([\mathbf{b}]) \Delta([\mathbf{b}])(x),
$$

where $M \in\left[C^{-1}, C\right]$.

Therefore, if [b] $\in \mathscr{R}_{0}^{n-1} \cap \mathscr{G}(C, T),[\boldsymbol{\beta}] \in \mathscr{R}_{0}^{\nu-1} \cap \mathscr{G}(C, T)$, and also $[\mathbf{b}, \boldsymbol{\beta}] \in \mathscr{G}(C, T)$, then,

$$
\frac{1}{C^{3}} \leq \frac{m\left(T b_{n}\right) m([\mathbf{b}, \boldsymbol{\beta}])}{m([\mathbf{b}]) m([\boldsymbol{\beta}])} \leq C^{3}
$$

or, equivalently,

$$
C^{-3} m\left([\boldsymbol{\beta}] \mid T b_{n}\right) \leq m\left(T^{-n}[\boldsymbol{\beta}] \mid[\mathbf{b}]\right) \leq C^{3} m\left([\boldsymbol{\beta}] \mid T b_{n}\right),
$$

where the latter inequality is also valid for $[\mathbf{b}, \boldsymbol{\beta}]=\varnothing$. The formula $(\nabla)$ states that the metric distortions of powers of $T$ are uniformly bounded. This phenomenon will sometimes be referred to as the bounded (metric) distortion.

Example 1 (Markov shift). Let $S$ be a countable set, and let $P$ be a stochastic matrix on $S$. Set

$$
X=\left\{\mathbf{x}=\left(x_{1}, x_{2}, \ldots\right) \in S^{\mathbb{N}}: p_{x_{k}, x_{k+1}}>0 \forall k \geq 1\right\},
$$

$T: X \rightarrow X$ the shift, $\mathscr{R}=\{[s]\}_{s \in S}$, and for $q \in \mathscr{P}(S), q_{s}>0 \forall s$, define $m \in \mathscr{P}(X)$ by

$$
m\left(\left[s_{1}, \ldots, s_{n}\right]\right)=q_{s_{1}} \prod_{k=1}^{n-1} p_{s_{k}, s_{k+1}} .
$$

It follows that $(T, \mathscr{R})$ is a fibred system, and that for $n \geq 1$, and [s] = $\left[s_{1}, \ldots, s_{n}\right] \in \mathscr{R}_{0}^{n-1}$,

$$
\Delta_{n}([\mathbf{s}])=\frac{1}{m([\mathbf{s}])} \frac{q_{t}}{p_{s_{n}, t}} \quad \text { a.e. on }[\mathbf{s}, t] \quad(t \in S) .
$$

Hence,

$$
\begin{aligned}
{\left[s_{1}, \ldots, s_{n}\right] \in \mathscr{G}(C, T) } & \Leftrightarrow \frac{p_{s_{n}, t} q_{t^{\prime}}}{p_{s_{n}, t^{\prime}} q_{t}} \in\left[C^{-1}, C\right]\left(\text { whenever } p_{s_{n}, t}, p_{s_{n}, t^{\prime}}>0\right) \\
& \Leftrightarrow\left[s_{n}\right] \in \mathscr{G}(C, T) .
\end{aligned}
$$

For $s \in S$, if $1 / 2 \leq q_{t} / p_{s, t} \leq 2$ whenever $p_{s, t}>0$ then $[s] \in \mathscr{R}(4, T)$. Clearly, for $s \in S$, there is $q \in \mathscr{P}(S)$ with this property.

Lemma 2.1. Suppose $(T, \mathscr{R})$ is a fibred system such that $\mathscr{G}(C, T)=\widetilde{\mathscr{R}}_{+}$, and $\inf \{m(B): B \in T \mathscr{R}\}>0$. Then there is a $T$-invariant probability $q \ll m$, with $\frac{d q}{d m} \in L^{\infty}$ and such that $\left[\frac{d q}{d m}>0\right]$ is a subsystem. In case $|T \mathscr{R}|<\infty$,

$$
\log \frac{d q}{d m} \in L^{\infty}\left(\left.m\right|_{X^{\prime}}\right)
$$


Proof. Since $\mathscr{G}(C, T)=\widetilde{R}$, and $\mathscr{R}$ generates $\mathscr{F}$ under $T$, it follows from bounded distortion $(\mathcal{Q})$ that $\forall n \geq 1$, [b] $\in \mathscr{R}_{0}^{n-1}$, and $A \in \mathscr{F}$,

$$
C^{-3} m\left(A \mid T b_{n}\right) \leq m\left(T^{-n} A \mid[\mathbf{b}]\right) \leq C^{3} m\left(A \mid T b_{n}\right) .
$$

Set $\beta=\{T b: b \in \mathscr{R}\}$, and suppose $m(B) \geq \varepsilon>0 \forall B \in \beta$. For $A \in \mathscr{F}$ and $n \geq 1$, we have

$$
\begin{aligned}
m\left(T^{-n} A\right) & =\sum_{\mathbf{b} \in \mathscr{R}^{n}} m\left(T^{-n} A \mid[\mathbf{b}]\right) m([\mathbf{b}]) \\
& \leq C^{3} \sum_{\mathbf{b} \in \mathscr{R}^{n}} m\left(A \mid T b_{n}\right) m([\mathbf{b}]) \leq \frac{C^{3}}{\varepsilon} m(A) .
\end{aligned}
$$

Hence

$$
\sup _{n \geq 1}\left\|\frac{1}{n} \sum_{k=0}^{n-1} \frac{d m \circ T^{-k}}{d m}\right\|_{\infty}<\infty,
$$

and the sequence $\left\{\frac{1}{n} \sum_{k=0}^{n-1} \frac{d m o T^{-k}}{d m}\right\}_{n \geq 1}$ is weak ${ }^{*}$-compact in $L^{\infty}(m)$. Any weak limit is the density of a $T$-invariant probability on $X$. In other words, there is a probability $q \ll m$ on $X$ such that $q \circ T^{-1}=q$ and $\frac{d q}{d m} \in L^{\infty}(m)$.

Set

$$
\nu_{n}(A)=\sum_{B \in \beta} m(A \mid B) \sum_{a \in \mathscr{R}, T a=B} m\left(T^{-(n-1)} a\right) \quad(n \in \mathbb{N}, A \in \mathscr{F}) .
$$

then, by the above,

$$
\frac{m\left(T^{-n} A\right)}{\nu_{n}(A)} \in\left[C^{-3}, C^{3}\right] \quad(n \in \mathbb{N}, A \in \mathscr{F})
$$

and

$$
\frac{d \nu_{n}}{d m}=\sum_{B \in \beta} \frac{\sum_{a \in \mathscr{R}, T a=B} m\left(T^{-(n-1)} a\right)}{m(B)} 1_{B} .
$$

By construction of $q$, there is a subsequence $n_{k} \rightarrow \infty$ such that

$$
\lim _{k \rightarrow \infty} \frac{1}{n_{k}} \sum_{j=0}^{n_{k}-1} m\left(T^{-j} A\right)=q(A) \quad \forall A \in \mathscr{F},
$$

whence

$$
\frac{d \lambda_{n_{k}}}{d m} \stackrel{L^{\infty}(m)}{\rightarrow} \sum_{B \in \beta} \frac{\sum_{a \in \mathscr{R}, T a=B} q^{(a)}}{m(B)} 1_{B}=\frac{d \nu}{d m},
$$

where

$$
\nu(A)=\sum_{B \in \beta} \sum_{a \in \mathscr{R}, T a=B} q^{(a)} m(A \mid B) \quad \forall A \in \mathscr{F}
$$

and

$$
\lambda_{n}=\frac{1}{n} \sum_{j=0}^{n-1} \nu_{j}
$$

It follows that

$$
\frac{d q}{d m}=C^{ \pm 3} \sum_{B \in \beta} \frac{1}{m(B)} \sum_{a \in \mathscr{R}, T a=B} q^{(a)} 1_{B} .
$$


Let $\mathscr{R}^{\prime}=\{b \in \mathscr{R}: q(b)>0\}$ and $X^{\prime}=\bigcup_{b \in \mathscr{R}^{\prime}} b$. Evidently $\left[\frac{d q}{d m}>0\right]=$ $X^{\prime}$. Next we claim that $\left(T, \mathscr{R}^{\prime}\right)$ is a subsystem. To see this, note that, for $b \in \mathscr{R}^{\prime},\left[\frac{d q}{d m}>0\right]$ on $T b$, hence $T b \subseteq \bigcup_{a \in \mathscr{R}^{\prime}} a$, and $\mathscr{R}^{\prime}$ is a subclass. As $T^{-1}\left(X^{\prime} \backslash T X^{\prime}\right) \subseteq X \backslash X^{\prime}$, it follows that $q\left(X^{\prime} \backslash T X^{\prime}\right)=0$, whence, by the above, $T X^{\prime}=X^{\prime}$ and $X^{\prime}$ is a subsystem. In case $|T \mathscr{R}|<\infty$, the nonzero values of $\frac{d \nu}{d m}$ are uniformly bounded below. Hence, the nonzero values of $\frac{d q}{d m}$ are also uniformly bounded below, whence $\log \frac{d q}{d m} \in L^{\infty}\left(\left.m\right|_{X^{\prime}}\right)$. $\diamond$

Example 2. Let $P$ be a stochastic matrix defined on $S=\mathbb{Z}$ by $p_{n, n+1}=p$, and $p_{n, n-1}=1-p$, where $p \in(0,1)$.

Let $X \subset \mathbb{Z}^{\mathbb{N}}$ be defined as in Example 1, let $T: X \rightarrow X$ be the shift, and define $m \in \mathscr{P}(X)$ by

$$
m\left(\left[s_{1}, \ldots, s_{n}\right]\right)=q_{s_{1}} \prod_{k=1}^{n-1} p_{s_{k}, s_{k+1}},
$$

where $q_{s}=\frac{1}{2^{\mid s 3} 3}$.

If $\mathscr{R}=\{[s]\}_{s \in \mathbb{Z}}$, then $(T, \mathscr{R})$ is an irreducible fibred system, and $\mathscr{G}(C, T)$ $=\widetilde{\mathscr{R}}$ for $C=4 \frac{p \vee(1-p)}{p \wedge(1-p)}$.

However the conclusion of Lemma 2.1 does not hold since $T$ is totally dissipative for $p \neq 1 / 2$, and $T$ is conservative, ergodic, and with an infinite invariant measure $\mu \sim m$ for $p=1 / 2$.

Example 3. As in the example of $\S 1$, let $X=[0,1), m$ be Lebesgue measure, and $T: X \rightarrow X$ be defined by

$$
T x= \begin{cases}2 x, & x \in[0,1 / 4), \\ 2 x-1 / 2, & x \in[1 / 4,1 / 2), \\ 2 x-1, & x \in[1 / 2,1) .\end{cases}
$$

If $\mathscr{R}=\{[0,1 / 4),[1 / 4,1 / 2),[1 / 2,1)\}$, then $(T, \mathscr{R})$ is a fibred system, and $\mathscr{G}(1, T)=\widetilde{R}$, since $T^{\prime} \equiv 2$.

The conservative part of $T$ is $[0,1 / 2)$, and this is the subsystem of Lemma 2.1.

Lemma 2.2. Suppose $(T, \mathscr{R})$ is a fibred system such that $\mathscr{G}(C, T)=\widetilde{\mathscr{R}}_{+}$and $|\{T b: b \in \mathscr{R}\}|<\infty$, and let $X_{c} \in \mathscr{F}$ be the conservative part of $T$. Then

$$
\bigcup_{n=1}^{\infty} T^{-n} X_{c}=X \bmod m \text {. }
$$

Proof. By Lemma 2.1, if $X^{\prime}$ is an irreducible subsystem, then

$$
\exists q \in \mathscr{P}\left(X^{\prime}\right),\left.q \sim m\right|_{X^{\prime}}, q \circ T^{-1}=q,
$$

whence $X^{\prime} \subseteq X_{c}$. If $X_{0}$ is the union of the irreducible subsystems of $(T, \mathscr{R})$, then $X_{0} \subseteq X_{c}$. $\diamond$

As shown above,

$$
\bigcup_{n=1}^{\infty} T^{-n} X_{0}=X \quad \bmod m
$$


whence

$$
\bigcup_{n=1}^{\infty} T^{-n} X_{c}=X \bmod m . \diamond
$$

Schweiger property. The Schweiger property is $\exists C \in \mathbb{R}_{+}$, and a collection of sets $\mathscr{R}(C, T) \subseteq \mathscr{G}(C, T)$ such that

$$
[\mathbf{b}] \in \mathscr{R}(C, T),[\boldsymbol{\beta}] \in \widetilde{\mathscr{R}},[\boldsymbol{\beta}, \mathbf{b}] \neq \varnothing \Rightarrow[\boldsymbol{\beta}, \mathbf{b}] \in \mathscr{R}(C, T)
$$

and

$$
\bigcup_{B \in \mathscr{R}(C, T)} B=X \bmod m .
$$

It follows from this that $\mathscr{R}(C, T)$ generates $\mathscr{F}$.

Clearly, if $\mathscr{R}(C, T)$ is such a collection, then

$$
\mathscr{R}(C, T) \subseteq \overline{\mathscr{R}}(C, T),
$$

where

$$
\overline{\mathscr{R}}(C, T):=\{[\mathbf{b}] \in \mathscr{G}(C, T):[\boldsymbol{\beta}] \in \widetilde{\mathscr{R}},[\boldsymbol{\beta}, \mathbf{b}] \neq \varnothing \Rightarrow[\boldsymbol{\beta}, \mathbf{b}] \in \mathscr{G}(C, T)\}
$$

and so $T$ has the Schweiger property if and only if

$$
\bigcup_{B \in \bar{R}(C, T)} B=X \bmod m
$$

for some $C \geq 1$. It is not hard to show that if $T$ is conservative and ergodic, and $B \in \overline{\mathscr{R}}(C, T)$, then $T$ has the Schweiger property with respect to

$$
\mathscr{R}(C, T):=\left\{\left[b_{1}, \ldots, b_{n}\right] \cap T^{-n} B: n \geq 0,\left[b_{1}, \ldots, b_{n}\right] \in \mathscr{R}_{0}^{n-1}\right\} .
$$

If $(T, \mathscr{R})$ is an irreducible recurrent Markov shift, then it is well known that $(T, \mathscr{R})$ is conservative ergodic, and, by the discussion of Example $1,(T, \mathscr{R})$ has the Schweiger property if and only if $\mathscr{G}(C, T) \neq \varnothing$ for some $C \geq 1$. Indeed, it now follows that any irreducible, recurrent Markov shift has the Schweiger property with respect to some initial probability distribution.

Lemma 2.3. Suppose $(T, \mathscr{R})$ is a fibred system which has the Schweiger property with respect to $\mathscr{R}(C, T)$, and suppose $A \in \mathscr{R}(C, T)$. Then

$$
\begin{gathered}
\forall B \in \mathscr{F} \cap A, n \in \mathbb{N},[\mathbf{b}] \in \mathscr{R}_{0}^{n-1}, \exists M \in\left[C^{-6}, C^{6}\right] \\
\ni m\left([\mathbf{b}] \cap T^{-n} B\right)=M m\left([\mathbf{b}] \cap T^{-n} A\right) m(B \mid A) .
\end{gathered}
$$

Proof. Suppose $A=[\mathbf{a}]=\left[a_{1}, \ldots, a_{n}\right] \in \mathscr{R}(C, T)$, and fix $[\mathbf{c}] \in \mathscr{R}(C, T)$. For $k \geq 1$ and [b] $\in \mathscr{R}_{0}^{k-1}$, it follows from bounded distortion $(\nabla)$ that

$$
\begin{aligned}
m([\mathbf{b}, \mathbf{a}, \mathbf{c}]) & =\frac{M}{m\left(T a_{n}\right)} m([\mathbf{b}, \mathbf{a}]) m([\mathbf{c}]) \quad(\text { since }[\mathbf{b}, \mathbf{c}] \in \mathscr{G}(C, T)) \\
& =M M^{\prime} \frac{m([\mathbf{b}, \mathbf{a}])}{m([\mathbf{a}])} m([\mathbf{a}, \mathbf{c}]) \quad(\text { since }[\mathbf{a}, \mathbf{c}],[\mathbf{c}] \in \mathscr{G}(C, T)) \\
& =M M^{\prime} m([\mathbf{b}, \mathbf{a}]) m([\mathbf{a}, \mathbf{c}] \mid A)
\end{aligned}
$$

where $M, M^{\prime} \in\left[C^{-3}, C^{3}\right]$, and the result follows since $\mathscr{R}(C, T)$ generates $\mathscr{F} . \diamond$ 
Theorem 2.4. Suppose $(T, \mathscr{R})$ is a fibred system which has the Schweiger property with respect to $\mathscr{R}(C, T)$. Let $X_{c}$ be the conservative part of $T$, and suppose $A \in \mathscr{R}(C, T)$. Then

$$
\sum_{n=1}^{\infty} m\left(T^{-n} A\right)=\infty \Rightarrow A \subset X_{c} \bmod m
$$

and

$$
\sum_{n=1}^{\infty} m\left(T^{-n} A\right)<\infty \Rightarrow A \subset X \backslash X_{c} \bmod m .
$$

In particular, $X_{c}$ and $X_{d}$ are both unions of sets in $\mathscr{R}(C, T)$.

Proof. The second implication is clear. To prove the first, suppose $A \in$ $\mathscr{R}(C, T)$ and $m\left(A \backslash X_{c}\right)>0$. Then

$$
\exists B \in \mathscr{F} \cap A, \quad m(B)>0, \quad \ni \sum_{n=1}^{\infty} m\left(T^{-n} B\right)<\infty,
$$

whence, by Lemma 2.3 ,

$$
\sum_{n=1}^{\infty} m\left(T^{-n} A\right)<\infty \quad \diamond
$$

Theorem 2.5. Suppose $(T, \mathscr{R})$ is an irreducible fibred system which has the Schweiger property. Then $T$ is either conservative or totally dissipative; if $T$ is conservative, then $T$ is ergodic.

Proof. Assume that

$$
\bigcup_{B \in \mathscr{R}(C, T)} B=X \bmod m .
$$

It follows from Theorem 2.4 that

$$
X_{c}=\bigcup_{B \in \mathscr{R}(C, T) \cap X_{c}} B \bmod m .
$$

Therefore, it follows from irreducibility that $T$ is either conservative, or totally dissipative. Suppose $T$ is conservative. Since $\mathscr{R}(C, T)$ generates $\mathscr{F}$, it follows from bounded distortion $(\mathcal{O})$ that

$$
\frac{m\left(T^{-n} A \mid[\mathbf{b}]\right)}{m\left(A \mid T b_{n}\right)} \in\left[C^{-3}, C^{3}\right] \quad \forall n \in \mathbb{N},[\mathbf{b}] \in \mathscr{R}_{0}^{n-1} \cap \mathscr{R}(C, T), A \in \mathscr{F} .
$$

Now suppose that $A \in \mathscr{F}, T^{-1} A=A$, and $m(A)>0$. Then

$$
\frac{m(A \mid[\mathbf{b}])}{m\left(A \mid T b_{n}\right)} \in\left[C^{-3}, C^{3}\right] \quad \forall n \in \mathbb{N},[\mathbf{b}] \in \mathscr{R}_{0}^{n-1} \cap \mathscr{R}(C, T) .
$$

By the martingale convergence theorem, for $m$-a.e. $x \in X$,

$$
m\left(A \mid\left[b_{1}(x), \ldots, b_{n}(x)\right]\right) \rightarrow 1_{A}(x) \text { as } n \rightarrow \infty,
$$

where, for $n \geq 1, b_{n}(x)$ is defined by $T^{n-1} x \in b_{n}(x) \in \mathscr{R}$. 
By conservativity of $T$, if $[\mathbf{b}]=\left[b_{1}, \ldots, b_{n}\right] \in \mathscr{R}(C, T)$, then, for $m$-a.e. $x \in[\mathbf{b}], T^{k} x \in[\mathbf{b}]$ (that is, $\left.\left(b_{k+1}(x), \ldots, b_{k+n}(x)\right)=\mathbf{b}\right)$ for infinitely many $k$, hence

$$
\frac{1_{A}(x)}{m\left(A \mid T b_{n}\right)} \in\left[C^{-3}, C^{3}\right] .
$$

It follows that

$$
A=\bigcup_{B \in \mathscr{R}(C, T), m(A \cap B)>0} B \bmod m .
$$

Since $m(A)>0$,

$$
\exists B \in \mathscr{R}(C, T) \quad \ni B \subset A \bmod m .
$$

By irreducibility, if $B^{\prime} \in \mathscr{R}(C, T)$, then

$$
\exists k \geq 0 \ni m\left(B \cap T^{-k} B^{\prime}\right)>0,
$$

whence $B^{\prime} \subset A$. Thus $A=X \bmod m$, and $T$ is ergodic.

Suppose that $(T, \mathscr{R})$ has the Schweiger property, with respect to the collection $\mathscr{R}(C, T)$, and let $N_{C}: X \rightarrow \mathbb{N} \cup \infty$ be defined by

$$
N_{C}(x)=\inf \left\{n \in \mathbb{N}:\left[b_{1}(x), \ldots, b_{n}(x)\right] \in \mathscr{R}(C, T)\right\}
$$

where $\inf \varnothing:=\infty$. Then $N_{C}<\infty$ a.e.

Schweiger's jump transformation $T^{*}: X \rightarrow X$ is defined by

$$
T^{*}(x)=T^{N_{C}(x)}(x) .
$$

By the Schweiger property, $T^{*}(x)$ is defined for $m$-a.e. $x \in X$.

If $T: B \rightarrow X$ is onto $\forall B \in \mathscr{R}$, then $\left(X, \mathscr{F}, m, T^{*}\right)$ is a nonsingular transformation. In general, by the Markov property,

$$
\forall n \geq 1, \exists \tau_{n} \subseteq \mathscr{R} \quad \ni \quad T^{* n} X=\bigcup_{b \in \tau_{n}} b .
$$

Clearly, $T^{* n} X \supset T^{* n+1} X$, and $\tau_{n} \supset \tau_{n+1} \forall n \geq 1$, whence

$$
X^{*}:=\bigcap_{n=1}^{\infty} T^{* n} X=\bigcup_{b \in \tau} b \bmod m,
$$

where $\tau=\bigcap_{n \in \mathbb{N}} \tau_{n}$.

It is not hard to show that $\left(X^{*}, \mathscr{F},\left.m\right|_{X^{*}}, T^{*}\right)$ is a nonsingular transformation in case $m\left(X^{*}\right)>0$ (i.e., $\left.\tau \neq \varnothing\right)$.

Lemma 2.6. Suppose $(T, \mathscr{R})$ is a fibred system with the Schweiger property, and $\inf \{m(B): B \in T \mathscr{R}\}>0$. Then $m\left(X^{*}\right)>0$.

Proof. Let $\beta=\{T b: b \in \mathscr{R}\}$; then it follows from $\inf \{m(B): B \in T \mathscr{R}\}>0$ that

$$
\exists \mathscr{R}_{0} \subseteq \mathscr{R},\left|\mathscr{R}_{0}\right|<\infty \ni B \cap \mathscr{R}_{0} \neq \varnothing \quad \forall B \in \beta .
$$

Also,

$$
\forall n \geq 1, \quad \exists \varnothing \neq \mathscr{R}_{n} \subseteq \mathscr{R} \quad \ni \quad T^{* n} X=\bigcup_{B \in \mathscr{R}_{n}} B .
$$


Since, for every $n \geq 1, T^{* n} X$ is a union of sets in $\beta$, it follows that $1 \leq$ $\left|\mathscr{R}_{n} \cap \mathscr{R}_{0}\right|<\infty \quad \forall n \geq 1$, whence, since $\mathscr{R}_{n} \supset \mathscr{R}_{n+1}$,

$$
\bigcap_{n \in \mathbb{N}} \mathscr{R}_{n} \supset \bigcap_{n \in \mathbb{N}} \mathscr{R}_{n} \cap \mathscr{R}_{0} \neq \varnothing
$$

whence $m\left(X^{*}\right)>0$. $\diamond$

Note that $N_{C}$ is a stopping time with respect to $\mathscr{R}$ in the sense that $\left[N_{C}=\right.$ $n] \in \mathscr{F}_{0}^{n-1} \quad \forall n \in \mathbb{N}$.

Define the partition

$$
\mathscr{R}^{*}=\bigcup_{n=1}^{\infty} X^{*} \cap\left[N_{C}=n\right] \cap \mathscr{R}_{0}^{n-1} .
$$

It follows that $\left(T^{*}, \mathscr{R}^{*}\right)$ is a fibred system on $X^{*}$, and $\mathscr{G}\left(C, T^{*}\right)=\widetilde{\mathscr{R}}_{+}^{*}$. Also, by the Markov property,

$$
\left\{T^{*} b: b \in \mathscr{R}^{*}\right\} \subseteq\{T b: b \in \mathscr{R}\},
$$

whence

$$
|\{T b: b \in \mathscr{R}\}|<\infty \Rightarrow\left|\left\{T^{*} b: b \in \mathscr{R}^{*}\right\}\right|<\infty
$$

and

$$
\inf \{m(B): B \in T \mathscr{R}\}>0 \Rightarrow \inf \left\{m(B): B \in T^{*} \mathscr{R}^{*}\right\}>0 .
$$

Lemma 2.7. Suppose $(T, \mathscr{R})$ is an irreducible fibred system which has the Schweiger property, and suppose $[\mathrm{b}] \in \widetilde{\mathscr{R}}$. Then

$$
\forall A \in \mathscr{F}, m(A)>0, \exists n \in \mathbb{N} \ni m\left([\mathbf{b}] \cap T^{-n} A\right)>0 .
$$

Proof. Assume

$$
\bigcup_{B \in \mathscr{R}(C, T)} B=X \bmod m .
$$

Fix [b] $\in \widetilde{\mathscr{R}}$ and suppose [a] $=\left[a_{1}, \ldots, a_{n}\right] \in \mathscr{R}(C, T)$. By irreducibility, there is a $\left[\mathbf{b}^{\prime}\right] \subset[\mathbf{b}]$ such that $m\left(\left[\mathbf{b}^{\prime}, \mathbf{a}\right]\right)>0$. Clearly, $\left[\mathbf{b}^{\prime}, \mathbf{a}\right] \in \mathscr{R}(C, T)$, and it follows from bounded distortion $(Q)$ that if $[\mathrm{c}] \in \mathscr{R}(C, T)$, then

$$
\begin{aligned}
m\left(\left[\mathbf{b}^{\prime}, \mathbf{a}, \mathbf{c}\right]\right) & \geq \frac{1}{C^{3} m\left(T a_{n}\right)} m\left(\left[\mathbf{b}^{\prime}, \mathbf{a}\right]\right) m([\mathbf{c}]) \\
& \geq \frac{m\left(\left[\mathbf{b}^{\prime}, \mathbf{a}\right]\right)}{C^{6} m([\mathbf{a}])} m([\mathbf{a}, \mathbf{c}]):=\eta m([\mathbf{a}, \mathbf{c}]),
\end{aligned}
$$

whence, for $A \in \mathscr{F} \cap[\mathbf{a}]$,

$$
m\left([\mathbf{b}] \cap T^{-k} A\right) \geq \eta m(A) .
$$

The result follows as

$$
\forall A \in \mathscr{F}, m(A)>0, \exists[\mathbf{a}] \in \mathscr{R}(C, T) \quad \ni \quad m([\mathbf{a}] \cap A)>0 . \diamond
$$

Theorem 2.8. Suppose $(T, \mathscr{R})$ is an irreducible fibred system with the Schweiger property, and $\inf \{m(B): B \in T \mathscr{R}\}>0$. Then $T$ is conservative and ergodic.

Proof. Suppose $T$ has the Schweiger property with respect to $\mathscr{R}(C, T)$. By Lemma 2.6, $m\left(X^{*}\right)>0$, whence $\left(T^{*}, \mathscr{R}^{*}\right)$ is a fibred system such that 
$\mathscr{G}\left(C, T^{*}\right)=\widetilde{\mathscr{R}}^{*}$ and $\inf \left\{m(B): B \in T^{*} \mathscr{R}^{*}\right\}>0$. By Lemma 2.1 , the conservative part of $T^{*}, X_{c}$, has positive measure, and, by Theorem 2.4 , is a union of sets in $\mathscr{R}\left(C, T^{*}\right) \subseteq \widetilde{R}$. If $W \in \mathscr{F}$ and $m(W)>0$ is a wandering set for $T$, then, by Lemma 2.7, for some $n \in \mathbb{N}, m\left(X_{c} \cap T^{-n} W\right)>0$. The set $W_{n}=X_{c} \cap T^{-n} W$ is also a wandering set for $T$. Since $W_{n} \subset X_{c} \subset X^{*}$,

$$
\bigcup_{\nu=1}^{\infty} T^{*-\nu} W_{n} \subseteq \bigcup_{\nu=1}^{\infty} T^{-\nu} W_{n} \subseteq W_{n}^{c},
$$

whence, by the conservativity of $T^{*}$ on $X_{c}, m\left(W_{n}\right)=0$. This contradiction establishes the conservativity of $T$. By Theorem 2.5, T is ergodic. $\diamond$

Examples: Markov maps of the interval. A map $T: I \rightarrow I$ ( $I$ a bounded interval) is called Markov if there is a partition $\mathscr{A}$ of $I$ into intervals such that

1. $\forall A \in \mathscr{A},\left.T\right|_{A}$ is strictly monotonic and extends to a $\mathrm{C}^{2}$-function on $\bar{A}$.

2. $(T, \mathscr{A})$ is a fibred system.

It can be shown (using the methods of [35]) that if in addition

(a) for each $A \in \mathscr{A}, \bar{A}$ contains at most one fixed point,

(b) the set $\Lambda$ of indifferent fixed points is finite,

(c) $\forall \varepsilon>0 \exists \rho(\varepsilon)>1 \ni T^{\prime} x \geq \rho(\varepsilon) \forall x \in A \backslash B(\Lambda, \varepsilon)$,

(d) $\exists \eta>0 \ni T^{\prime} \downarrow$ on $(y-\eta, y) \cap A_{y}$ and $T^{\prime} \uparrow$ on $(y, y+\eta) \cap A_{y} \forall y \in \Lambda$ (here for $y \in \Lambda, A_{y} \in \mathscr{A}$ and $y \in \bar{A}_{y}$ ), and

(e) $\left|T^{\prime \prime}\right| /\left(T^{\prime}\right)^{2}$ is uniformly bounded on $I$, then $(T, \mathscr{A})$ has the Schweiger property with respect to

$$
\left\{\left[a_{1}, \ldots, a_{n}\right] \text { : either } \omega_{a_{n}} \notin \Lambda \text { or } a_{n-1} \neq a_{n}\right\} \text {. }
$$

\section{INVARIANT MEASURES, DARLING-KAC SETS, AND EXACTNESS FOR MARKOV FIBRED SYSTEMS}

Theorem 3.1. Suppose that $(T, \mathscr{R})$ is an irreducible fibred system having the Schweiger property with respect to $\mathscr{R}(C, T)$, and $T$ is conservative. Then $T$ is ergodic and there is a $\sigma$-finite, $T$-invariant measure $\mu \sim m$ such that

$$
\log \frac{d \mu}{d m} \in L^{\infty}(B) \quad \forall B \in \mathscr{R}(C, T) .
$$

Moreover, any $A \in \mathscr{R}(C, T)$ is a Darling-Kac set for $T$ whose return time process is continued fraction mixing.

Definition (see $\S 1)$. Let $(X, \mathscr{F}, m, T)$ be a nonsingular transformation, $q \sim$ $m$ a $T$-invariant probability, and $\mathscr{R} \subset \mathscr{F}$ a partition of $X$.

The system $(T, \mathscr{R})$ is called continued fraction mixing with respect to the $T$-invariant probability $q \sim m$, if

$$
\exists c \in \mathbb{R}_{+} \ni q\left([\mathbf{b}] \cap T^{-n} B\right) \leq c q([\mathbf{b}]) q(B) \quad \forall n \in \mathbb{N},[\mathbf{b}] \in \mathscr{R}_{0}^{n-1}, B \in \mathscr{F},
$$

and

$$
\exists n_{1} \in \mathbb{N}, \quad\left\{\varepsilon_{n}\right\}_{n \geq n_{1}}, \quad \varepsilon_{n} \rightarrow 0 \quad \text { as } n \rightarrow \infty
$$


such that

$$
\begin{gathered}
\forall k \in \mathbb{N}, A \in \mathscr{R}_{0}^{k-1}, B \in \mathscr{F}, n \geq n_{1}: \\
\left(1-\varepsilon_{n}\right) q(A) q(B) \leq q\left(A \cap T^{-(k+n)} B\right) \leq\left(1+\varepsilon_{n}\right) q(A) q(B) .
\end{gathered}
$$

Induced systems. Suppose $(T, \mathscr{R})$ is a fibred system and $T$ is conservative. For $A \in \mathscr{F}$, let $\varphi: A \rightarrow \mathbb{N}$ be the first return time to $A$ under $T$, namely, $\varphi(x)=\min \left\{n \in \mathbb{N}: T^{n} x \in A\right\}$. (By conservativity, $\varphi<\infty$ a.e. on $A$.) The induced transformation on $A$ is defined by $T_{A} x=T^{\varphi(x)} x$.

If $k \in \mathbb{N}$ and $A \in \mathscr{F}_{0}^{k-1}$ then $[\varphi=n] \in \mathscr{F}_{0}^{n+k-1} \forall n \in \mathbb{N}$. In this case, define the partition

$$
\mathscr{R}_{A}=\bigcup_{n=1}^{\infty}[\varphi=n] \cap \mathscr{R}_{0}^{n+k-1} .
$$

It follows that $\left(T_{A}, \mathscr{R}_{A}\right)$ is a fibred system on $A$, the induced fibred system on A.

Proof of Theorem 3.1. The ergodicity of $T$ follows from Theorem 2.5.

To show the existence of an invariant measure with the required properties, it suffices (see $\S 1$ ) to show that for every $A \in \mathscr{R}(C, T)$, there is a $T_{A}$-invariant measure $\left.\mu \sim m\right|_{A}$ such that $\log \frac{d \mu}{d m} \in L^{\infty}(A)$.

This is indeed the case by Lemma 2.1 , as $T_{A}(b)=A \forall b \in \mathscr{R}_{A}$, and $\mathscr{G}\left(C, T_{A}\right)=\left(\widetilde{\mathscr{R}}_{A}\right)_{+}$as $A \in \mathscr{R}(C, T)$.

To complete the proof of the theorem, it suffices to show that every $A \in$ $\mathscr{R}(C, T)$ is a Darling-Kac set whose return time process is continued fraction mixing.

For $A \in \mathscr{R}(C, T)$,

$$
\mathscr{G}(C, T)=\left(\widetilde{\mathscr{R}}_{A}\right)_{+} \quad \text { and } \quad T_{A} b=A \quad \forall b \in \mathscr{R}_{A},
$$

and so, by bounded distortion $(\nabla)$, there exists a $D>0$ such that

$$
\frac{1}{D} q(b) q(B) \leq q\left(b \cap T_{A}^{-n} B\right) \leq D q(b) q(B) \quad \forall n \geq 1, b \in \mathscr{R}_{0}^{n-1}, B \in \mathscr{F} \cap A .
$$

It follows from [9] that $\left(T_{A}, \mathscr{R}_{A}\right)$ is continued fraction mixing, whence, evidently, $A$ 's return time process is continued fraction mixing, and, by the main lemma in [3], $A$ is a Darling-Kac set for $T . \diamond$

The following theorem generalises, and was inspired by [36, Theorem 1].

Theorem 3.2. Suppose that $(T, \mathscr{R})$ is an aperiodic fibred system having the Schweiger property with respect to $\mathscr{R}(C, T)$, and $T$ is conservative. Then, $T$ is exact.

Proof. By Theorem 2.5, $T$ is ergodic. Suppose

$$
A \in \bigcap_{n=1}^{\infty} T^{-n \mathscr{F}}, \quad m(A)>0
$$

then

$$
\forall n \geq 0, \quad \exists A_{n} \in \mathscr{F} \quad \ni \quad A=T^{-n} A_{n} .
$$

It follows that $A_{n}=T^{n} A$, whence $A=T^{-n} T^{n} A$, and

$$
T^{-k} A_{n+k}=T^{-k} T^{n+k} A=T^{n}\left(T^{-(k+n)} T^{(k+n)} A\right)=T^{n} A=A_{n} .
$$


Since $m(A)>0$,

$$
\exists[\boldsymbol{\beta}]=\left[\beta_{1}, \ldots, \beta_{\nu}\right] \in \mathscr{R}(C, T) \quad \ni \quad m(A \cap[\boldsymbol{\beta}])>0 .
$$

Set

$$
\varphi(x)=\min \left\{n \in \mathbb{N}: T^{n} x \in[\boldsymbol{\beta}]\right\} .
$$

Then $\varphi<\infty$ a.e. as $T$ is conservative and ergodic. Define $T^{*}: X \rightarrow[\beta]$ by

$$
T^{*} x=T^{\varphi(x)} x
$$

Then $\left.T^{*}\right|_{[\beta]}=T_{[\beta]}$, the induced transformation which is conservative and ergodic on $[\boldsymbol{\beta}]$. For $k \geq 1$, let

$$
\varphi_{k}(x)=\sum_{j=0}^{k-1} \varphi\left(T^{* j} x\right) .
$$

Then $T^{* k} x=T^{\varphi_{k}(x)} x$ and

$$
\left[b_{\varphi_{k}(x)+1}, \ldots, b_{\varphi_{k}(x)+\nu}\right]=[\boldsymbol{\beta}] .
$$

For every $x \in X$ and $k \geq 1$,

$$
\left[b_{1}(x), \ldots, b_{\varphi_{k}(x)+\nu}(x)\right] \in \mathscr{R}(C, T),
$$

whence, by bounded distortion $(\nabla)$ and the Schweiger property,

$$
m\left(A \mid\left[b_{1}(x), \ldots, b_{\varphi_{k}(x)+\nu}(x)\right]\right)=C^{ \pm 3} m\left(A_{\varphi_{k}(x)+\nu} \mid T \beta_{\nu}\right) .
$$

It follows from the martingale convergence theorem that, for a.e. $x \in A$,

$$
m\left(A \mid\left[b_{1}(x), \ldots, b_{\varphi_{k}(x)+\nu}(x)\right]\right) \rightarrow 1_{A}(x) \text { as } k \rightarrow \infty,
$$

whence

$$
\liminf _{k \rightarrow \infty} m\left(A_{\varphi_{k}(x)+\nu} \mid T \beta_{\nu}\right)>0
$$

and

$$
\liminf _{k \rightarrow \infty} m\left(A_{\varphi_{k}(x)+\nu}\right)>0 .
$$

Since $\left.T^{*}\right|_{[\boldsymbol{\beta}]}$ is conservative and ergodic on $[\boldsymbol{\beta}], T^{*-1}[\boldsymbol{\beta}]=X$, and $m(A \cap[\boldsymbol{\beta}])>0$, it follows that

$$
\bigcup_{n=0}^{\infty} T^{*-n} A=X \quad \bmod m .
$$

Suppose $x \in X$ and $t \in \mathbb{N}$, are such that $T^{* t} x \in A$, and

$$
\liminf _{k \rightarrow \infty} m\left(A_{\varphi_{k}\left(T^{* 1} x\right)+\nu}\right)>0 \text {. }
$$

Then

$$
\exists \varepsilon>0 \ni m\left(A_{\varphi_{k}\left(T^{* t} x\right)+\nu}\right) \geq \varepsilon \quad \forall k \text { large. }
$$

Recall that

$$
A_{\varphi_{k+t}(x)+\nu}=A_{\varphi_{k}\left(T^{* t} x\right)+\varphi_{t}(x)+\nu}=T^{\varphi_{t}(x)} A_{\varphi_{k}\left(T^{* t} x\right)+\nu}
$$

Next, we claim that

$$
\exists \varepsilon_{1}>0 \ni B \in \mathscr{F}, m(B) \geq \varepsilon \Rightarrow m\left(T^{\varphi_{t}(x)} B\right) \geq \varepsilon_{1} .
$$


To see this note first that

$$
\exists \mathscr{R}_{\varepsilon} \subseteq \mathscr{R}_{0}^{\varphi_{t}(x)-1} \ni\left|\mathscr{R}_{\varepsilon}\right|<\infty \text { and } m\left(\bigcup_{b \in \mathscr{R}_{\varepsilon}} b\right) \geq 1-\varepsilon / 2 .
$$

The map $T^{\varphi_{t}(x)}$ is invertible and nonsingular on every $b \in \mathscr{R}_{\varepsilon}$, whence, since $\left|\mathscr{R}_{\varepsilon}\right|<\infty$,

$$
\forall \eta \eta^{\prime}>0 \quad \ni B \in \mathscr{F}, m(B \cap b) \geq \eta \text { for some } b \in \mathscr{R}_{\varepsilon} \Rightarrow m\left(T^{\varphi_{t}(x)} B\right) \geq \eta^{\prime} .
$$

It follows from the definition of $\mathscr{R}_{\varepsilon}$ that

$$
m(B) \geq \varepsilon \Rightarrow \exists b \in \mathscr{R}_{\varepsilon} \ni m(B \cap b) \geq \frac{\varepsilon}{2\left|\mathscr{R}_{\varepsilon}\right|}
$$

and the claim is established.

From the claim, we obtain that

$$
\liminf _{k \rightarrow \infty} m\left(A_{\varphi_{k}(x)+\nu}\right)>0 \text { a.e. on } X .
$$

To establish the theorem, it is sufficient (again, by the martingale convergence theorem) to prove that

$$
\limsup _{k \rightarrow \infty} m\left(A_{\varphi_{k}(x)+\nu} \cap T \beta_{\nu}\right)>0 \text { a.e. on } X .
$$

To see this, let $x \in X$ with $m\left(A_{\varphi_{k}(x)+\nu}\right) \geq \varepsilon>0$ for some $\varepsilon>0$ and all large $k$. By aperiodicity of $(T, \mathscr{R})$

$$
\exists p_{0} \in \mathbb{N} \ni m\left(T^{p} \beta_{\nu}\right) \geq 1-\varepsilon / 4, \quad \forall p \geq p_{0} .
$$

By ergodicity of $\left.T^{*}\right|_{[\beta]}$,

$$
\exists p_{1} \geq p_{0}, l \geq 1 \ni \sum_{k=1}^{\infty} 1_{\left[\varphi_{l}=p_{1}\right]} \circ T^{* k}=\infty \quad \text { a.e. on } X .
$$

As before,

$$
\exists \mathscr{R}_{p_{1}, \varepsilon} \subseteq \mathscr{R}_{0}^{p_{1}-1} \cap T \beta_{\nu} \ni\left|\mathscr{R}_{p_{1}, \varepsilon}\right|<\infty \text { and } m\left(\bigcup_{b \in \mathscr{R}_{p_{1}, \varepsilon}} T^{p_{1}} b\right) \geq 1-\varepsilon / 2,
$$

whence

$$
\exists b \in \mathscr{R}_{p_{1}, \varepsilon} \ni \quad m\left(A_{\varphi_{k}(x)+\nu} \cap T^{p_{1}} b\right) \geq \frac{\varepsilon}{2\left|\mathscr{R}_{p_{1}, \varepsilon}\right|}
$$

for large $k$. Also as before, there exists $\varepsilon_{1}>0$, such that

$$
\begin{aligned}
& \text { if } B \in \mathscr{F}, \text { and } b \in \mathscr{R}_{p_{1}, \varepsilon} \ni m\left(B \cap T^{p_{1}} b\right) \geq \frac{\varepsilon}{2\left|\mathscr{R}_{p_{1}, \varepsilon}\right|}, \text { then } \\
& m\left(T^{-p_{1}} B \cap b\right) \geq \varepsilon_{1}, \text { and hence } m\left(T^{-p_{1}} B \cap T \beta_{\nu}\right) \geq \varepsilon_{1} .
\end{aligned}
$$

This shows that

$$
m\left(A_{\varphi_{k}(x)-p_{1}+\nu} \cap T \beta_{\nu}\right)=m\left(T^{-p_{1}} A_{\varphi_{k}(x)+\nu} \cap T \beta_{\nu}\right) \geq \varepsilon_{1}
$$

for large $k$. 
Because of the choice of $p_{1}$, we have

$$
\begin{aligned}
\varepsilon_{1} & \leq m\left(A_{\varphi_{j+l}(x)-p_{1}+\nu} \cap T \beta_{\nu}\right) \\
& =m\left(A_{\varphi_{j}(x)+\varphi_{l}\left(T^{* j} x\right)-p_{1}+\nu} \cap T \beta_{\nu}\right)=m\left(A_{\varphi_{j}(x)+\nu} \cap T \beta_{\nu}\right)
\end{aligned}
$$

for infinitely many $j$, and

$$
\limsup _{k \rightarrow \infty} m\left(A_{\varphi_{k}(x)+\nu} \cap T \beta_{\nu}\right) \geq \varepsilon_{1} .
$$

This establishes the theorem. $\diamond$

\section{WANDERING RATES FOR MARKOV FIBRED SYSTEMS}

Suppose that $(T, \mathscr{R})$ is a conservative, ergodic fibred system having the Schweiger property with respect to $\mathscr{R}(C, T)$.

Let $A \in \mathscr{F}$. Set $A_{0}=A$ and

$$
A_{k}=T^{-k} A \backslash \bigcup_{j=0}^{k-1} T^{-j} A, \quad(k \geq 1), \quad c_{k}(A)=\mu\left(A_{k}\right), \quad(k \geq 0),
$$

where $\mu \sim m$ and $\mu \circ T^{-1}=\mu$. The wandering rate of $A$ is defined by

$$
L_{A}(n)=\mu\left(\bigcup_{l=0}^{n} T^{-l} A\right)=\sum_{k=0}^{n} c_{k}(A)
$$

(see [2] or [36]). The following is a generalisation of Theorem 3 of [36]. Let $\mathscr{F}$ denote the ring generated by $\mathscr{R}(C, T)$.

Theorem 4.1. Suppose that $(T, \mathscr{R})$ is a conservative, ergodic fibred system having the Schweiger property with respect to $\mathscr{R}(C, T)$. Then $\exists L(n) \uparrow$

$$
L_{B}(n) \sim L(n) \text { as } n \rightarrow \infty \forall B \in \mathscr{F}_{+}, B \subset A \in \mathscr{F}_{0} .
$$

Proof. It is sufficient to show that if $A \in \mathscr{F}$ is a finite union of sets in $\mathscr{R}(C, T)$, then

$$
L_{A}(n) \sim L_{B}(n) \text { as } n \rightarrow \infty, \forall B \in \mathscr{F} \cap A, m(B)>0 .
$$

The proof of this is based on Thaler's proof of Theorem 3 in [36], and uses

Lemma 4.2. Suppose that $(T, \mathscr{R})$ is a conservative, ergodic fibred system having the Schweiger property with respect to $\mathscr{R}(C, T)$, and suppose that $A \in \mathscr{F}$ is a finite union of sets in $\mathscr{R}(C, T)$. Then

$$
\mu\left(T^{-k} B \backslash \bigcup_{j=0}^{\exists M \geq 1} T^{-j} A\right) \leq M m(B) \mu\left(T^{-k} A \backslash \bigcup_{j=0}^{k-1} T^{-j} A\right) .
$$

Proof. First, since $A \in \mathscr{F}$ is a finite union of sets in $\mathscr{R}(C, T)$, by Theorem 3.1,

$$
\exists M \geq 1 \ni \frac{d \mu}{d m} \in[1 / M, M] \text { on } A \text {. }
$$

Also, since $T$ is conservative and ergodic, for $B \in \mathscr{F}$, we have by Kac's formula,

$$
\mu(B)=\sum_{l=0}^{\infty} \mu\left(A \cap T^{-l} B \backslash \bigcup_{j=1}^{l} T^{-j} A\right)
$$


Thus, for $B \in \mathscr{F} \cap A$ and $k \geq 1$,

$$
\begin{aligned}
\mu\left(T^{-k} B \backslash \bigcup_{j=0}^{k-1} T^{-j} A\right) & =\sum_{l=1}^{\infty} \mu\left(T^{-(k+l)} B \cap A \backslash \bigcup_{j=1}^{k+l-1} T^{-j} A\right) \\
& \leq M \sum_{l=1}^{\infty} m\left(T^{-(k+l)} B \cap A \backslash \bigcup_{j=1}^{k+l-1} T^{-j} A\right)
\end{aligned}
$$

Using bounded distortion $(\nabla)$ and the Schweiger property, it is not hard to see that

$$
\begin{aligned}
& \exists K \geq 1 \ni \forall B \in \mathscr{F} \cap A, \quad k, l \geq 1, \\
& m\left(T^{-(k+l)} B \cap A \backslash \bigcup_{j=1}^{k+l-1} T^{-j} A\right) \leq K m(B) m\left(T^{-(k+l)} A \cap A \backslash \bigcup_{j=1}^{k+l-1} T^{-j} A\right)
\end{aligned}
$$

whence

$$
\begin{aligned}
\mu\left(T^{-k} B \backslash \bigcup_{j=0}^{k-1} T^{-j} A\right) & \leq M K \sum_{l=1}^{\infty} m(B) m\left(T^{-(k+l)} A \cap A \backslash \bigcup_{j=1}^{k+l-1} T^{-j} A\right) \\
& \leq M^{2} K \sum_{l=1}^{\infty} m(B) \mu\left(T^{-(k+l)} A \cap A \backslash \bigcup_{j=1}^{k+l-1} T^{-j} A\right) \\
& =M^{2} K m(B) \mu\left(T^{-k} A \backslash \bigcup_{j=1}^{k-1} T^{-j} A\right) . \diamond
\end{aligned}
$$

Continuing the proof of Theorem 4.1 , let $B \in \mathscr{F} \cap A$. Then

$$
\bigcup_{k=0}^{n} T^{-k} A \backslash \bigcup_{k=0}^{n} T^{-k} B \subset(A \backslash B) \cup \bigcup_{k=1}^{n} T^{-k}(A \backslash B) \backslash \bigcup_{j=0}^{k-1} T^{-j} A,
$$

whence

$$
\begin{aligned}
L_{A}(n)-L_{B}(n) & \leq \mu(A \backslash B)+\sum_{k=1}^{n} \mu\left(T^{-k}(A \backslash B) \backslash \bigcup_{j=0}^{k-1} T^{-j} A\right) \\
& \leq M m(A \backslash B) L_{A}(n)
\end{aligned}
$$

by Lemma 4.2. Using this, we obtain for any fixed $d \geq 1$,

$$
\begin{aligned}
L_{B}(n) & \sim L_{B}(n+d) \quad \text { as } n \rightarrow \infty \\
& \geq L_{A \cap \bigcup_{j=0}^{d} T^{-j} B}(n) \\
& \geq\left(1-M m\left(A \backslash \bigcup_{j=0}^{d} T^{-j} B\right)\right) L_{A}(n) .
\end{aligned}
$$

This establishes Theorem 4.1 since

$$
m\left(A \backslash \bigcup_{j=0}^{d} T^{-j} B\right) \rightarrow 0 \text { as } d \rightarrow \infty . \diamond
$$


Recall that if $(T, \mathscr{R})$ is a fibred system, then, for $p \geq 1$, so is $\left(T^{p}, \mathscr{R}_{0}^{p-1}\right)$. If $(T, \mathscr{R})$ is aperiodic, then so is $\left(T^{p}, \mathscr{R}_{0}^{p-1}\right)$. If $(T, \mathscr{R})$ has the Schweiger property, then so does $\left(T^{p}, \mathscr{R}_{0}^{p-1}\right)$. Clearly, it can be fixed that

$$
\mathscr{R}\left(C, T^{p}\right) \subseteq \mathscr{F}_{0}(T) .
$$

From this follows

Proposition 4.3. Suppose that $(T, \mathscr{R})$ is an aperiodic, conservative fibred system having the Schweiger property with respect to $\mathscr{R}(C, T)$. Then

$$
L\left(T^{p}, n\right) \sim L(T, p n) \text { as } n \rightarrow \infty \forall p \geq 1 .
$$

Next we recall the relationship between wandering rates and return sequences for a conservative ergodic fibred system $(T, \mathscr{R})$ with the Schweiger property. Suppose that the return sequence for $T$ is given by

$$
a_{n}(T) \sim \sum_{k=1}^{n} u_{k} \quad \text { as } n \rightarrow \infty
$$

where $u_{k} \geq 0 \forall k \in \mathbb{N}$. It follows from Theorem 3.1 and the asymptotic renewal equation (see [3]) that

$$
\sum_{n=0}^{\infty} u_{n} x^{n} \sim \frac{1}{1-x} \frac{1}{c_{A}(x)} \quad \text { as } x \uparrow 1^{-}, \quad \forall A \in \mathscr{F}, A \subseteq B \in \mathscr{R}(C, T),
$$

where

$$
c_{A}(x)=\sum_{k=0}^{\infty} c_{k}(A) x^{k}
$$

In case $L(n):=\sum_{k=0}^{n} c_{k}$ is regularly varying with index $\alpha \in[0,1)$, it follows from Karamata's Tauberian theorem that

$$
a_{n}(T) \sim \frac{1}{\Gamma(1+\alpha) \Gamma(2-\alpha)} \frac{n}{L(n)} \quad \text { as } n \rightarrow \infty .
$$

In $\S 9$, this will be used to identify the return sequences of parabolic rational maps from their wandering rates, which will be calculated (also in §9) using results established in the next section.

\section{PARABOlic FIBRED SYSTEMS}

Suppose that $(T, \mathscr{R})$ is a fibred system having the Schweiger property with respect to $\mathscr{R}(C, T)$. It follows from the definition of $\mathscr{R}(C, T)$ that $N_{C} \circ T \geq$ $N_{C}-1$ whenever $N_{C} \geq 2$.

In this section, we assume that the fibred system is irreducible, and that $|T \mathscr{R}|<\infty$. We also assume that the collection $\mathscr{R}(C, T)$ satisfies the additional properties that

$$
\begin{gathered}
N_{C} \circ T=N_{C}-1 \quad\left(N_{C} \geq 2\right), \\
\left|\mathscr{R}_{0}^{1} \cap\left[N_{C}=2\right]\right|<\infty, \\
T:\left[N_{C} \geq 2\right] \rightarrow T\left[N_{C} \geq 2\right] \quad \text { is invertible },
\end{gathered}
$$

and also

$$
T\left(\left[N_{C}=1\right] \backslash T\left[N_{C}=2\right]\right)=X .
$$


These assumptions are satisfied by parabolic rational maps, as will be seen in the sequel, and, accordingly, we shall call a fibred system satisfying them parabolic (with respect to $\mathscr{R}(C, T)$ ).

If $(T, \mathscr{R})$ is a parabolic fibred system, then, by Theorems 2.8 and 3.1, $T$ is conservative and ergodic, and there is a $\sigma$-finite, $T$-invariant measure $\mu$ on $X$ equivalent to $m$. Let $P=\left[N_{C} \geq 2\right]$. Then $T: P \rightarrow T(P)$ is nonsingular and invertible, and we obtain that there exists a unique inverse branch of $T$,

$$
f_{*}: T(P) \rightarrow P .
$$

Clearly, $f_{*}(P) \subset P$ (since $\left.T P \supset P\right)$. Whence, for $n \geq 1$,

$$
f_{*}^{n}: T(P) \rightarrow P
$$

is defined.

Since $|(T P \backslash P) \cap \mathscr{R}|<\infty$, it follows that for every $a \in(T P \backslash P) \cap \mathscr{R}$ there is an eventually periodic sequence $\left\{b_{n}\right\}_{n=1}^{\infty}\left(b_{n}=b_{n}(a)\right)$ such that

$$
f_{*}^{n} a=\left[b_{n}, \ldots, b_{1}, a\right] \quad(n \geq 1) .
$$

Writing

$$
\left(b_{1}, \ldots, b_{n}, \ldots\right)=\left(b_{1}, \ldots, b_{r}, \overline{\beta_{1}, \ldots, \beta_{d}}\right),
$$

where $r=r(a)$ and $d=d(a)$, we have $b_{k d+r+\nu}=\beta_{\nu}(k \geq 0,0 \leq \nu \leq d-1)$, whence

$$
f_{*}^{d k+r+\nu} a \subseteq\left[\beta_{\nu}, \ldots, \beta_{1}, \beta_{d}, \ldots, \beta_{\nu+1}\right] \quad \forall k \geq 0,0 \leq \nu \leq d-1 .
$$

Lemma 5.1. Suppose that $(T, \mathscr{R})$ is a parabolic fibred system with respect to $\mathscr{R}(C, T)$. Then $X^{*}=X$ and $\left(T^{*}, \mathscr{R}^{*}\right)$ is irreducible.

Proof. Note first that if $x, y \in X, N_{C}(x)=1$, and $T^{n} y=x$, then

$$
\exists k \leq n \ni T^{* k} y=x \text {. }
$$

It follows from irreducibility and the Markov property of $(T, \mathscr{R})$ that

$$
\forall B \in \bigcup_{k=1}^{\infty} \mathscr{R}_{0}^{n-1}, b \in \mathscr{R} \cap\left[N_{C}=1\right], \quad \exists n \geq 1 \ni T^{* n} B \supseteq b .
$$

It now follows from the equality $T\left(\left[N_{C}=1\right] \backslash T\left[N_{C}=2\right]\right)=X$ that

$$
\forall B, B^{\prime} \in \bigcup_{k=1}^{\infty} \mathscr{R}_{0}^{n-1}, \quad \exists n \geq 1 \ni T^{* n} B \supseteq B^{\prime},
$$

which yields $X^{*}=X$ and the irreducibility of $\left(T^{*}, \mathscr{R}^{*}\right) . \diamond$

By Lemma 2.1, there is a $T^{*}$-invariant probability $q \sim m$ such that $\log \frac{d q}{d m} \in$ $L^{\infty}(m)$. It is not hard to show that if

$$
\mu^{\prime}(B):=\sum_{k=0}^{\infty} q\left(T^{-k} B \cap\left[N_{C}>k\right]\right),
$$

then $\mu^{\prime} \circ T^{-1}=\mu^{\prime} \ll m$, whence, by conservativity and ergodicity of $T$, $\mu=c \mu^{\prime}$, where $c \in \mathbb{R}_{+}$is a constant. We assume that $c=1$, that is,

$$
\mu(B):=\sum_{k=0}^{\infty} q\left(T^{-k} B \cap\left[N_{C}>k\right]\right)
$$


It follows from (\$) that

$$
\mu\left(\left[N_{C} \leq \nu\right]\right) \leq \nu \quad \forall \nu \in \mathbb{N} .
$$

Lemma 5.2. Suppose that $(T, \mathscr{R})$ is a parabolic fibred system with respect to $\mathscr{R}(C, T)$. Then

$$
L_{A}(n)=\mu\left(\left[N_{C} \leq n\right]\right)+O(1) \text { as } n \rightarrow \infty,
$$

where $A=\left[N_{C}=\nu\right](\nu \geq 2)$ and $\mu \sim m, \mu \circ T^{-1}=\mu$.

Proof. Set $A_{0}=A$ and $A_{k}=T^{-k} A \backslash \bigcup_{j=0}^{k-1} T^{-j} a(k \geq 1)$. Then $L_{A}(n)=$ $\sum_{k=0}^{n} \mu\left(A_{k}\right)$ and $A_{k}=\left[N_{C}=\nu+k\right] \cup B_{k}$, where $B_{k} \subset\left[N_{C}<\nu\right]$ are disjoint. Thus,

$$
\begin{aligned}
L_{A}(n) & =\sum_{k=0}^{n} \mu\left(\left[N_{C}=\nu+k\right]\right)+\mu\left(\bigcup_{k=0}^{n} B_{k}\right) \\
& =\mu\left(\left[N_{C} \leq n\right]\right)+O(1) . \diamond
\end{aligned}
$$

Lemma 5.3. Suppose that $(T, \mathscr{R})$ is a parabolic fibred system with respect to $\mathscr{R}(C, T)$. Then

$$
\mu\left(\left[N_{C} \leq n\right]\right)=\int_{X}\left(N_{C} \wedge n\right) d q+O(1) \text { as } n \rightarrow \infty .
$$

Proof. For $n \geq 2$ and $k \geq 1$,

$$
\left[2+k \leq N_{C} \leq n+k\right] \subseteq T^{-k}\left[N_{C} \leq n\right] \subseteq\left[N_{C} \leq n+k\right]
$$

hence

$q\left(\left[k+2 \leq N_{C} \leq k+n\right]\right) \leq q\left(T^{-k}\left[N_{C} \leq n\right] \cap\left[N_{C}>k\right]\right) \leq q\left(\left[k+1 \leq N_{C} \leq k+n\right]\right)$ and

$$
\begin{aligned}
\mu\left(\left[N_{C} \leq n\right]\right) & =\sum_{k=0}^{\infty} q\left(T^{-k}\left[N_{C} \leq n\right] \cap\left[N_{C}>k\right]\right) \\
& =\sum_{k=0}^{\infty} q\left(\left[k+1 \leq N_{C} \leq k+n\right]\right)+O(1) \\
& =\sum_{k=1}^{\infty} k \wedge n q\left(\left[N_{C}=k\right]\right)+O(1) \\
& =\int_{X}\left(N_{C} \wedge n\right) d q+O(1), \quad \text { as } n \rightarrow \infty . \diamond
\end{aligned}
$$

For $x \in T(P)$ we denote

$$
\partial_{n}(x)=\frac{d m \circ f_{*}^{n}}{d m}
$$

and

$$
\Delta_{n}(x)=\Delta\left(b_{1}, \ldots, b_{n}\right)\left(f_{*}^{n}(x)\right),
$$

where $f_{*}^{n}(x) \in\left[b_{1}, \ldots, b_{n}\right] \in \mathscr{R}_{0}^{n}$. Clearly $\Delta_{n}(x) \partial_{n}(x)=1$. 
Lemma 5.4. Suppose that $(T, \mathscr{R})$ is a parabolic fibred system with respect to $\mathscr{R}(C, T)$. Then

$$
\begin{gathered}
\forall \nu \geq 2, B \in\left[N_{C}=\nu\right] \cap \mathscr{R}_{0}^{\nu-1}, \quad \forall n \geq 1, \text { for a.e. } x \in B \\
\sum_{k=0}^{\infty}(k \wedge n) m\left(f_{*}^{k} B\right)=\operatorname{Dm}(B) \sum_{k=0}^{\infty}(k \wedge n) \partial_{k}(x),
\end{gathered}
$$

where $D \in\left[C^{-2}, C^{2}\right]$.

Proof. First, note that if [b] $\in \mathscr{R}_{0}^{\nu-1} \cap\left[N_{C}=\nu\right]$, then [b] $\in \mathscr{R}(C, T)$, and there exist $b_{1}, b_{2}, \ldots \in \mathscr{R}$ such that

$$
f_{*}^{n}([\mathbf{b}])=\left[b_{n}, \ldots, b_{1}, \mathbf{b}\right] \quad(n \geq 1) .
$$

Clearly, $f_{*}^{n}([\mathbf{b}]) \in \mathscr{R}(C, T)$ and hence

$$
\begin{gathered}
\text { for a.e. } x \in[\mathbf{b}], n \geq 0 \exists D \in\left[1 / C^{2}, C^{2}\right] \ni \\
\partial_{n}(x)=D \frac{m\left(f_{*}^{n}[\mathbf{b}]\right)}{m([\mathbf{b}])} .
\end{gathered}
$$

Thus for a.e. $x \in[\mathbf{b}] \in \mathscr{R}_{0}^{\nu-1} \cap\left[N_{C}=\nu\right]$ and $n \geq 1$,

$$
\begin{aligned}
\sum_{k=0}^{\infty}(k \wedge n) m\left(f_{*}^{k}[\mathbf{b}]\right) & =\sum_{k=0}^{\infty}(k \wedge n) \int_{[\mathbf{b}]} \partial_{k} d m \\
& =D m([\mathbf{b}]) \sum_{k=0}^{\infty}(k \wedge n) \partial_{k}(x),
\end{aligned}
$$

where $D \in\left[C^{-2}, C^{2}\right] . \diamond$

Theorem 5.5. Suppose that $(T, \mathscr{R})$ is a parabolic fibred system with respect to $\mathscr{R}(C, T)$, and let $\nu \geq 2$ and $K=\mathscr{R}_{0}^{\nu-1} \cap\left(\left[N_{C}=\nu\right]\right)$. Then

$$
\begin{gathered}
\exists M>1 \ni \quad \text { for a.e. }\left(x_{b}\right)_{b \in K} \in \prod_{b \in K} b, \quad \forall n \in \mathbb{N} \\
M^{-1} L(n) \leq \sum_{b \in K} \sum_{k=0}^{\infty}(k \wedge n) \partial_{k}\left(x_{b}\right) \leq M L(n) .
\end{gathered}
$$

In particular,

$$
\mu(X)<\infty \Leftrightarrow \sum_{n=0}^{\infty} n \partial_{n}(x)<\infty \text { for a.e. } x \in T(P) .
$$

Proof. By Lemmas 5.1, 5.2, and 5.3,

$$
\begin{aligned}
L(n) & \sim \sum_{k=0}^{\infty}(k \wedge n) q\left(\left[N_{C}=k\right]\right) \sim \sum_{k=0}^{\infty}(k \wedge n) q\left(f_{*}^{k}\left[N_{C}=\nu\right]\right) \\
& =D \sum_{k=0}^{\infty}(k \wedge n) m\left(f_{*}^{k}\left[N_{C}=\nu\right]\right)=D \sum_{B \in K} \sum_{k=0}^{\infty}(k \wedge n) m\left(f_{*}^{k} B\right) \\
& =D E \sum_{B \in K} \sum_{k=0}^{\infty}(k \wedge n) \partial_{k}\left(x_{B}\right),
\end{aligned}
$$


where $|\log D| \leq\left\|\log \frac{d q}{d m}\right\|_{L^{\infty}(m)}$ and $E \in\left[C^{-2}, C^{2}\right]$. This proves the first part of the theorem. The second part follows since, clearly, $\mu(X)=\lim _{n \rightarrow \infty} L(n) . \diamond$

Proposition 5.6. Suppose that $(T, \mathscr{R})$ is a parabolic fibred system such that $\mu(X)=\infty$, and suppose that $\frac{d q}{d m}$ is uniformly continuous on $\mathscr{R}^{\mathbb{N}}$ in the sense that

$$
\begin{aligned}
& \forall \varepsilon>0, \exists n \geq 1 \ni \\
& \left|\frac{d q}{d m}(x)-\frac{d q}{d m}(y)\right|<\varepsilon \quad \forall x, y \in X \quad \ni b_{k}(x)=b_{k}(y) \quad(1 \leq k \leq n) .
\end{aligned}
$$

Then

$$
L(n) \sim \sum_{a \in T P \backslash P \cap \mathscr{R}} \sum_{\nu=0}^{d(a)-1} c_{a, \nu} \sum_{k=1}^{\infty}(k \wedge n) m\left(f_{*}^{d k+r+\nu} a\right) \text { as } n \rightarrow \infty,
$$

where $c_{a, \nu}>0 \forall a, \nu$, and where $r=r(a)$ and $d(a)$ are as defined before Lemma 5.1.

Proof. By Lemmas 5.2 and 5.3,

$$
\begin{aligned}
L(n) & \sim \sum_{k=1}^{\infty} k \wedge n q\left(\left[N_{C}=k\right]\right) \sim \sum_{k=1}^{\infty} k \wedge n q\left(f_{*}^{k}(T P \backslash P)\right) \\
& \sim \sum_{a \in T P \backslash P \cap \mathscr{R}} \sum_{k=1}^{\infty}(k \wedge n) q\left(f_{*}^{k} a\right) \\
& \sim \sum_{a \in T P \backslash P \cap \mathscr{R}} d(a) \sum_{\nu=0}^{d(a)-1} \sum_{k=1}^{\infty}(k \wedge n) q\left(f_{*}^{d k+r+\nu} a\right) \quad \text { as } n \rightarrow \infty .
\end{aligned}
$$

Since

$$
f_{*}^{d k+r+\nu}(x) \rightarrow\left(\overline{\beta_{\nu}, \ldots, \beta_{1}, \beta_{d}, \ldots, \beta_{\nu+1}}\right) \text { in } \mathscr{R}^{\mathbb{N}}
$$

for $x \in a$ and $0 \leq \nu \leq d-1$, it follows by the continuity of $\frac{d q}{d m}$ that

$$
\begin{gathered}
\forall a \in T P \backslash P \cap \mathscr{R}, 0 \leq \nu \leq d-1, \exists c_{a, \nu}>0 \ni \\
\frac{d q}{d m} \circ f_{*}^{d k+r+\nu}(x) \rightarrow c_{a, \nu} \text { as } k \rightarrow \infty \forall x \in a,
\end{gathered}
$$

and hence

$$
q\left(f_{*}^{d k+r+\nu} a\right) \sim c_{a, \nu} m\left(f_{*}^{d k+r+\nu} a\right) \quad \text { as } k \rightarrow \infty .
$$

The proposition follows from this. $\diamond$

\section{MIXING PROPERTIES OF FIBERED SYSTEMS WITH FINITE INVARIANT MEASURES}

The first goal of this section is to establish

Theorem 6.1. Suppose that $(T, \mathscr{R})$ is an aperiodic fibred system such that for some $C \in \mathbb{R}_{+}$,

$$
\mathscr{G}(C, T)=\widetilde{\mathscr{R}}_{+},
$$

there exist $\mathscr{R}_{0}, \mathscr{R}_{1} \subseteq \mathscr{R}$ such that $\left|\mathscr{R}_{0}\right|<\infty$, and $T X_{1} \subseteq X_{0}$, where $X_{i}=$ $\bigcup_{B \in \mathscr{R}_{i}} B(i=0,1)$. Then there is a $T$-invariant probability $q \sim m$ such that $\log \frac{d q}{d m} \in L^{\infty}(m)$, and $(T, \mathscr{R})$ is continued fraction mixing.

The proof is presented in a sequence of lemmas. 
Note that under the assumptions of Theorem 6.1, since $T X=X \bmod m$, necessarily $T X_{0} \subseteq X_{1} \bmod m$. Also, $|\{T b: b \in \mathscr{R}\}|<\infty$, and so by Lemma 2.1 there is a $T$-invariant probability $q \sim m$ such that $\log \frac{d q}{d m} \in L^{\infty}(m)$.

Lemma 6.2. Under the assumptions of Theorem 6.1,

$$
\begin{gathered}
\exists L \in \mathbb{R}_{+} \ni \forall n \in \mathbb{N}, \quad A \in \mathscr{R}_{0}^{n-1}, B \in \widetilde{\mathscr{R}}: \\
\text { either } q\left(A \cap T^{-n} B\right)=0, \text { or } \frac{1}{L} \leq \frac{q\left(A \cap T^{-n} B\right)}{q(A) q(B)} \leq L .
\end{gathered}
$$

Proof. If $A=\left[\right.$ a] $\in \mathscr{R}_{0}^{n-1}, B \in \widetilde{\mathscr{R}}$, and $m\left(A \cap T^{-n} B\right)>0$, then, by bounded distortion $(\nabla)$,

$$
C^{-3} m\left(T a_{n}\right)^{-1} \leq \frac{m\left(A \cap T^{-n} B\right)}{m(A) m(B)} \leq C^{3} m\left(T a_{n}\right)^{-1} .
$$

The result follows from this since $|\{T b: b \in \mathscr{R}\}|<\infty$ and $\log \frac{d q}{d m} \in L^{\infty}(m) . \diamond$ Remark. In case $|\mathscr{R}|<\infty$, the continued fraction mixing of $(T, \mathscr{R})$ follows from Lemma 6.2 and Corollary 2.4 of [12].

Lemma 6.3. Under the assumptions of Theorem 6.1,

$$
\exists n_{0} \in \mathbb{N} \ni T^{n} B=X \bmod m \quad \forall B \in \mathscr{R}, n \geq n_{0} .
$$

Proof. Since $\left|\mathscr{R}_{0}\right|<\infty$, by aperiodicity

$$
\exists N \in \mathbb{N} \ni m\left(B \cap T^{-n} B^{\prime}\right)>0 \quad \forall n \geq N, B, B^{\prime} \in \mathscr{R}_{0},
$$

whence, by the Markov property,

$$
X_{0} \subseteq T^{n} B \bmod m \quad \forall n \geq N, B \in \mathscr{R}_{0} .
$$

Since $T B \subseteq X_{0} \forall B \in \mathscr{R}$,

$$
X_{0} \subseteq T^{n} B \quad \forall n \geq N+1, B \in \mathscr{R}
$$

and, since $T X_{0} \supset X_{1}$,

$$
X \subseteq T^{n} B \bmod m \quad \forall n \geq N+2, B \in \mathscr{R} . \diamond
$$

For $b \in \mathscr{R}_{0}$ and $b^{\prime} \in \mathscr{R}_{1}$ such that $b^{\prime} \subset T b$ set

$$
\mathscr{C}\left(b, b^{\prime}\right)=\left\{c \in \mathscr{R}_{1}: T(c)=T\left(b^{\prime}\right), c \subset T(b)\right\} .
$$

Remarks. Suppose $\varnothing \neq\left[b_{1}, \ldots, b_{n}\right] \in \mathscr{R}_{0}^{n-1}$. Then

$$
b_{i} \in \mathscr{R}_{1} \Rightarrow b_{i-1}, b_{i+1} \in \mathscr{R}_{0} \text {, }
$$

since $T X_{1} \subseteq X_{0}$ and

$b_{j} \in \mathscr{R}_{0}, b_{j+1} \in \mathscr{R}_{1}, b \in \mathscr{C}\left(b_{j}, b_{j+1}\right) \Rightarrow\left[b_{1}, \ldots, b_{j}, b, b_{j+2}, \ldots, b_{n}\right] \neq \varnothing$.

Lemma 6.4. Under the assumptions of Theorem 6.1,

$$
\exists \eta>0 \ni \forall A \in \mathscr{R}_{0}, B \in \mathscr{R}_{1}, B \subset T A \text { : }
$$

$$
m\left(\bigcup_{C \in \mathscr{C}(A, B)} C\right) \geq \eta \text {. }
$$


Proof. $\left|\left\{\mathscr{C}(A, B): A \in \mathscr{R}_{0}, B \in \mathscr{R}_{1}, B \subset T A\right\}\right|<\infty$.

If $A \in \mathscr{R}_{0}^{n-1}, B \in \widetilde{\mathscr{R}}$, and $p \in \mathbb{N}$, define

$$
\mathscr{D}_{p}(A, B)=\left\{C \in \mathscr{R}_{0}^{p-1}: A \cap T^{-n} C \cap T^{-n-p} B \neq \varnothing\right\}
$$

and

$$
D_{p}(A, B)=\bigcup_{C \in \mathscr{D}_{p}(A, B)} C .
$$

Lemma 6.5. Under the assumptions of Theorem 6.1,

$$
\begin{aligned}
& \forall p \in \mathbb{N} \ni T^{p} B=X \bmod m \forall B \in \mathscr{R}, \\
& \exists \gamma_{p}>0 \quad \ni m\left(D_{p}(A, B)\right) \geq \gamma_{p} \quad \forall A, B \in \widetilde{\mathscr{R}} .
\end{aligned}
$$

Proof. Suppose $A=\left[b_{1}, \ldots, b_{n}\right]$ and $B \in \widetilde{R}$, and recall that $T^{p+n} A=$ $X, \quad A \cap T^{-p} B \neq \varnothing$, and $\mathscr{D}_{p}(A, B) \neq \varnothing$. If

$$
[\mathbf{c}]=\left[c_{1}, \ldots, c_{p}\right] \in \mathscr{D}_{p}(A, B),
$$

then, by bounded distortion $(P)$,

$$
m\left(D_{p}(A, B)\right) \geq m([\mathbf{c}]) \geq \frac{1}{D^{p+1}} \prod_{k=1}^{p} m\left(c_{k}\right),
$$

where $D=C^{3} / \min \{m(T b): b \in \mathscr{R}\}$, and if

$$
\exists[\mathbf{c}]=\left[c_{1}, \ldots, c_{p}\right] \in \mathscr{D}_{p}(A, B) \ni c_{k} \in \mathscr{R}_{0} \quad \forall 1 \leq k \leq p,
$$

then

where $\varepsilon=\min _{B \in \mathscr{R}_{0}} m(B)$.

$$
m\left(D_{p}(A, B)\right) \geq \frac{\varepsilon^{p}}{D^{p+1}},
$$

If not, then $\exists[c]=\left[c_{1}, \ldots, c_{p}\right] \in \mathscr{D}_{p}(A, B)$ so that $c_{k} \in \mathscr{R}_{1}$ for some $k$. If $c_{j} \in \mathscr{R}_{0}, c_{j+1} \in \mathscr{R}_{1}$, and $r \in \mathscr{C}\left(c_{j}, c_{j+1}\right)$, then

$$
\left[c_{1}, \ldots, c_{j}, r, c_{j+2}, \ldots, c_{p}\right] \in \mathscr{D}_{p}(A, B) .
$$

If $c_{1} \in \mathscr{R}_{1}$, then $a_{n} \in \mathscr{R}_{0}$, and, for $r \in \mathscr{C}\left(a_{n}, c_{1}\right),\left[a_{n}, r, c_{2}, \ldots, c_{p}\right] \in$ $\mathscr{D}_{p}(A, B)$.

Set $L=\left\{1 \leq j \leq p: c_{j} \in \mathscr{R}_{1}\right\}$. Then, denoting $c_{0}=a_{n}$,

$$
\mathscr{D}_{p}(A, B) \supseteq\left\{\left[c_{1}^{\prime}, \ldots, c_{p}^{\prime}\right]: c_{i}^{\prime}=c_{i} \forall i \notin L, c_{i}^{\prime} \in \mathscr{C}\left(c_{i-1}, c_{i}\right) \forall i \in L\right\}=: \mathscr{D}(\mathbf{c}) .
$$

Hence

$$
m\left(D_{p}(A, B)\right) \geq \sum_{\left[\mathbf{c}^{\prime}\right] \in \mathscr{D}(\mathbf{c})} m\left(\left[\mathbf{c}^{\prime}\right]\right)=\frac{1}{D^{p+1}} \eta^{|L|} \varepsilon^{p-|L|} \geq \gamma_{p},
$$

where $\eta$ is as in Lemma 6.4 , and

$$
\gamma_{p}=\frac{1}{D}\left(\frac{\min \{\eta, \varepsilon\}}{D}\right)^{p} \cdot \diamond
$$

Lemma 6.6. Under the assumptions of Theorem 6.1,

$$
\exists L \in \mathbb{R}_{+}, p \in \mathbb{N} \ni \forall n \in \mathbb{N}, A \in \mathscr{R}_{0}^{n-1}, B \in \widetilde{\mathscr{R}}:
$$




$$
\frac{1}{L} \leq \frac{q\left(A \cap T^{-(n+p)} B\right)}{q(A) q(B)} \leq L .
$$

Proof. The upper bound follows from Lemma 6.2.

For $p \in \mathbb{N}$ such that $T^{p} B=X \forall B \in \mathbb{R}$,

$$
\begin{aligned}
m\left(A \cap T^{-(p+n)} B\right) & \geq D^{-3} m(A) m\left(D_{p}(A, B)\right) m(B) \\
& \geq \frac{\gamma_{p}}{D^{3}} m(A) m(B) \quad \forall n \in \mathbb{N}, A \in \varepsilon_{0}^{n-1}, B \in \widetilde{\mathscr{R}} .
\end{aligned}
$$

The result follows since $\log \frac{d q}{d m} \in L^{\infty}(m) . \diamond$

Proof of Theorem 6.1. The continued fraction mixing of $(T, \mathscr{R})$ follows from Lemma 6.6 by [9], and Theorem 6.1 is established.

\section{Central limit theorem}

In this section we assume that $(T, \mathscr{R})$ is a finite aperiodic and parabolic fibred system with respect to $\mathscr{R}(C, T)$, and that the $T$-invariant measure $\mu$ is finite. In particular,

$$
\mu(A)=\sum_{u=0}^{\infty} q\left(\left[N_{C}>u\right] \cap T^{-u} A\right) \quad(A \in \mathscr{F}) .
$$

Let $\mu_{0}=\mu / \mu(X)$ denote the equivalent probability measure. Under the assumptions, the jump transformation need not to be aperiodic as well. However, it follows that the other hypothesis in Theorem 6.1 is satisfied by setting $\mathscr{R}_{0}^{*}=\mathscr{R} \cap\left[N_{C}=1\right]$.

Theorem 7.1. Let $(T, \mathscr{R})$ be a finite aperiodic and parabolic fibred system with respect to $\mathscr{R}(C, T)$, and let there be two periodic points $x, y \in X$ with relatively prime periods $p$ and $p^{\prime}$ respectively, such that their forward orbits are contained in $\left[N_{C}=1\right]$. Then $\left(T^{*}, \mathscr{R}^{*}\right)$ is aperiodic and continued fraction mixing.

Proof. By Lemma 5.1, $\left(T^{*}, \mathscr{R}^{*}\right)$ is irreducible. Let $T^{j} x \in A_{j} \in \mathscr{R}(0 \leq j<p)$ and $T^{i} y \in B_{i} \in \mathscr{R}\left(0 \leq i<p^{\prime}\right)$. Then, by the Markov property,

$$
\begin{array}{lll}
A_{j+1} \subseteq T A_{j}, & A_{0} \subseteq T A_{p-1} & (0 \leq j \leq p-2), \\
B_{i+1} \subseteq T B_{i}, & B_{0} \subseteq T B_{p^{\prime}-1} & \left(0 \leq i \leq p^{\prime}-2\right) .
\end{array}
$$

By irreducibility there exist $n, m \geq 1$ so that $A_{0} \subseteq\left(T^{*}\right)^{n} B_{0}$ and $B_{0} \subseteq$ $\left(T^{*}\right)^{m} A_{0}$. Since $T=T^{*}$ on $\left[N_{C}=1\right]$ it follows that

$$
A_{0} \subseteq\left(T^{*}\right)^{p} A_{0} \cap\left(T^{*}\right)^{n+m} A_{0} \cap\left(T^{*}\right)^{m+n+p^{\prime}} A_{0}
$$

Since the common divisor of $p, n+m, n+m+p^{\prime}$ is $1,\left(T^{*}, \mathscr{R}^{*}\right)$ is aperiodic. By Theorem 6.1 it then follows that $\left(T^{*}, \mathscr{R}^{*}\right)$ is continued fraction mixing. $\diamond$

By Lemma 5.3, $A:=\int N_{C} d q=\mu(X)<\infty$, and by the ergodic theorem

$$
\frac{N_{n}}{n} \rightarrow \frac{1}{A} \text { a.e. }
$$

where

$$
N_{n}(x)=\min \left\{l: \sum_{i=0}^{l} N_{C}\left(\left(T^{*}\right)^{i} x\right) \geq n\right\}
$$


Let $f \in L^{\infty}(\mu)$ and define

$$
f^{*}(x)=f(x)+f(T x)+\cdots+f\left(T^{N_{C}(x)-1} x\right)-N_{C}(x) \int_{X} f d \mu_{0} \quad(x \in X) .
$$

It follows immediately that

$$
\mu(f):=\int_{X} f d \mu=\int_{X} \sum_{k=0}^{N_{C}-1} f \circ T^{k} d q,
$$

hence $q\left(f^{*}\right)=\int_{X} f^{*} d q=0$, and that $f^{*} \in L^{2}(q)$ in case

$$
\sum_{n \geq 1} n^{2} q\left(\left[N_{C}=n\right]\right)<\infty \text {. }
$$

Denote

$$
\sigma_{n}^{2}\left(f^{*}\right)=\sigma_{n}^{2}(f)=\int_{X}\left(\sum_{k=0}^{n} f^{*} \circ\left(T^{*}\right)^{k}\right)^{2} d q .
$$

In this section we prove

Theorem 7.2. Let $\left(T^{*}, \mathscr{R}^{*}\right)$ be absolutely regular with mixing coefficients $\beta(n):=\sup \left\{\int \sup \left\{|q(E \mid F)-q(E)|: F \in\left(\mathscr{F}^{*}\right)_{k+n}^{\infty}\right\} d q: E \in\left(\mathscr{F}^{*}\right)_{0}^{k} ; k \geq 0\right\}$ satisfying

$$
\sum_{n \geq 1} \beta(n)^{\zeta /(2+\zeta)}<\infty
$$

for some $\zeta>0$. Let $f$ be a bounded measurable function such that $f^{*} \in L^{\infty}(q)$ and such that

$$
\xi_{n}:=\left\|f^{*}-E_{q}\left(f^{*} \mid\left(\mathscr{F}^{*}\right)_{0}^{n}\right)\right\|_{L^{2+\zeta(q)}} \leq C n^{-2-\zeta} \quad(n \geq 1)
$$

for some constant $C>0$. Then

$$
c_{f}=q\left(\left(f^{*}\right)^{2}\right)+2 \sum_{l=1}^{\infty} q\left(f^{*} f^{*} \circ\left(T^{*}\right)^{l}\right)<\infty
$$

converges absolutely, $\lim _{n \rightarrow \infty} \sigma_{n}^{2} / n=\lim _{n \rightarrow \infty} \sigma_{n}^{2}\left(f^{*}\right) / n=c_{f}$, and, if $c_{f} \neq 0$, then $f$ satisfies the central limit theorem, that is,

$$
\lim _{n \rightarrow \infty} \mu_{0}\left(\left[\frac{\sqrt{A}}{\sqrt{n c_{f}}} \sum_{k=0}^{n}\left(f \circ T^{k}-\mu_{0}(f)\right) \leq t\right]\right)=\frac{1}{\sqrt{2 \pi}} \int_{-\infty}^{t} \exp \left(-u^{2} / 2\right) d u
$$

for any $t \in \mathbb{R}$.

Corollary 7.3. Let $\left(T^{*}, \mathscr{R}^{*}\right)$ be aperiodic, and let $f$ be measurable with respect to $\sigma\left(\mathscr{R}_{0}^{m}\right)$ for some $m \geq 0$ such that $f^{*} \in L^{2}(q)$. If $\sigma_{n}^{2}=\sigma_{n}^{2}\left(f^{*}\right) \rightarrow \infty$, then $\sigma_{n}^{2}=n h(n)$, where $h$ is slowly varying on $\mathbb{R}$ at infinity, and $f$ satisfies the central limit theorem, that is,

$$
\lim _{n \rightarrow \infty} \mu_{0}\left(\left[\frac{\sqrt{A}}{\sigma_{n}} \sum_{k=0}^{n}\left(f \circ T^{k}-\mu_{0}(f)\right) \leq t\right]\right)=\frac{1}{\sqrt{2 \pi}} \int_{-\infty}^{t} \exp \left(-u^{2} / 2\right) d u
$$

for any $t \in \mathbb{R}$.

The proof of Corollary 7.3 is a modification of the proof of Theorem 7.2, observing that, by Theorem $6.1,\left(T^{*}, \mathscr{R}^{*}\right)$ is continued fraction mixing. For the proof of Theorem 7.2 the following maximal inequality is needed. 
Lemma 7.4. Let $\left(T^{*}, \mathscr{R}^{*}\right)$ be as in Theorem 7.2 and let $g \in L^{\infty}(q)$ be a bounded measurable function satisfying $q(g)=0$ and

$$
\xi_{n}:=\left(\int\left|g-E_{q}\left(g \mid\left(\mathscr{F}^{*}\right)_{0}^{n}\right)\right|^{2} d q\right)^{1 / 2} \leq C n^{-2-\zeta} \quad(n \geq 1)
$$

for some $C>0$. Then

$$
\sigma_{n}^{2} / n=\sigma_{n}^{2}(g) / n \rightarrow c_{g} \text { as } n \rightarrow \infty,
$$

where

$$
c_{g}=q\left(g^{2}\right)+2 \sum_{l=1}^{\infty} q\left(g g \circ\left(T^{*}\right)^{l}\right)<\infty
$$

converges absolutely, and if $c_{g} \neq 0$, then for every $\varepsilon, \eta>0$ there exist $n_{0}$ and $\delta_{0}>0$ such that for $n \geq n_{0}$ and $0 \leq N \leq \delta_{0} n$

$$
q\left(\left[\max _{0 \leq j \leq N}\left|\sum_{l=0}^{j} g \circ\left(T^{*}\right)^{l}\right| \geq \eta \sigma_{n}\right]\right) \leq \varepsilon .
$$

Proof. It is well known [24] that by the assumptions in the lemma, $\sigma_{n}^{2} \sim c_{g} n$. Since $\left(T^{*}, \mathscr{R}^{*}\right)$ is absolutely regular it is also aperiodic, hence, by Theorem $6.1,\left(T^{*}, \mathscr{R}^{*}\right)$ is continued fraction mixing with respect to $\mathscr{R}^{*}$.

Denote by

$$
\psi(n):=\sup \left\{\left|\frac{q(E \cap F)}{q(E) q(F)}-1\right|: F \in\left(\mathscr{F}^{*}\right)_{k+n}^{\infty} ; E \in\left(\mathscr{F}^{*}\right)_{0}^{k} ; k \geq 0\right\}
$$

the continued fraction mixing coefficients of $q$ with respect to $\mathscr{R}^{*}$.

Let $\varepsilon, \eta>0$ and fix $p_{0}$ so that for $p \geq p_{0}, \psi(p) \leq \psi\left(p_{0}\right)<1$. Denote

$$
S_{j}^{*}(g)=\sum_{l=0}^{j} g \circ\left(T^{*}\right)^{l} .
$$

Let $n_{0}$ and $\delta_{0}>0$ be chosen in such a way that for $n \geq n_{0}, \quad N \leq \delta_{0} n$, and $p=p(N) \sim N^{(1-\zeta) / 2}$,

$$
\begin{gathered}
C(N+2 p) p^{-2-\zeta} \leq \sigma_{N+2 p}, \\
\frac{4 N^{3} C^{2}}{\sigma_{n}^{2} \eta^{2}} p^{4+2 \zeta} \leq \varepsilon / 2, \\
\gamma_{N}:=(1-\psi(p))\left(1-\frac{128 \sigma_{N}^{2}}{\sigma_{n}^{2} \eta^{2}}\right)>0, \\
2 p\|g\|_{\infty} \leq \sigma_{n} \eta / 8,
\end{gathered}
$$

and

$$
\frac{32 \sigma_{N+2 p}^{2}}{\eta^{2} \min _{0 \leq t \leq N} \gamma_{t} \sigma_{n}^{2}} \leq \varepsilon / 2
$$

Let $n \geq n_{0}$ and $0 \leq N \leq \delta_{0} n$ be fixed. Let

$$
C_{t}=\left[\left|S_{t}^{0}(g)\right| \geq \sigma_{n} \eta / 2 ;\left|S_{j}^{0}(g)\right|<\sigma_{n} \eta / 2(0 \leq j<t)\right],
$$

where

$$
S_{t}^{0}(g)=\sum_{l=0}^{t} g_{p} \circ\left(T^{*}\right)^{l}, \quad g_{p}=E\left(g \mid\left(\mathscr{F}^{*}\right)_{0}^{p}\right) .
$$


Then

$$
\begin{aligned}
& q\left(\left[\left|S_{N+2 p}^{0}(g)\right| \geq \sigma_{n} \eta / 4\right]\right) \\
& \geq q\left(\left[\left|S_{N+2 p}^{0}(g)\right|>\sigma_{n} \eta / 4 ; \max _{0 \leq t \leq N}\left|S_{t}^{0}(g)\right|>\sigma_{n} \eta / 2\right]\right) \\
&=\sum_{t=0}^{N} q\left(C_{t} \cap\left[\left|S_{N+2 p}^{0}(g)\right|>\sigma_{n} \eta / 4\right]\right) \\
& \geq \sum_{t=0}^{N} q\left(C_{t} \cap\left[\left|S_{N+2 p}^{0}(g)-S_{t}^{0}(g)\right| \leq \frac{\sigma_{n} \eta}{4}\right]\right) \\
& \geq \sum_{t=0}^{N} q\left(C_{t} \cap\left[\left|S_{N+2 p}^{0}(g)-S_{t+2 p}^{0}(g)\right| \leq \frac{\sigma_{n} \eta}{8} ;\left|S_{t+2 p}^{0}(g)-S_{t}^{0}(g)\right| \leq \frac{\sigma_{n} \eta}{8}\right]\right) \\
&= \sum_{t=0}^{N} q\left(C_{t} \cap\left[\left|S_{N+2 p}^{0}(g)-S_{t+2 p}^{0}(g)\right| \leq \frac{\sigma_{n} \eta}{8}\right]\right) \\
& \geq(1-\psi(p))\left(1-\max _{0 \leq t \leq N} q\left(\left[\left|S_{N+2 p}^{0}(g)-S_{t+2 p}^{0}(g)\right| \geq \frac{\sigma_{n} \eta}{8}\right]\right)\right) \\
& \times q\left(\left[\max _{0 \leq t \leq N}\left|S_{t}^{0}(g)\right|>\frac{\eta \sigma_{n}}{2}\right]\right) .
\end{aligned}
$$

Since by a crude estimate

$$
E\left(S_{N+2 p}^{0}-S_{N+2 p}^{*}\right)^{2} \leq(N+2 p)^{2} E\left(g-g_{p}\right)^{2} \leq C(N+2 p)^{2} p^{-4-2 \zeta} \leq \sigma_{N+2 p}^{2}
$$

we obtain

$$
q\left(\left[\left|S_{N+2 p}^{0}(g)\right| \geq \sigma_{n} \eta / 4\right]\right) \leq \frac{16 E\left(S_{N+2 p}^{0}\right)^{2}}{\sigma_{n}^{2} \eta^{2}} \leq \frac{32 \sigma_{N+2 p}^{2}}{\sigma_{n}^{2} \eta^{2}}
$$

Moreover, since

$$
q\left(\left[\left|S_{N+2 p}^{0}(g)-S_{t+2 p}^{0}(g)\right| \geq \frac{\sigma_{n} \eta}{8}\right]\right) \leq \frac{128 \sigma_{N-t}^{2}}{\sigma_{n}^{2} \eta^{2}}
$$

for $0 \leq t \leq N$, it follows that

$$
\begin{aligned}
& q\left(\left[\max _{0 \leq j \leq N}\left|S_{j}^{0}(g)\right| \geq \sigma_{n} \eta / 2\right]\right) \\
& \quad \leq \frac{q\left(\left[\left|S_{N+2 p}^{0}(g)\right| \geq \sigma_{n} \eta / 4\right]\right)}{\left.(1-\psi(p))\left(1-\max _{0 \leq t \leq N} q\left(\left[\left|S_{N+2 p}^{0}(g)-S_{t+2 p}^{0}(g)\right| \geq \sigma_{n} \eta / 8\right)\right]\right)\right)} \\
& \quad \leq \frac{32 \sigma_{N+2 p}^{2}}{\eta^{2} \min _{0 \leq t \leq N} \gamma_{t} \sigma_{n}^{-2}} \leq \varepsilon / 2 .
\end{aligned}
$$


Finally observe that

$$
\begin{aligned}
q\left(\left[\left|\max _{0 \leq t \leq N} S_{t}^{*}(g)-\max _{0 \leq t \leq N} S_{t}^{0}(g)\right| \geq \sigma_{n} \eta / 2\right]\right) \\
\quad \leq q\left(\left\{\exists 0 \leq l \leq N \quad \ni\left|\left(g-g_{p}\right) \circ\left(T^{*}\right)^{l}\right| \geq \frac{\sigma \eta}{2 N}\right\}\right) \\
\quad \leq \sum_{l=0}^{N} q\left(\left[\left|\left(g-g_{p}\right) \circ\left(T^{*}\right)^{l}\right| \geq \frac{\sigma \eta}{2 N}\right]\right) \\
\quad \leq \sum_{l=0}^{N} \frac{4 N^{2}}{\sigma_{n}^{2} \eta^{2}} \xi_{p}^{2} \leq \frac{4 N^{3} C^{2}}{\sigma_{n}^{2} \eta^{2} p^{4+2 \zeta}} \leq \frac{\varepsilon}{2} .
\end{aligned}
$$

Whence

$$
q\left(\left[\left|\max _{0 \leq t \leq N} S_{t}^{*}(g)\right| \geq \sigma_{n} \eta\right]\right) \leq \varepsilon . \diamond
$$

Proof of Theorem 7.2. Let $0<\delta \leq \psi(1)$. Choose $\eta>0$ so that for $t \in \mathbb{R}$

$$
\frac{1}{\sqrt{2 \pi}} \int_{t}^{t+4 \eta} \exp \left(\frac{-u^{2}}{2}\right) d u<\delta \text {. }
$$

Set $g=f-\mu_{0}(f)$. Since $q$ is absolutely regular with rates as in the theorem, it is well known (see [24]) that $f^{*}$ satisfies the central limit theorem:

$$
\lim _{n \rightarrow \infty} q\left(\left[\frac{1}{\sigma_{n}} \sum_{k=0}^{n} f^{*} \circ\left(T^{*}\right)^{k} \leq t\right]\right)=\frac{1}{\sqrt{2 \pi}} \int_{-\infty}^{t} \exp \left(\frac{-u^{2}}{2}\right) d u .
$$

Let

$$
M_{n}(x)=\sum_{i=0}^{N_{n}(x)} N_{C}\left(\left(T^{*}\right)^{i} x\right)
$$

By definition, $n \leq M_{n}$ and so

$$
\left|\sum_{l=0}^{n} g \circ T^{l}-\sum_{l=0}^{M_{n}} g \circ T^{l}\right|=\left|\sum_{l=n+1}^{M_{n}} g \circ T^{l}\right| .
$$

The difference $M_{n}-n$ has possible values $0,1,2, \ldots$ If $M_{n}(x)-n=p>1$ and $l=\sum_{0 \leq i<N_{n}(x)} N_{C}\left(\left(T^{*}\right)^{i} x\right)$, then $l<n$ by definition, and $\left(T^{*}\right)^{N_{n}(x)} x=$ $T^{l} x$. Hence $N_{C}\left(T^{l} x\right)>M_{n}(x)-n=p$ and $N_{C}\left(T^{n} x\right)=N_{C}\left(T^{l} x\right)-(n-l)=p$, equivalently $T^{n} x \in\left[N_{C}=p\right]$. Hence, setting $p_{0}=\left[\eta \sigma_{n} A^{-1 / 2}\|g\|_{\infty}^{-1}\right]$,

$$
\mu\left(\left[\frac{\sqrt{A}}{\sigma_{n}}\left|\sum_{l=0}^{n} g \circ T^{l}-\sum_{l=0}^{M_{n}} g \circ T^{l}\right| \geq \eta\right]\right) \leq \sum_{p \geq p_{0}} \mu\left(N_{C}=p\right) \leq \delta,
$$

if $n$ is chosen large enough. Therefore there exists $k_{1}$ such that for $n>k \geq k_{1}$

$$
\mu\left(\left[\frac{\sqrt{A}}{\sigma_{n}}\left|\sum_{l=k+1}^{n} g \circ T^{l}-\sum_{l=M_{k}+1}^{M_{n}} g \circ T^{l}\right| \geq 2 \eta\right]\right) \leq 2 \delta .
$$

Let $n_{0}$ and $\delta_{0}$ be determined by $\delta$ and $\frac{\eta}{2 \sqrt{A}}$ as in Lemma 7.4. We may choose $k_{1}$ so large such that $k_{1} \geq 2 n_{0}, \psi\left(k_{1} A^{-1}\right)<\delta$, and, since $n^{-1} N_{n} \rightarrow A^{-1}$,

$$
\mu\left(\left[2\left|N_{k}-A^{-1} k\right| \geq \delta_{0} k ;\left(k \geq k_{1}\right)\right]\right) \leq \delta .
$$


Choose $n_{1} \geq k_{1}$ so large that for $n \geq n_{1}$,

$$
\mu\left(\left[\frac{\sqrt{A}}{\sigma_{n}}\left|\sum_{l=0}^{k_{1}} g \circ T^{l}\right| \geq \eta\right]\right) \leq \delta
$$

and, since $f^{*}$ satisfies the central limit theorem uniformly in $u \in \mathbb{R}$,

$$
\begin{aligned}
& q\left(\left[\frac{\sqrt{A}}{\sigma_{n}} \sum_{l=k_{1} A^{-1}+1}^{n A^{-1}} f^{*} \circ\left(T^{*}\right)^{l} \leq u\right]\right) \\
& \leq q\left(\left[\frac{1}{\sigma_{n A^{-1}}} \sum_{l=0}^{n A^{-1}} f^{*} \circ\left(T^{*}\right)^{l} \leq(u+\eta) \frac{\sigma_{n}}{\sqrt{A} \sigma_{n / A}}\right]\right) \\
& \left.\quad+q\left(|| \sum_{l=0}^{k_{1} A^{-1}} f^{*} \circ\left(T^{*}\right)^{l} \mid \geq \frac{\eta \sigma_{n}}{\sqrt{A}}\right]\right) \\
& \leq \frac{1}{\sqrt{2 \pi}} \int_{-\infty}^{u} \exp \left[\frac{-v^{2}}{2}\right] d v+2 \delta+\frac{A \sigma_{k_{1} A^{-1}}^{2}}{\eta^{2} \sigma_{n}^{2}} \\
& \leq \frac{1}{\sqrt{2 \pi}} \int_{-\infty}^{u} \exp \left[\frac{-v^{2}}{2}\right] d v+3 \delta .
\end{aligned}
$$

Here we used that $\sigma_{n}^{2}$ is regularly varying with index 1 by Lemma 7.4 and hence

$$
\sigma_{n} \sim \sqrt{A} \sigma_{n / A} .
$$

Next observe that by definition for $k<n$

$$
\sum_{l=M_{k}+1}^{M_{n}} g \circ T^{l}=\sum_{l=N_{k}+1}^{N_{n}} f^{*} \circ\left(T^{*}\right)^{l} .
$$

Therefore, if $n \geq n_{1}$, it follows from the above thatq

$$
\begin{aligned}
& \mu\left(\left[\frac{\sqrt{A}}{\sigma_{n}} \sum_{l=0}^{n} g \circ T^{l} \leq t\right]\right) \\
& \quad \leq \mu\left(\left[\frac{\sqrt{A}}{\sigma_{n}} \sum_{l=N_{k_{1}+1}}^{N_{n}} f^{*} \circ\left(T^{*}\right)^{l} \leq t+3 \eta\right]\right)+\mu\left(\left[\frac{\sqrt{A}}{\sigma_{n}}\left|\sum_{l=0}^{k_{1}} g \circ T^{l}\right| \geq \eta\right]\right) \\
& \quad+\mu\left(\left[\frac{\sqrt{A}}{\sigma_{n}}\left|\sum_{l=k_{1}+1}^{n} g \circ T^{l}-\sum_{l=N_{k_{1}}+1}^{N_{n}} f^{*} \circ\left(T^{*}\right)^{l}\right| \geq 2 \eta\right]\right) \\
& \quad \leq \mu\left(\left[\frac{\sqrt{A}}{\sigma_{n}} \sum_{l=N_{k_{1}}+1}^{N_{n}} f^{*} \circ\left(T^{*}\right)^{l} \leq t+3 \eta\right]\right)+3 \delta \\
& \quad \leq \mu\left(\left[2\left|N_{l}-A^{-1} l\right|<\delta_{0} l\left(l \geq k_{1}\right) ; \frac{\sqrt{A}}{\sigma_{n}} \sum_{l=N_{k_{1}}+1}^{N_{n}} f^{*} \circ\left(T^{*}\right)^{l} \leq t+3 \eta\right]\right)+4 \delta .
\end{aligned}
$$


Using the definition of $\mu$ in terms of $q$ in $\S 5$, it follows that for $n \geq n_{1}$

$$
\begin{aligned}
& \mu\left(\left[\frac{\sqrt{A}}{\sigma_{n}} \sum_{l=0}^{n}\left(f \circ T^{l}-\mu_{0}(f)\right) \leq t\right]\right) \\
& \leq \sum_{u=0}^{\infty} \sum_{j=u+1}^{\infty} q\left(\left[N_{C}=j ; 2\left|N_{l} \circ T^{u}-A^{-1} l\right|<\delta_{0} l\left(l \geq k_{1}\right) ;\right.\right. \\
& \left.\left.\frac{\sqrt{A}}{\sigma_{n}} \sum_{l=N_{k_{1}} \circ T^{u}+1}^{N_{n} \circ T^{u}} f^{*} \circ\left(T^{*}\right)^{l} \circ T^{u} \leq t+3 \eta\right]\right)+4 \delta .
\end{aligned}
$$

Note that for $j>u$ and $x \in\left[N_{C}=j\right]$ we have that $T^{*}\left(T^{u} x\right)=T^{*} x$ by definition of $T^{*}$ and that $N_{l}\left(T^{u} x\right)=N_{l}(x)$ by definition of $N_{C}$ and $N_{l}$. Therefore, using continued fraction mixing and Lemma 7.4,

$$
\begin{aligned}
& \mu\left(\left[\frac{\sqrt{A}}{\sigma_{n}} \sum_{l=0}^{n}\left(f \circ T^{l}-\mu_{0}(f)\right) \leq t\right]\right) \\
& \leq 4 \delta+\sum_{u=0}^{\infty} \sum_{j=u+1}^{\infty} q\left(\left[N_{C}=j ; \frac{\sqrt{A}}{\sigma_{n}} \sum_{l=k_{1} A^{-1}+1}^{n A^{-1}} f^{*} \circ\left(T^{*}\right)^{l} \leq t+4 \eta\right]\right) \\
& +q\left(\left[N_{C}=j ; \max _{2\left|w-k_{1} / A\right| \leq \delta_{0} k_{1}} \max _{2|v-n / A| \leq \delta_{0} n} \frac{\sqrt{A}}{\sigma_{n}}\right.\right. \\
& \left.\left.\cdot\left|\sum_{l=w+1}^{v} f^{*} \circ\left(T^{*}\right)^{l}-\sum_{l=k_{1} / A+1}^{n / A} f^{*} \circ\left(T^{*}\right)^{l}\right|>\eta\right]\right) \\
& \leq 4 \delta+\sum_{u=0}^{\infty} \sum_{j=u+1}^{\infty} q\left(\left[N_{C}=j ; \frac{\sqrt{A}}{\sigma_{n}} \sum_{l=k_{1} A^{-1}+1}^{n A^{-1}} f^{*} \circ\left(T^{*}\right)^{l} \leq t+4 \eta\right]\right) \\
& +q\left(\left[N_{C}=j ; \max _{2|v-n / A| \leq \delta_{0} n} \frac{\sqrt{A}}{\sigma_{n}}\left|\sum_{l \in[v, n / A]} f^{*} \circ\left(T^{*}\right)^{l}\right|>\eta / 2\right]\right) \\
& +q\left(\left[N_{C}=j ; \max _{2\left|w-k_{1} / A\right| \leq \delta_{0} k_{1}} \frac{\sqrt{A}}{\sigma_{n}}\left|\sum_{l \in\left[w, k_{1} / A+1\right]} f^{*} \circ\left(T^{*}\right)^{l}\right|>\eta / 2\right]\right) \\
& \leq 4 \delta+\sum_{u=0}^{\infty} \sum_{j=u+1}^{\infty} q\left(\left[N_{C}=j ; \frac{\sqrt{A}}{\sigma_{n}} \sum_{l=k_{1} A^{-1}+1}^{n A^{-1}} f^{*} \circ\left(T^{*}\right)^{l} \leq t+4 \eta\right]\right) \\
& +(1+\psi(1)) q\left(\left[N_{C}=j\right]\right) \\
& \times q\left(\left[\max _{2|v-n / A| \leq \delta_{0} n} \frac{\sqrt{A}}{\sigma_{n}}\left|\sum_{l \in[v, n / A]} f^{*} \circ\left(T^{*}\right)^{l}\right|>\eta / 2\right]\right)+(1+\psi(1)) \\
& \times q\left(\left[N_{C}=j\right]\right) q\left(\left[\max _{2\left|w-k_{1} / A\right| \leq \delta_{0} k_{1}} \frac{\sqrt{A}}{\sigma_{n}}\left|\sum_{l \in\left[w, k_{1} / A+1\right]} f^{*} \circ\left(T^{*}\right)^{l}\right|>\eta / 2\right]\right)
\end{aligned}
$$




$$
\begin{aligned}
\leq & \sum_{u=0}^{\infty} \sum_{j=u+1}^{\infty} q\left(\left[N_{C}=j ; \frac{\sqrt{A}}{\sigma_{n}} \sum_{l=k_{1} A^{-1}+1}^{n A^{-1}} f^{*} \circ\left(T^{*}\right)^{l} \leq t+4 \eta\right]\right) \\
& +(4+2 A(1+\psi(1))) \delta .
\end{aligned}
$$

Using the asymptotic normality for $f^{*}$ and the $\psi$-mixing property we obtain

$$
\begin{aligned}
& q\left(\left[N_{C}=j ; \frac{\sqrt{A}}{\sigma_{n}} \sum_{l=k_{1} A^{-1}+1}^{n A^{-1}} f^{*} \circ\left(T^{*}\right)^{l} \leq t+4 \eta\right]\right) \\
& \quad \leq\left(1+\psi\left(k_{1} A^{-1}\right)\right) q\left(\left[N_{C}=j\right]\right) q\left(\left[\frac{\sqrt{A}}{\sigma_{n}} \sum_{l=k_{1} A^{-1}+1}^{n A^{-1}} f^{*} \circ\left(T^{*}\right)^{l} \leq t+4 \eta\right]\right) \\
& \quad \leq\left(1+\psi\left(k_{1} A^{-1}\right)\right) q\left(\left[N_{C}=j\right]\right)\left(\frac{1}{\sqrt{2 \pi}} \int_{-\infty}^{t+4 \eta} \exp \left[\frac{-u^{2}}{2}\right] d u+3 \delta\right) .
\end{aligned}
$$

Consequently,

$$
\begin{aligned}
& \mu\left(\left[\frac{\sqrt{A}}{\sigma_{n}} \sum_{l=0}^{n}\left(f \circ T^{l}-\mu_{0}(f)\right) \leq t\right]\right) \\
& \leq \sum_{u=0}^{\infty} \sum_{j=u+1}^{\infty}\left(1+\psi\left(k_{1} A^{-1}\right)\right) q\left(\left[N_{C}=j\right]\right)\left(\frac{1}{\sqrt{2 \pi}} \int_{-\infty}^{t+4 \eta} \exp \left[\frac{-u^{2}}{2}\right] d u+3 \delta\right) \\
& \quad+(4+2 A(1+\psi(1))) \delta \\
& \leq \sum_{u=0}^{\infty} \sum_{j=u+1}^{\infty} q\left(\left[N_{C}=j\right]\right) \frac{1}{\sqrt{2 \pi}} \int_{-\infty}^{t} \exp \left[\frac{-u^{2}}{2}\right] d u \\
& \quad+\sum_{u=0}^{\infty} \sum_{j=u+1}^{\infty} q\left(\left[N_{C}=j\right]\right)\left[\psi\left(k_{1} A^{-1}\right)(1+3 \delta)+3 \delta\right]+(4+2 A(1+\psi(1))) \delta \\
& \leq \frac{\mu(X)}{\sqrt{2 \pi}} \int_{-\infty}^{t} \exp \left[\frac{-u^{2}}{2}\right] d u+(4+9 A(1+\psi(1))) \delta .
\end{aligned}
$$

The lower bound is proved similarly. $\diamond$

\section{CONFORMAL MEASURES FOR PARABOLIC RATIONAL MAPS}

In this section, we study parabolic rational maps. For completeness we begin with some notation. Let $T: \overline{\mathbb{C}} \rightarrow \overline{\mathbb{C}}$ be a rational map of the Riemann sphere $\overline{\mathbb{C}}$ equipped with the spherical metric $d$. We denote by $J(T)$ the Julia set of $T$. Recall that $\left.T\right|_{J(T)}$ is expansive if there exists $\beta>1$ (an expansive constant) such that

$$
\sup _{n \geq 1} d\left(T^{n}(x), T^{n}(y)\right) \geq \beta
$$

for all $x, y \in J(T), x \neq y$. This property does not depend on the metric. On the other hand, a rational map $T$ is called expanding if

$$
\exists n \geq 1 \ni \forall x \in J(T),\left|\left(T^{n}\right)^{\prime}(x)\right|>1 .
$$

Recall from [14] that a rational map $T: \overline{\mathbb{C}} \rightarrow \overline{\mathbb{C}}$ of the Riemann sphere $\overline{\mathbb{C}}$ is said to be parabolic if the restriction $\left.T\right|_{J(T)}$ is expansive but not expanding 
in the spherical metric on $\overline{\mathbb{C}}$. It is proved in [15] that $T$ is parabolic if and only if the Julia set $J(T)$ contains no critical point of $T$, but contains rationally indifferent periodic points. Denote by $\Lambda$ the set of rationally indifferent periodic points of $T$. It is known that this set is finite (see [28]).

The proof of the following elementary fact can be abstracted from [36, pp. $81,82]$.

Lemma 8.1. If $b>0$ is a real, $p \geq 1$, and $\left\{x_{n}\right\}_{n=1}^{\infty}$ is a decreasing sequence of positive reals such that

$$
\lim _{n \rightarrow \infty} x_{n}=0 \quad \text { and } \quad \lim _{n \rightarrow \infty} \frac{x_{n}-x_{n+1}}{x_{n}^{p+1}}=b,
$$

then

$$
\lim _{n \rightarrow \infty} \frac{x_{n}}{n^{-1 / p}}=(b p)^{-1 / p} \quad \text { and } \quad \lim _{n \rightarrow \infty} \frac{x_{n}-x_{n-1}}{n^{-(p+1) / p}}=b(b p)^{-(p+1) / p} .
$$

Now let $T: \overline{\mathbb{C}} \rightarrow \overline{\mathbb{C}}$ be a parabolic rational map. Using a homographical change of coordinates we can suppose that $\infty$ does not belong to the Julia set $J(T)$. In particular, $\infty$ is not a rationally indifferent periodic point of $T$.

Suppose that 0 is a rationally indifferent fixed point with $T^{\prime}(0)=1$. Then on a sufficiently small open neighbourhood $V$ of 0 a holomorphic inverse branch $T_{0}^{-1}: V \rightarrow \mathbb{C}$ of $T$ is well defined which sends 0 to 0 . Moreover, we can suppose that $V$ is so small that on $V$ the transformation $T_{0}^{-1}$ can be expressed in the form

$$
T_{0}^{-1}(z)=z-a z^{p+1}+a_{2} z^{p+2}+a_{3} z^{p+3}+\cdots,
$$

where $a \neq 0$ and $p$ is a positive integer. Consider the set $\left\{z: a z^{p} \in \mathbb{R}\right.$ and $\left.a z^{p}>0\right\}$. This set is the union of $p$ rays beginning in 0 and forming angles which are integer multiples of $\frac{2 \pi}{p}$. Denote these rays by $L_{1}, L_{2}, \ldots, L_{p}$.

For $1 \leq j \leq p, 0<r \leq \infty$, and $0 \leq \theta<2 \pi$ let $S_{j}(r, \theta) \subset V$ be the set of those points $z$ lying in the open ball $B(0, r)$ for which the angle between the rays $L_{j}$ and the interval which joins the points 0 and $z$ does not exceed $\theta$. Using the power series for $T_{0}^{-1}$ in $V$, an easy computation shows the angular stability,

$$
\begin{gathered}
\forall \theta>0 \exists r_{1}(\theta)>0 \exists 0<\theta_{0} \leq \theta \forall 1 \leq j \leq p \\
T_{0}^{-1}\left(S_{j}\left(r_{1}(\theta), \theta_{0}\right)\right) \subset S_{j}(\infty, \theta),
\end{gathered}
$$

and the contractive property that there are $\beta>0$ and $\delta_{1}>0$ such that

$$
\left|T_{0}^{-1}(z)\right|<|z|
$$

for every $0 \neq z \in S_{1}\left(\delta_{1}, \beta\right) \cup \cdots \cup S_{p}\left(\delta_{1}, \beta\right)$. The following version of Fatou's flower theorem (see $[19 ; 20 ; 8$, Theorem $3.12 ; 17, \S 3.7 ; 18$, Exposé IX]) shows that the Julia set $J(T)$ approaches the fixed point 0 tangentially to the lines $L_{1}, L_{2}, \ldots, L_{p}$. This can be precisely formulated as follows.

Lemma 8.2 (Fatou's flower theorem). For every $\theta>0$ there exists $r_{2}(\theta)>0$ such that

$$
J(T) \cap B\left(0, r_{2}(\theta)\right) \subset S_{1}\left(r_{2}(\theta), \theta\right) \cup \cdots \cup S_{p}\left(r_{2}(\theta), \theta\right) .
$$


Since the Julia set $J(T)$ is fully invariant $\left(T^{-1}(J(T))=J(T)=T(J(T))\right)$, we conclude from this lemma and the contractive property that for $0<\delta_{2}=$ $\min \left\{\delta_{1}, r_{2}(\beta)\right\}$ we have

$$
T_{0}^{-1}\left(J(T) \cap B\left(0, \delta_{2}\right)\right) \subset J(T) \cap B\left(0, \delta_{2}\right) .
$$

Thus all iterations $T_{0}^{-n}: J(T) \cap B\left(0, \delta_{2}\right) \rightarrow J(T) \cap B\left(0, \delta_{2}\right), n=0,1,2, \ldots$, are well defined. From angular stability, Lemma 8.2, and the contractive property we obtain a contractive angular stability:

$$
\begin{gathered}
\forall \theta>0 \exists r_{3}(\theta)>0 \forall 1 \leq j \leq p \\
T_{0}^{-1}\left(S_{j}\left(r_{3}(\theta), \theta\right) \cap J(T)\right) \subset S_{j}\left(r_{3}(\theta), \theta\right) .
\end{gathered}
$$

Put $\delta=\min \left\{\delta_{2}, r_{3}(\beta)\right\}$. Fix $z \in J(T) \cap B(0, \delta)$ and for every $n \geq 0$ let

$$
z_{n}=T_{0}^{-n}(z) \text {. }
$$

It follows from the contractive property, contractive angular stability, and Lemma 8.2 that

$$
\lim _{n \rightarrow \infty} z_{n}=0
$$

and therefore, in view of Lemma 8.2, a simple computation based on the power series for $T_{0}^{-1}$ proves that

$$
\lim _{n \rightarrow \infty} \frac{\left|z_{n}-z_{n+1}\right|}{\left|z_{n}\right|-\left|z_{n+1}\right|}=1 \text {. }
$$

Hence, again by the power series for $T_{0}^{-1}$,

$$
\lim _{n \rightarrow \infty} \frac{\left|z_{n}\right|-\left|z_{n+1}\right|}{\left|z_{n}\right|^{p+1}}=|a| \text {. }
$$

In view of this and the contractive property, we conclude that Lemma 8.1 applies to the sequence $x_{n}=\left|z_{n}\right|$. Thus,

$$
\lim _{n \rightarrow \infty} \frac{\left|z_{n}\right|}{n^{-1 / p}}=(|a| p)^{-1 / p}
$$

and

Combining all this, we get

$$
\lim _{n \rightarrow \infty} \frac{\left|z_{n}\right|-\left|z_{n+1}\right|}{n^{-(p+1) / p}}=|a|(|a| p)^{-(p+1) / p} .
$$

$$
\lim _{n \rightarrow \infty} \frac{\left|z_{n}-z_{n+1}\right|}{n^{-(p+1) / p}}=|a|(|a| p)^{-(p+1) / p} .
$$

Now let $\omega \in \mathbb{C}$ be an arbitrary rationally indifferent fixed point of $T$ with $T^{\prime}(\omega)=1$ and let $V_{\omega}^{\prime} \subset \mathbb{C}$ be an open neighbourhood of $\omega$ of diameter not exceeding an expansive constant for $T: J(T) \rightarrow J(T)$ and with the following properties: There exists a unique holomorphic inverse branch $T_{\omega}^{-1}$ of $T$ defined on $V_{\omega}^{\prime}$ which sends $\omega$ to $\omega$. Moreover we suppose $V_{\omega}^{\prime}$ to be so small that

$$
T_{\omega}^{-1}\left(V_{\omega}^{\prime} \cap J(T)\right) \subset V_{\omega}^{\prime} \cap J(T)
$$

and that $T_{\omega}^{-1}$ is given on $V_{\omega}^{\prime}$ by

$$
T_{\omega}^{-1}(z)-\omega=z-\omega-a(z-\omega)^{p+1}+a_{2}(z-\omega)^{p+2}+a_{3}(z-\omega)^{p+3}+\cdots,
$$

where $0 \neq a \in \mathbb{C}$ and $p=p(\omega)$ is a positive integer (sometimes we write $p(T, \omega)$ in order to indicate the map under consideration).

Using the translation that maps $\omega$ to 0 as a change of coordinates, we obtain 
Proposition 8.3. If $T: \overline{\mathbb{C}} \rightarrow \overline{\mathbb{C}}$ is a parabolic rational map and $\omega \in \mathbb{C}$ is a rationally indifferent fixed point of $T$ with $T^{\prime}(\omega)=1$, then there exists an open neighbourhood $V_{\omega} \subset V_{\omega}^{\prime}$ of $\omega$ such that for every $z \in V_{\omega} \cap J(T)$ we have

$$
\lim _{n \rightarrow \infty} \frac{\left|z_{n}-\omega\right|}{n^{-1 / p}}=(|a| p)^{-1 / p}
$$

and

$$
\lim _{n \rightarrow \infty} \frac{\left|z_{n}-z_{n+1}\right|}{n^{-(p+1) / p}}=|a|(|a| p)^{-(p+1) / p},
$$

where $z_{n}=T_{\omega}^{-n} z$.

Combining Proposition 8.3 and the Köbe distortion theorem (see [22]) we obtain the next result.

We shall prove the following.

Theorem 8.4. Let $\omega \in \Lambda$ be a fixed point with $T^{\prime} \omega=1$. For any $\omega \neq z \in V_{\omega}$ define

$$
\underline{g}(z)=\liminf _{n \rightarrow \infty}|z-\omega|^{p(\omega)+1} \frac{n^{(p(\omega)+1) / p(\omega)}}{\left|\left(T^{n}\right)^{\prime} z_{n}\right|}
$$

and

$$
\bar{g}(z)=\limsup _{n \rightarrow \infty}|z-\omega|^{p(\omega)+1} \frac{n^{(p(\omega)+1) / p(\omega)}}{\left|\left(T^{n}\right)^{\prime} z_{n}\right|},
$$

then

$$
\lim _{z \rightarrow \omega} \underline{g}(z)=\lim _{z \rightarrow \omega} \bar{g}(z)=(|a| p(\omega))^{-(p(\omega)+1) / p(\omega)} .
$$

In particular, there is a constant $C(z) \in \mathbb{R}_{+}$such that

$$
C(z)^{-1} n^{\frac{p(\omega)+1}{p(\omega)}} \leq\left|\left(T^{n}\right)^{\prime} z_{n}\right| \leq C(z) n^{\frac{p(\omega)+1}{p(\omega)}} .
$$

Proof. Put $p=p(\omega)$. Without loss of generality we can suppose that $\omega=0$. Since $J(T) \cap \operatorname{Crit}(T)=\varnothing$, it follows from the flower theorem that for every $0<\theta<\pi / p, \exists r_{3}(\theta)>0$ such that

$$
\bigcup_{n=0}^{\infty} T^{n}(\operatorname{Crit}(T)) \cap\left(S_{1}\left(r_{3}(\theta), \theta\right) \cup \cdots \cup S_{p}\left(r_{3}(\theta), \theta\right)\right)=\varnothing .
$$

For every $0 \neq z \in \overline{\mathbb{C}}$ let

$$
D(z)=B\left(z, \frac{1}{2}|z| \cos \frac{\pi}{2 p}\right) .
$$

By the flower theorem there exists $r_{4}>0$ such that if $z \in J(T)$ and $|z| \leq r_{4}$ then

$$
D(z) \subset S_{1}\left(r_{3}\left(\frac{\pi}{2 p}\right), \frac{\pi}{2 p}\right) \cup \cdots \cup S_{p}\left(r_{3}\left(\frac{\pi}{2 p}\right), \frac{\pi}{2 p}\right)
$$

and therefore all holomorphic inverse branches of all iterations $T^{n}, n=0,1$, $2, \ldots$, are well defined on $D(z)$. In particular, we denote by $T_{z}^{-n}: D(z) \rightarrow \mathbb{C}$ the inverse branch of $T^{n}$ which sends $z$ to $z_{n}$. It follows from the power series for $T_{0}^{-1}$ that there exists $0<r_{5} \leq r_{4}$ such that if $|z| \leq r_{5}$, then $z_{1} \in$ $B\left(z, \frac{1}{4}|z| \cos \frac{\pi}{2 p}\right)$. Thus, by the Köbe distortion theorem and the definition of $D(z)$, we get for every $n \geq 1$

$$
(t(z))^{-1}\left|z_{n}-z_{n+1}\right| \leq\left|\left(T_{z}^{-n}\right)^{\prime}(z)\right|\left|z-z_{1}\right| \leq t(z)\left|z_{n}-z_{n+1}\right|,
$$


where $t(z)=k\left(2\left|z_{1}-z\right| /|z| \cos \pi / 2 p\right)$ and $k$ is the function appearing in the Köbe distortion theorem. Hence, in view of Proposition 8.3, we have

$$
(t(z))^{-1} \frac{|z|^{p+1}}{\left|z-z_{1}\right|}|a|(|a| p)^{-\frac{p+1}{p}} \leq \underline{g}(z) \leq \bar{g}(z) \leq t(z) \frac{|z|^{p+1}}{\left|z-z_{1}\right|}|a|(|a| p)^{-\frac{p+1}{p}} .
$$

The theorem follows from the power series for $T_{0}^{-1}, \lim _{z \rightarrow \omega} t(z)=1$ and $\lim _{z \rightarrow \omega}\left(|z|^{p+1} /\left|z-z_{1}\right|\right)=1 /|a| . \diamond$

Next, we extend the definition of $p(\omega)$ given by the power series of $T_{\omega}^{-1}$ to the set $\Lambda$ of all rationally indifferent perodic points $\omega$ of $T$ putting $p(\omega)=$ $p\left(T^{q}, \omega\right)$, where $q \geq 1$ is taken so that $T^{q}(\omega)=\omega$ and $\left(T^{q}\right)^{\prime}(\omega)=1$. Note that $p(\omega)$ does not depend on $q$ with these properties. We shall prove the following.

Theorem 8.5. If $T: \overline{\mathbb{C}} \rightarrow \overline{\mathbb{C}}$ is a parabolic rational map, then $h=H D(J(T))>$ $\max \{p(\omega) / p(\omega)+1: \omega \in \Lambda\}$.

Proof. Since $J\left(T^{n}\right)=J(T)$ for every $n \geq 1$, we can suppose that $\Lambda$ consists only of rationally indifferent fixed points $\omega$ with $T^{\prime}(\omega)=1$. Take an arbitrary point $\omega \neq z \in V_{\omega} \cap J(T)$ and consider $r>0$ so small that the ball $B(z, 2 r)$ contains no iteration of a critical value of $T$. Therefore (compare the proof of Theorem 8.4) for every $n \geq 1$ there exists a unique holomorphic inverse branch $T_{z}^{-n}: B(z, 2 r) \rightarrow \mathbb{C}$ of $T^{n}$ defined on $B(z, 2 r)$ and determined by the condition $T_{z}^{-n}(z)=z_{n}$. The radius $r$ is also assumed to be small enough such that $T_{z}^{-1}(B(z, 2 r)) \cap B(z, 2 r)=\varnothing$. Then for all positive integers $k \neq l$

$$
T_{z}^{-k}(B(z, 2 r)) \cap T_{z}^{-l}(B(z, 2 r))=\varnothing .
$$

By [15, Theorem 13(iii)] there exists an $h$-conformal measure $m$ for $T$ : $J(T) \rightarrow J(T)$. In view of this, and the Köbe distortion theorem it follows from conformality of the measure $m$ that

$$
1 \geq \sum_{n=1}^{\infty} m\left(T_{z}^{-n}(B(z, r))\right) \geq K^{-h} m(B(z, r)) \sum_{n=1}^{\infty}\left|\left(T_{z}^{-n}\right)^{\prime}(z)\right|^{h},
$$

where $K$ is the constant appearing in the Köbe distortion theorem. Since $m(B(z, r))>0$, this formula implies that the series $\sum_{n=1}^{\infty}\left|\left(T_{z}^{-n}\right)^{\prime}(z)\right|^{h}$ converges. Therefore, as

$$
\left|\left(T_{z}^{-n}\right)^{\prime}(z)\right|^{h}=\left|\left(T^{n}\right)^{\prime}\left(z_{n}\right)\right|^{-h},
$$

it follows from Theorem 8.4 that $h>p(\omega) /(p(\omega)+1)$. The proof is finished. $\diamond$

Corollary 8.6. If $T: \overline{\mathbb{C}} \rightarrow \overline{\mathbb{C}}$ is a parabolic rational map then $H D(J(T))>1 / 2$.

We now prove the main result in this section.

Theorem 8.7. If $T: \overline{\mathbb{C}} \rightarrow \overline{\mathbb{C}}$ is a parabolic rational map then there exists exactly one h-conformal measure for $T$. Moreover this measure is nonatomic.

Proof. By [15, Theorem 13(iii)], there is an $h$-conformal measure for $T$. By [14, Theorem 4.6], this measure, if nonatomic, is unique. Since $J\left(T^{n}\right)=J(T)$ for every $n \geq 1$ and since any $t$-conformal measure for $T$ is also $t$-conformal for $T^{n}$, the existence of a nonatomic $h$-conformal measure for $T^{n}$ for some 
$n \geq 1$ will establish the theorem. Consequently, we can suppose that all rationally indifferent periodic points of $T$ are fixed points and their derivatives are equal to 1 .

From Theorems 8.4 and 8.5 we deduce that there exists a constant $\sigma>0$ such that for every rationally indifferent fixed point $\omega$ and every point $\omega \neq z \in V_{\omega}$

$$
\begin{aligned}
& \exists(C(\omega, z) \geq 1) \forall(t \geq h-\sigma) \forall(k \geq 1) \\
& \sum_{n=k}^{\infty}\left|\left(T^{n}\right)^{\prime}\left(z_{n}\right)\right|^{-t} \leq C(\omega, z) \sum_{n=k}^{\infty} \frac{1}{n^{1+\sigma}} .
\end{aligned}
$$

Now let $W_{n}=B\left(\Lambda, \frac{1}{n}\right), n=1,2, \ldots$, and let

$$
K_{n}=\left\{z \in J(T): T^{k}(z) \notin W_{n} \text { for every } k \geq 0\right\} .
$$

The sets $K_{n}$ are closed and forward invariant under $T$. Since $J(T)$ contains no critical points of $T$, we obtain from $[16, \S 5]$, an increasing sequence $\left\{t_{n}\right\} \subset$ $(0, h]$, and probability measures $m_{n} \in \mathscr{P}\left(K_{n}\right) \quad(n \geq 1)$ with the properties

$$
m_{n}(T(A)) \geq \int_{A}\left|T^{\prime}\right|^{t_{n}} d m_{n}
$$

for every special set $A \subset J(T)$ and

$$
m_{n}(T(A))=\int_{A}\left|T^{\prime}\right|^{t_{n}} d m_{n}
$$

for every special set $A \subset J(T)$ disjoint from $\overline{W_{n}}$. (A set $A \in \mathscr{F}$ is called special if $T: A \rightarrow T(A)$ is invertible.)

Let $m$ be an arbitrary weak accumulation point of the sequence $\left\{m_{n}\right\}_{n=1}^{\infty} \subset$ $\mathscr{P}(J(T))$. Fix $k \geq 1$. Since $T: J(T) \rightarrow J(T)$ is an open map, and since $J(T)$ contains no critical points of $T$, it follows from [16, Lemma 3.3] that

$$
m(T(A))=\int_{A}\left|T^{\prime}\right|^{u} d m
$$

for every special set $A \subset J(T)$ disjoint from $\overline{W_{k}}$, where $t_{n} \rightarrow u$. Therefore letting $k \rightarrow \infty$ we conclude that this is true for every special set $A \subset J(T)$ disjoint from $\Lambda$ and, since $\left|T^{\prime}(\omega)\right|=1$ for every $\omega \in \Lambda$, for every special set $A \subset J(T)$. Consequently $m$ is a $u$-conformal measure for $T: J(T) \rightarrow J(T)$. As $u \leq h$, it follows from [15, Theorem 14] that $u=h$.

In view of $[15$, Lemma $6(i i)]$, it is sufficient to show that $m(\Lambda)=0$. To this end fix $\omega \in \Lambda$ and consider the ring

$$
R(\omega)=\left\{z \in \mathbb{C}: r\left\|T^{\prime}\right\|^{-1} \leq|z-\omega| \leq r\right\},
$$

where $\left\|T^{\prime}\right\|=\sup \left\{\left|T^{\prime}(z)\right|: z \in J(T)\right\}>1$ and where $r>0$ is so small that

$$
\overline{B(\omega, r)} \subset V_{\omega} \text {. }
$$

Since $J(T)$ contains no critical points of $T$, since the $\omega$-limit set of critical points of $T$ intersects the Julia set $J(T)$ only on $\Lambda$, and $R(\omega) \cap J(T)$ is compact, we can find finitely many points, say, $x_{1}, \ldots, x_{q} \in R(\omega) \cap J(T)$, and positive reals $r_{1}, \ldots, r_{q}$ such that

$R(\omega) \cap J(T) \subset B\left(x_{1}, r_{1}\right) \cup \cdots \cup B\left(x_{q}, r_{q}\right) \subset B\left(x_{1}, 2 r_{1}\right) \cup \cdots \cup B\left(x_{q}, 2 r_{q}\right) \subset V_{\omega}$ 
and for every $i=1, \ldots, q$

$$
B\left(x_{i}, 2 r_{i}\right) \cap \bigcup_{n=0}^{\infty} T^{n}(\operatorname{Crit}(T))=\varnothing .
$$

Hence for every $i=1, \ldots, q$ and every $n \geq 1$ there exists a unique holomorphic inverse branch $T_{i}^{-n}: B\left(x_{i}, 2 r_{i}\right) \rightarrow \mathbb{C}$ of $T^{n}$ such that $T_{i}^{-n}\left(x_{i}\right)=T_{\omega}^{-n}\left(x_{i}\right)$.

Fix a neighbourhood $V \subset V_{\omega}$ of $\omega$. Since $T: J(T) \rightarrow J(T)$ is expansive and $r \leq \operatorname{diam}\left(V_{\omega}\right)$ does not exceed an expansive constant of $T: J(T) \rightarrow J(T)$, we conclude that for every $\omega \neq x \in B(\omega, r) \cap J(T)$ there exists $n \geq 0$ such that $T^{n}(x) \notin B\left(\omega,\left\|T^{\prime}\right\|^{-1} r\right)$. If we denote by $n(x)$ the smallest integer with this property, then $T^{n(x)} \in R(\omega)$. Thus by the above,

$$
V \cap J(T) \subset\{\omega\} \cup \bigcup_{i=1}^{q} \bigcup_{n=n(V)}^{\infty} T_{i}^{-n}\left(B\left(x_{i}, r_{i}\right)\right),
$$

where $n(V)=\min \{n(x): \omega \neq x \in V \cap J(T)\}$. Therefore using the properties of $\left\{m_{n}\right\}$ and the Köbe distortion theorem we obtain for every $k \geq 1$

$$
\begin{aligned}
m_{k}(V \cap J(T)) & \leq K^{t_{k}} \sum_{i=1}^{q} \sum_{n=n(V)}^{\infty}\left|\left(T_{i}^{-n}\right)^{\prime}\left(x_{i}\right)\right|^{t_{k}} m_{k}\left(B\left(x_{i}, r_{i}\right)\right) \\
& \leq K^{t_{k}} \sum_{i=1}^{q} \sum_{n=n(V)}^{\infty}\left|\left(T_{i}^{-n}\right)^{\prime}\left(x_{i}\right)\right|^{t_{k}}
\end{aligned}
$$

where $K$ is the constant appearing in the Köbe distortion theorem. Hence, putting $C=K^{h} \max \left\{C\left(\omega, x_{i}\right): i=1, \ldots q\right\}$, it follows that for every $k$ so big that $t_{k} \geq h-\sigma$ we have

$$
m_{k}(V \cap J(T)) \leq C \sum_{n=n(V)}^{\infty} \frac{1}{n^{1+\sigma}} .
$$

Thus, letting $k \rightarrow \infty$

$$
m(V \cap J(T)) \leq C \sum_{n=n(V)}^{\infty} \frac{1}{n^{1+\sigma}} .
$$

This proves that $m(\omega)=0$, since $n(V) \rightarrow \infty$ as $V \downarrow \omega$. $\diamond$

The following is a strengthening of [15, Theorem 17], that the (2-dimensional) Lebesgue measure of $J(T)$ is equal to zero.

Theorem 8.8. If $T: \overline{\mathbb{C}} \rightarrow \overline{\mathbb{C}}$ is a parabolic rational map then $h=H D(J(T))<$ 2 .

Proof. Suppose otherwise that $h=2$ and let $m$ be the 2-conformal measure in Theorem 8.7. Put $X=J(T) \backslash \bigcup_{n=0}^{\infty} T^{-n}(\Lambda)$. By Lemma 10 in [15], there exists a constant $B \geq 1$ and for every $x \in X$ there exists a sequence $\left\{r_{j}(x)\right\}_{j=1}^{\infty}$ of positive reals decreasing to 0 such that

$$
B^{-1} \leq \frac{m\left(B\left(x, r_{j}(x)\right)\right)}{r_{j}(x)^{2}} \leq B
$$


Fix $\varepsilon>0$ and denote by $\lambda$ the (2-dimensional) Lebesgue measure on $\mathbb{C}$. Since $\lambda(J(T))=0$ by [15, Theorem 17], and since $\lim _{j \rightarrow \infty} r_{j}(x)=0$ for every $x \in X$, there exists a radius $r(x)$ being of the form $r_{j}(x)$ such that

$$
\lambda\left(\bigcup_{x \in X} B(x, r(x))\right)<\varepsilon .
$$

Now by the Besicovich covering theorem (see [21]) we can choose a countable subcover $\left\{B\left(x_{i}, r\left(x_{i}\right)\right)\right\}_{i=1}^{\infty}$ of the cover $\{B(x, r(x))\}_{x \in X}$ of $X$, of multipicity bounded by some universal constant $C \geq 1$. Therefore we obtain

$$
\begin{aligned}
m(X) & \leq \sum_{i=1}^{\infty} m\left(B\left(x_{i}, r\left(x_{i}\right)\right)\right) \leq B \sum_{i=1}^{\infty} r\left(x_{i}\right)^{2}=B \pi^{-1} \sum_{i=1}^{\infty} \lambda\left(B\left(x_{i}, r\left(x_{i}\right)\right)\right) \\
& \leq B \pi^{-1} C \lambda\left(\bigcup_{i=1}^{\infty} B\left(x_{i}, r\left(x_{i}\right)\right)\right) \leq B C \pi^{-1} \varepsilon .
\end{aligned}
$$

Thus, letting $\varepsilon \rightarrow 0$, we obtain $m(X)=0$. Since $m$ is nonatomic, it implies that $m(J(T))=0$. This contradiction finishes the proof. $\diamond$

The next lemma will be used in the sequel.

Lemma 8.9. If $E \subset V_{\omega} \cap B\left(\omega, r_{5}\right) \cap J(T)$ is a Borel set and $\omega \notin \bar{E}$, then there exists a constant $c(E)$ such that

$$
\lim _{n \rightarrow \infty} m\left(T_{\omega}^{-n}(E)\right) n^{\frac{p(\omega)+1}{p(\omega)} h}=c(E),
$$

where $r_{5}$ is as defined in the proof of Theorem 8.4.

Proof. Put again $p=p(\omega)$ and also $\alpha=\frac{p+1}{p} h$. Let $r_{6}=\inf \left\{\frac{1}{2}|z| \cos \frac{\pi}{2 p}: z \in\right.$ $E$ \}. Since $\omega \notin \bar{E}$, we see that $r_{6}>0$. We shall prove the following claim: $\forall \sigma>1 \exists r_{7}>0$ such that:

$$
\begin{gathered}
\text { If } G \subset J(T), G \cap E \neq \varnothing, \text { and } \operatorname{diam}(G) \leq r_{7} \\
\text { then } \exists q_{0}=q_{0}(\sigma, G) \text { such that } \forall q \geq q_{0} \\
\begin{aligned}
\sigma^{-1} q^{\alpha} m\left(T_{\omega}^{-q}(G)\right) & \leq \liminf _{n \rightarrow \infty} m\left(T_{\omega}^{-n}(G)\right) n^{\alpha} \\
& \leq \limsup _{n \rightarrow \infty} m\left(T_{\omega}^{-n}(G)\right) n^{\alpha} \leq \sigma q^{\alpha} m\left(T_{\omega}^{-q}(G)\right) .
\end{aligned}
\end{gathered}
$$

Take $0<r_{7}<r_{6}$ so small that $k^{2 h}\left(r_{7} / r_{6}\right)<\sqrt{\sigma}$ (k denotes the function appearing in the Köbe distortion theorem) and fix $z \in G$. For any $n \geq 0$ put $G_{n}=T_{\omega}^{-n}(G)$. Since $T_{\omega}^{-n}\left(G_{q}\right)=T_{z_{q}}^{-n}\left(G_{q}\right)$ for all $n, q \geq 0$, it follows from the Köbe distortion theorem that

$$
\begin{aligned}
(\sqrt{\sigma})^{-1} m\left(G_{q}\right) \liminf _{n \rightarrow \infty} \frac{\left|\left(T_{z_{q}}^{-n}\right)^{\prime}\right|^{h}}{n^{-\alpha}} & \leq \liminf _{n \rightarrow \infty} m\left(T_{\omega}^{-n}\left(G_{q}\right)\right) n^{\alpha} \\
& \leq \limsup _{n \rightarrow \infty} m\left(T_{\omega}^{-n}\left(G_{q}\right)\right) n^{\alpha} \leq \sqrt{\sigma} m\left(G_{q}\right) \limsup _{n \rightarrow \infty} \frac{\left|\left(T_{z_{q}}^{-n}\right)^{\prime}\right|^{h}}{n^{-\alpha}}
\end{aligned}
$$


for every $q \geq 1$. In view of Theorem 8.4 and Proposition 8.3 there exists $q_{0}$ so large that for every $q \geq q_{0}$

$$
\begin{aligned}
(\sqrt{\sigma})^{-1}(|a| p)^{-\alpha}(|a| p)^{\alpha} q^{\alpha} & \leq \liminf _{n \rightarrow \infty} \frac{\left|\left(T_{z_{q}}^{-n}\right)^{\prime}\right|^{h}}{n^{-\alpha}} \\
& \leq \limsup _{n \rightarrow \infty} \frac{\left|\left(T_{z_{q}}^{-n}\right)^{\prime}\right|^{h}}{n^{-\alpha}} \leq \sqrt{\sigma}(|a| p)^{-\alpha}(|a| p)^{\alpha} q^{\alpha} .
\end{aligned}
$$

Therefore we get for every $q \geq q_{0}$

$$
\begin{aligned}
\sigma^{-1} q^{\alpha} m\left(G_{q}\right) & \leq \liminf _{n \rightarrow \infty} m\left(T_{\omega}^{-n}\left(G_{q}\right)\right) n^{\alpha} \\
& \leq \limsup _{n \rightarrow \infty} m\left(T_{\omega}^{-n}\left(G_{q}\right)\right) n^{\alpha} \leq \sigma q^{\alpha} m\left(G_{q}\right) .
\end{aligned}
$$

Therefore the observations that $T_{\omega}^{-n}\left(G_{q}\right)=T_{\omega}^{-(n+q)}(G)$ and $\lim _{n \rightarrow \infty}\left(\frac{n+q}{n}\right)^{\alpha}=1$ finish the proof of the claim.

Now fix $\sigma>1$ and partition $E$ into finitely many mutually disjoint Borel sets $E_{1}, \ldots, E_{s}$ with diameters not exceeding $r_{\gamma}$. It follows from the claim that, setting

we obtain

$$
q=\max \left\{q_{0}\left(\sigma, E_{1}\right), \ldots, q_{0}\left(\sigma, E_{s}\right)\right\}
$$

$$
\begin{aligned}
\liminf _{n \rightarrow \infty} m\left(T_{\omega}^{-n}(E)\right) n^{\alpha} & \geq \liminf _{n \rightarrow \infty} m\left(T_{\omega}^{-n}\left(E_{1}\right)\right) n^{\alpha}+\cdots+\liminf _{n \rightarrow \infty} m\left(T_{\omega}^{-n}\left(E_{s}\right)\right) n^{\alpha} \\
& \geq \sigma^{-1} q^{\alpha}\left(m\left(T_{\omega}^{-q}\left(E_{1}\right)\right)+\cdots+m\left(T_{\omega}^{-q}\left(E_{s}\right)\right)\right) \\
& =\sigma^{-1} q^{\alpha} m\left(T_{\omega}^{-q}(E)\right)
\end{aligned}
$$

and

$$
\begin{aligned}
\limsup _{n \rightarrow \infty} m\left(T_{\omega}^{-n}(E)\right) n^{\alpha} & \leq \limsup _{n \rightarrow \infty} m\left(T_{\omega}^{-n}\left(E_{1}\right)\right) n^{\alpha}+\cdots+\underset{n \rightarrow \infty}{\limsup } m\left(T_{\omega}^{-n}\left(E_{s}\right)\right) n^{\alpha} \\
& \leq \sigma q^{\alpha}\left(m\left(T_{\omega}^{-q}\left(E_{1}\right)\right)+\cdots+m\left(T_{\omega}^{-q}\left(E_{s}\right)\right)\right) \\
& =\sigma q^{\alpha} m\left(T_{\omega}^{-q}(E)\right) .
\end{aligned}
$$

Consequently,

$$
1 \leq \frac{\limsup _{n \rightarrow \infty} m\left(T_{\omega}^{-n}(E)\right) n^{\alpha}}{\liminf \inf _{n \rightarrow \infty} m\left(T_{\omega}^{-n}(E)\right) n^{\alpha}} \leq \sigma^{2} .
$$

Therefore, letting $\sigma \searrow 1$, the lemma follows. $\diamond$

\section{PARABOLIC RATIONAL MAPS AS PARABOLIC FIBRED SYSTEMS}

Let $T: \overline{\mathbb{C}} \rightarrow \overline{\mathbb{C}}$ be a parabolic rational map of the Riemann sphere $\overline{\mathbb{C}}$ equipped with the spherical metric $d$. Denote by $\Lambda$ the set (not empty, finite) of rationally indifferent periodic points of $T$.

By Theorem 8.7 there is a nonatomic $h$-conformal measure $m$ on $J(T)$. We consider $T$ to be a nonsingular transformation of the probability space $(J(T), \mathscr{F}, m)$, where $\mathscr{F}$ denotes the $\sigma$-algebra of Borel subsets of $J(T)$. The first result of this section is

Theorem 9.1. There is a partition $\mathscr{R}$ for $T$ such that $(T, \mathscr{R})$ is a finite, aperiodic, parabolic fibred system with aperiodic jump transformation.

The proof is by construction of a suitable topological Markov partition and is in a sequence of lemmas. 
A topological Markov partition $\mathscr{A}$ for a continuous map $S$ on a topological space $X$ is a finite collection of closed sets $\{B\}$ such that

$$
\begin{aligned}
& \bigcup_{B \in \mathscr{A}} B=X ; B=\overline{B^{o}}, \\
& \forall B \in \mathscr{A} \exists \mathscr{A}_{B} \subset \mathscr{A} \ni \quad S B=\bigcup_{A \in \mathscr{A}_{B}} A, \\
& \left\{B^{o}: B \in \mathscr{A}\right\} \text { are disjoint. }
\end{aligned}
$$

One of the additional properties will be that $\left.S\right|_{B}$ is one-to-one for every $B \in \mathscr{A}$. It follows from this that necessarily

$$
S(\partial B) \subseteq \bigcup_{A \in \mathscr{A}} \partial A \quad \forall B \in \mathscr{A}
$$

It has been shown in [14] that every open expansive map (in particular $T$ : $J(T) \rightarrow J(T)$ ) admits a metric $\rho$ generating the same topology, so that the map is expanding in the sense of Ruelle [31] with respect to $\rho$, that is,

$$
\exists \eta>0 \text { and } \lambda<1 \ni \forall x, y^{\prime} \in J(T), \rho\left(T(x), y^{\prime}\right)<\eta \exists ! y \in J(T) \ni T(y)=y^{\prime}
$$

and

$$
\rho(x, y)<\lambda \rho\left(T(x), y^{\prime}\right) .
$$

Consequently (see [31, 14]) such a map admits Markov partitions of arbitrarily small diameter. Since the metrics $\rho$ and $d$ induce the same topology, the relevant results in $\S 4$ in [14] can be reformulated in the following lemma.

Lemma 9.2 (Construction). Let $\beta>0$ be an expansive constant, so small that for every pair of rationally indifferent periodic points $\omega \neq \omega^{\prime} \in J(T)$,

$$
\begin{gathered}
\operatorname{dist}(T(B(\omega, 2 \beta)), \Lambda \backslash T(\{\omega\}))>2 \beta, \\
\operatorname{dist}\left(B(\omega, 2 \beta), B\left(\omega^{\prime}, 2 \beta\right)\right)>0 .
\end{gathered}
$$

Then there exist $0<\delta<\beta$ and a Markov partition $\mathscr{A}=\left\{A_{1}, \ldots, A_{s}\right\}$ such that

(1) $\operatorname{diam} T\left(A_{k}\right)<\delta$ for $1 \leq k \leq s$. In particular, $\left.T\right|_{B}$ is 1-1 for every $B \in \mathscr{A}$.

(2) If $T\left(A_{k}\right) \cap(J(T) \backslash B(\Lambda, \beta)) \neq \varnothing$, then all inverse branches $T_{\nu}^{-n}$ of $T^{n}: \overline{\mathbb{C}} \rightarrow \overline{\mathbb{C}}$ are well defined and analytic on $B\left(T\left(A_{k}\right), 2 \delta\right)$ for every $n \geq 1$.

(3) If $\bigcap_{1 \leq j \leq n} T^{-j+1}\left(\operatorname{Int}\left(A_{k_{j}}\right)\right) \neq \varnothing$ and $T\left(A_{k_{n}}\right) \cap(J(T) \backslash B(\Lambda, \beta)) \neq \varnothing$, then there exists a unique analytic inverse branch $T_{\nu}^{-n}: B\left(T\left(A_{k_{n}}\right), 2 \delta\right) \rightarrow \overline{\mathbb{C}}$ of $T^{n}$ such that

$$
T_{\nu}^{-n}\left(T\left(A_{k_{n}}\right)\right)=\bigcup_{j=1}^{n} T^{-j+1}\left(A_{k_{j}}\right) .
$$

(4) $m(\partial \mathscr{A})=0$.

(5) $\max \left\{\operatorname{diam} B: B \in \mathscr{R}_{0}^{n}\right\} \rightarrow 0$ as $n \rightarrow \infty$. 
Lemma 9.3. Without loss of generality there are two (repulsive) periodic points with relatively prime periods whose orbits are outside of $B(\Lambda, \beta)$ and in the interior of sets in $\mathscr{A}$.

Proof. Since the repulsive periodic points are dense in $J(T)$ there exist two (repulsive) periodic points with relatively prime periods whose orbits are contained in the union of the interiors of the sets $A \in \mathscr{A}$. Refining the Markov partition and taking a smaller neighbourhood $B(\Lambda, \beta)$ proves the lemma.

Lemma 9.4 [14]. (1) Let

$$
X=J(T) \backslash \bigcup_{n=0}^{\infty} T^{-n}(\partial \mathscr{A})
$$

and $\mathscr{R}=\{b=A \cap X: A \in \mathscr{A}\}$. Then $(T, \mathscr{R})$ is an aperiodic Markov fibred system on $\left(X, \mathscr{F} \cap X, m, T_{\mid J(T)}\right)$.

(2) Let

$$
\mathscr{R}(C, T)=\left\{\left[b_{1}, \ldots, b_{n}\right]: b_{n} \cap(J(T) \backslash B(\Lambda, \beta)) \neq \varnothing\right\},
$$

where $C=(k(1 / 2))^{h}$, with $k$ the constant appearing in the Köbe distortion theorem. Then $(T, \mathscr{R})$ has the Schweiger property with respect to $\mathscr{R}(C, T)$.

Lemma 9.5. (1) $(T, \mathscr{R})$ is parabolic with respect to $\mathscr{R}(C, T)$.

(2) The jump transformation $\left(T^{*}, \mathscr{R}^{*}\right)$ is aperiodic.

Proof. By the construction in Lemma 9.2, every point in $X$ has at most one preimage in $B(\Lambda, \beta)$. Therefore $\left.T\right|_{\left[N_{C} \geq 2\right]}$ is $1-1$ and $T\left(\left[N_{C}=1\right] \backslash T\left[N_{C}=\right.\right.$ 2]) $=X$, since $\left[N_{C} \geq 2\right] \subseteq B(\Lambda, \beta)$ and $(T, \mathscr{R})$ is parabolic.

It follows from Lemma 9.3 that there are two periodic points of prime periods whose orbits are contained in $\left[N_{C}=1\right]$, whence, by Theorem 7.1 , the jump transformation is aperiodic. $\diamond$

It now follows from Theorems 3.1, 3.2, and 4.2 that $(T, \mathscr{R})$ is conservative and exact, has a $\sigma$-finite invariant measure $\mu \sim m$, and has wandering rates. It follows from Theorem 6.1 that $\left(T^{*}, \mathscr{R}^{*}\right)$ has a finite invariant measure $q \sim m$, and is continued fraction mixing.

The next result establishes additional properties needed for the central limit theorem for $T$ (in case $\mu$ is finite), and the identification of wandering rates of $T$ (in case $\mu$ is infinite).

Definition. For $\mathscr{A}$ a partition of $J(T)$, let $C(\mathscr{A})$ denote the collection of bounded functions $f: J(T) \rightarrow \mathbb{R}$ which are uniformly continuous on $A^{o}$ for each $A \in \mathscr{A}$, and let $L(\mathscr{A})$ denote the collection of bounded functions $f$ : $J(T) \rightarrow \mathbb{R}$ which are uniformly Lipschitz continuous on $A^{o}$ (with respect to the spherical metric $d$ ) for each $A \in \mathscr{A}$.

If $\mathscr{A}$ is the Markov partition as above, then the above is nonvacuous as $\overline{A^{\circ}}=A$ for each $A \in \mathscr{A}$. We fix $\mathscr{A}$ as the Markov partition and denote $C=C(\mathscr{A})$, and $L=L(\mathscr{A})$. Let

$$
\|f\|_{C}=\max _{A \in \mathscr{A}} \sup _{x \in A^{o}}|f(x)|
$$

and

$$
\|f\|_{L}=\|f\|_{C}+\max _{A \in \mathscr{A}} \sup _{x, y \in A^{o}} \frac{|f(x)-f(y)|}{d(x, y)}
$$


then $\left(C,\|\cdot\|_{C}\right)$ and $\left(L,\|\cdot\|_{L}\right)$ are Banach spaces, $\bar{L}=C$, and bounded sets in $L$ are precompact in $C$ by the Arzéla-Ascoli theorem.

Definition. Let $S$ be a countable set. A function $f: S^{\mathbb{N}} \rightarrow \mathbb{R}$ is called Hölder continuous if

$$
\begin{aligned}
& \exists r \in(0,1), M>0 \ni \forall n \geq 1 \\
& x, y \in S^{\mathrm{N}}, b_{k}(x)=b_{k}(y) \quad(1 \leq k \leq n) \Rightarrow|f(x)-f(y)|<M r^{n} .
\end{aligned}
$$

Remark. By the bounded distortion properties used in the construction of $\mathscr{R}(C, T)$, we have that

$$
\exists t \in(0,1), M>0 \ni \max _{B \in \mathscr{R}_{0}^{* n}} \operatorname{diam} B<M t^{n} .
$$

Therefore, if $f \in L$, then $f \circ \pi^{*}$ is Hölder continuous on $\mathscr{R} * \mathbb{N}$, where $\pi^{*}: \mathscr{R}^{* \mathrm{~N}} \rightarrow J(T)$ is such that $b_{n}^{*}\left(\pi^{*}\left(y_{1}, \ldots\right)\right)=y_{n}$. Also, if $f \in L$, then $f \circ \pi$ is uniformly continuous on $\mathscr{R}^{\mathbb{N}}$, where $\pi: \mathscr{R}^{\mathbb{N}} \rightarrow J(T)$ is such that $b_{n}\left(\pi\left(y_{1}, \ldots\right)\right)=y_{n}$. This is because

$$
\max _{B \in \mathscr{R}_{0}^{n}} \operatorname{diam} B \rightarrow 0 \text { as } n \rightarrow \infty .
$$

Moreover, if $f \in L$ vanishes on some neighbourhood of $\Lambda$, and

$$
f^{(*)}(x)=f(x)+f(T x)+\cdots+f\left(T^{N_{C}(x)-1}(x)\right),
$$

then $f^{(*)} \circ \pi^{*}$ is bounded and Hölder continuous on $\mathscr{R}^{* \mathrm{~N}}$.

Theorem 9.6. (a) $\frac{d q}{d m} \in L$.

(b) $\left(T^{*}, \mathscr{R}^{*}\right)$ is continued fraction mixing with exponential rates.

Proof. By part (2) of Lemma 9.2, all inverses are defined and analytic in some neighbourhood of $J(T) \backslash B(\Lambda, \beta)$. For points in this neighbourhood, no preimage is a critical point of $T$. Thus the collection of inverse branches of $\left\{T^{n}\right\}_{n \in \mathbb{N}}$ forms a normal, and hence, equi-Lipschitz continuous, family in this neighbourhood with respect to the spherical metric. It follows from the Köbe distortion theorem that the logarithms of the absolute values of the spherical derivatives of the inverse branches of $\left\{T^{n}\right\}_{n \in \mathbb{N}}$ form an equi-Lipschitz continuous family in this neighbourhood. It follows from this that the family of inverse branches of $\left\{T^{n}\right\}_{n \in \mathrm{N}}$ and their Jacobians with respect to $m$ form an equi-Lipschitz continuous family on $\left[N_{C}=1\right]$.

Whence, the collection of inverse branches of $\left\{T^{* n}\right\}_{n \in \mathbb{N}}$ and their Jacobians also form an equi-Lipschitz continuous family on the interior of each set $A \in$ $\mathscr{A} \cap\left[N_{C}=1\right]$. Since $T^{*-1}\left[N_{C} \geq 2\right] \subset\left[N_{C}=1\right]$, and since the inverse branches of $T^{*}$ agree with inverse branches of $T$ on $\left[N_{C} \geq 2\right]$, it follows that the collection of inverse branches of $\left\{T^{* n}\right\}_{n \in \mathbb{N}}$ and their Jacobians form an equiLipschitz continuous family on the interior of each set $A \in \mathscr{A}$. This implies that if $f \in L$, then $\left\{\widehat{T}^{* n} f\right\}_{n \in \mathbb{N}}$ is a uniformly Lipschitz continuous family on the interior of each set $A \in \mathscr{A}$, and hence, by the Arzéla-Ascoli theorem, every subsequence has a subsequence converging in $C$ to a limit in $L$. It follows from this that there is a $T^{*}$-invariant probability $q \sim m$ such that $\frac{d q}{d m} \in L$. Recall from previous sections that $\log \frac{d q}{d m} \in L^{\infty}(m)$. 
Now let $\widehat{T}^{*}$ denote the dual operator to $f \mapsto f \circ T$ on $L^{1}(q)$. Specifically

$$
\widehat{T}^{*} f=\sum_{a \in \mathscr{R}^{*}} 1_{T^{*} a}\left|\tau_{a}^{\prime}\right|^{h} \frac{p \circ \tau_{a}}{p} f \circ \tau_{a},
$$

where $p=\frac{d q}{d m}$, and $\tau_{a}: T^{*} a \rightarrow a\left(a \in \mathscr{R}^{*}\right)$ are the inverse branches of $T^{*}$. Also, for $k \in \mathbb{N}$,

$$
\widehat{T}^{* k} f=\sum_{\mathbf{a} \in \mathscr{R}_{0}^{* k-1}} 1_{T^{*} a_{k}}\left|\tau_{\mathbf{a}}^{\prime}\right|^{h} \frac{p \circ \tau_{\mathbf{a}}}{p} f \circ \tau_{\mathbf{a}}
$$

where $\tau_{\mathbf{a}}: T^{*} a_{k} \rightarrow[\mathbf{a}]\left(\mathbf{a} \in \mathscr{R}_{0}^{* k-1}\right)$ are the inverse branches of $T^{*}$. To prove (b), we shall apply the theorem of Ionescu-Tulcea, and Marinescu [25]. To do this, we must show that

$$
\sup \left\{\frac{\left\|\widehat{T}^{* n} f\right\|_{C}}{\|f\|_{C}}: f \in L, n \in \mathbb{N}\right\}<\infty,
$$

and

(3) $\quad \exists r \in(0,1), R \in \mathbb{R}_{+}, k \in \mathbb{N} \ni\left\|\widehat{T}^{* k} f\right\|_{L} \leq r\|f\|_{L}+R\|f\|_{C} \quad \forall f \in L$.

Since, $\widehat{T}^{*} 1=1$, (1) is clear. As mentioned above, (2) follows from the ArzélaAscoli theorem. To prove (3), we note first that there is a number $M \in \mathbb{R}_{+}$ such that

$$
d(\tau x, \tau y) \leq M\left|\tau^{\prime}(x)\right| d(x, y)
$$

whenever $x, y \in A^{o} \cap(J(T) \backslash \Lambda), A \in \mathscr{A}$, and $\tau=\tau_{\mathrm{a}}$ is an inverse branch of $T^{* k}(k \in \mathbb{N})$. Using this, we see that

$$
\begin{aligned}
\frac{\left|\widehat{T}^{* k} f(x)-\widehat{T}^{* k} f(y)\right|}{d(x, y)} & \leq M_{1}\|f\|_{C}+M_{2}\|f\|_{L} \sum_{\mathbf{a} \in \mathscr{R}_{0}^{* k-1}}\left|\tau_{\mathbf{a}}^{\prime}(x)\right|^{1+h} \\
& \leq M_{1}\|f\|_{C}+M C^{6}\|f\|_{L} \frac{\sup \left\{q([\mathbf{a}]): \mathbf{a} \in \mathscr{R}_{0}^{* k-1}\right\}}{\min _{a \in \mathscr{R}} q(T a)} .
\end{aligned}
$$

Since

$$
\sup \left\{q([\mathbf{a}]): \mathbf{a} \in \mathscr{R}_{0}^{* k-1}\right\} \rightarrow 0 \text { as } k \rightarrow \infty
$$

and $\min _{a \in \mathscr{R}} q(T a)>0$, it follows that (3) is satisfied for some $k \in \mathbb{N}$.

As $T$ is exact, it follows from [25] that there exist $\rho \in(0,1)$ and $K \in \mathbb{R}_{+}$ such that

$$
\left\|\widehat{T}^{* n} f-\int_{X} f d q\right\|_{L} \leq K \rho^{n}\|f\|_{L}
$$

To deduce statement $(\mathbf{b})$ from this note that for $[\mathbf{a}]=\left[a_{1}, \ldots, a_{n}\right]$,

$$
\widehat{T}^{* n} 1_{[\mathbf{a}]}=1_{T * a_{n}}\left|\tau_{\mathbf{a}}^{\prime}\right| \frac{p \circ \tau_{\mathbf{a}}}{p},
$$

and, by the above,

$$
\sup \left\{\left\|1_{T * a_{k}} \frac{\left|\tau_{\mathbf{a}}^{\prime}\right|^{h}}{q([\mathbf{a}])}\right\|_{L}: k \in \mathbb{N},[\mathbf{a}]=\left[a_{1}, \ldots, a_{k}\right] \in \mathscr{R}_{0}^{* k-1}\right\}<\infty,
$$


whence

$$
M:=\sup \left\{\left\|\frac{\widehat{T}^{* n} 1_{[\mathbf{a}]}}{q([\mathbf{a}])}\right\|_{L}: n \in \mathbb{N}, \mathbf{a} \in \mathscr{R}_{0}^{* n-1}\right\}<\infty
$$

and

$$
\left\|\widehat{T}^{* n+k} 1_{[\mathbf{a}]}-q([\mathbf{a}])\right\|_{C} \leq K M \rho^{k} q([\mathbf{a}]) \quad \forall n, k \in \mathbb{N}, \mathbf{a} \in \mathbb{R}_{0}^{* n-1} .
$$

This is statement $(\mathrm{b}) . \diamond$

Remarks. (1) It follows from statement (a) that $\frac{d q}{d m}$ is uniformly continuous on $\mathbb{R}^{\mathbb{N}}$ with respect to the product topology.

(2) As a consequence of statement (b), we obtain that for any set $A \in$ $\mathscr{R}(C, T) \cap\left[N_{C}=1\right],\left(T_{A}, \mathscr{R}_{A}\right)$ is continued fraction mixing with exponential rates. This is because $T_{A}=T_{A}^{*}$, and $\mathscr{R}_{A}=\mathscr{R}_{A}^{*}$.

Recall that $P=\left[N_{C} \geq 2\right]$ is defined in $\S 5$ for parabolic fibred systems and that $f_{*}$ is the unique inverse branch of $T$ such that $f_{*}: T(P) \rightarrow P$. Clearly $f_{*}=T_{\omega}^{-1}$ on sufficiently small neighbourhoods of rationally indifferent periodic points $\omega$. Therefore, since $m$ is $h$-conformal, we obtain from Theorem 5.5 the following.

Proposition 9.7. Let $\mu$ be the $T$-invariant measure equivalent to the $h$-conformal measure $m$. Then $\mu$ is finite if and only if for some (every) $z \in P$

$$
\sum_{n=1}^{\infty} \frac{n}{\left|\left(T^{n}\right)^{\prime}\left(z_{n}\right)\right|^{h}}<\infty
$$

where $z_{n}=f_{*}^{n}(z)$.

For $\omega \in \Lambda$, let

$$
\alpha(\omega)=\frac{p(\omega)+1}{p(\omega)} h,
$$

where $p(\omega)$ is as in $\S 8$, and let

$$
\alpha=\min _{\omega \in \Lambda} \alpha(\omega) .
$$

Theorem 9.8. Let $T: \overline{\mathbb{C}} \rightarrow \overline{\mathbb{C}}$ be a parabolic rational map with $\sigma$-finite $T$ invariant measure $\mu \sim m$. Then the following assertions are true:

(1) If $\alpha>2$ then $\mu$ is finite.

(2) If $\alpha<2$ then there exists a constant $c_{\mu}>0$ such that

$$
\lim _{n \rightarrow \infty} \frac{L(n)}{n^{2-\alpha}}=c_{\mu}
$$

(3) If $\alpha=2$ then there exists a constant $c_{\mu}>0$ such that

$$
\lim _{n \rightarrow \infty} \frac{L(n)}{\log n}=c_{\mu}
$$

Proof. By Proposition 4.3 it suffices to prove this theorem for a positive iteration of $T$. In other words, we can assume that $\Lambda$ consists only of fixed points and $T^{\prime}(\omega)=1$ for every $\omega \in \Lambda$. By Theorem 4.1, $L(n) \sim L_{A}(n)$ for $A=\left[N_{C}=2\right]$. 
(1) follows from Proposition 9.7 and Theorem 8.4. By statement (a) of Theorem 9.6, Proposition 5.6 applies, and

$$
L(n) \sim \sum_{a \in T P \backslash P \cap \mathscr{R}} \sum_{\nu=0}^{d(a)-1} c_{a, \nu} \sum_{k=1}^{\infty}(k \wedge n) m\left(f_{*}^{d k+r+\nu} a\right) \quad \text { as } n \rightarrow \infty,
$$

where $c_{a, \nu}>0 \forall a, \nu$.

Clearly, for every $a \in T P \backslash P$ and $\nu, 0 \leq \nu \leq d(a)$, there exists $\omega_{a, \nu} \in \Lambda \ni$ $f_{*}^{d k+r+\nu}(x) \rightarrow \omega_{a, \nu} \forall x \in a$.

By Lemma $8.9 m\left(f_{*}^{d k+r+\nu} a\right) \sim \frac{c^{\prime}(a, \nu)}{k^{\alpha\left(\omega_{a}, \nu\right)}}$. Hence,

$$
\sum_{k=1}^{\infty}(k \wedge n) m\left(f_{*}^{d k+r+\nu} a\right) \sim \sum_{k=1}^{\infty}(k \wedge n) \frac{c^{\prime}(a, \nu)}{k^{\alpha\left(\omega_{a, \nu}\right)}} \sim c^{\prime \prime}(a, \nu) n^{2-\alpha\left(\omega_{a, \nu}\right)} \quad \text { as } n \rightarrow \infty .
$$

This proves $(2)$ of the theorem. $\diamond$

As an immediate consequence of this criterion we get the following

Theorem 9.9. If $h=H D(J(T)) \leq 1$ then the measure $\mu$ is infinite.

Theorem 9.10. If $h>1$ and $p(\omega)=1$ for every $\omega \in \Lambda$ then $\mu$ is finite.

Remarks. (1) If $B$ is a Blaschke product then $J(B) \subset S^{1}$, whence $h \leq 1$. By the Denjoy-Wolff theorem (see [11]), there is a fixed point $\omega \in \bar{U}$, the closed unit disc, such that $B^{n}(z) \rightarrow \omega$ for every $z \in U^{o}$ and $B^{n}(z) \rightarrow 1 / \bar{\omega}$ for every $z \in U^{c}$. $B$ has no critical points on $S^{1}$. Therefore, if $B^{\prime}(\omega)=1$ (and necessarily $\omega \in S^{1}$ ), then $B$ is parabolic and Theorem 9.9 applies. In case $B^{\prime \prime}(\omega)=0$ then $B$ is conservative with respect to Lebesgue measure on $S^{1}$. This follows for example from [1, Theorem 3.8]. Consequently $J(B)=S^{1}$, since otherwise $B^{n}(z) \rightarrow \omega$ as $n \rightarrow \infty$ for $z \in S^{1} \backslash J(B)$ and hence there would be wandering intervals for some power of $T$. Here, $\alpha=3 / 2$ and $p=2$. In this case, the exactness, pointwise dual ergodicity of $B$, and form of return sequence (established in Theorem 9.11 below) follow from [1, Theorem 3.8], which Theorem 9.11 strengthens and generalises.

(2) The simplest example satisfying the assumptions of Theorem 9.10 is provided by the polynomial $z \mapsto z-z^{2}$ (note that this map is conjugate by a linear map to $z \mapsto z+z^{2}$ and $\left.z \mapsto z^{2}+1 / 4\right)$. For $z \mapsto z-z^{2}, \Lambda=\{0\}$ and $p(0)=1$. Since the critical point $1 / 2$ is not in the Julia set, $z \mapsto z-z^{2}$ is parabolic. It is shown in $[39,37]$ that the Hausdorff dimension of its Julia set is greater than 1 .

Theorem 9.11. Suppose that

$$
\alpha=\min _{\omega \in \Lambda} \frac{p(\omega)+1}{p(\omega)} h \leq 2
$$

(hence the $T$-invariant measure $\mu$ is infinite). Then the dynamical system $(J(T), \mathscr{F}, \mu, T)$ is conservative and exact, has Darling-Kac sets whose return time processes are continued fraction mixing with exponential rates, and has a return sequence given by

$$
a_{n}(T) \sim \begin{cases}\frac{1}{c_{\mu} \Gamma(\alpha) \Gamma(3-\alpha)} n^{\alpha-1} & \text { when } 1<\alpha<2, \\ \frac{1}{c_{\mu}} \frac{n}{\log n} & \text { when } \alpha=2 .\end{cases}
$$

Here $c_{\mu}$ is as in Theorem 9.8 . 
Proof. By Lemma 9.5, $(T, \mathscr{R})$ is an aperiodic, parabolic, finite fibred system with respect to $\mathscr{R}(C, T)$. By Theorem 2.8,T is conservative and ergodic. By Theorem 3.2, $T$ is exact. By Theorem 3.1, any set in $\mathscr{R}(C, T)$ is a DarlingKac set for $T$ whose return time process is continued fraction mixing. By Theorem 9.8 the wandering rates $L(n)$ satisfy

$$
L(n) \sim \begin{cases}c_{\mu} n^{2-\alpha} & (1<\alpha<2), \\ c_{\mu} \log n & (\alpha=2)\end{cases}
$$

as $n \rightarrow \infty$. In the present situation, as explained in $\S 4$, Theorem 3 in [2] or the argument in [3, p. 1044], combined with Karamata's Tauberian theorem shows that

$$
a_{n}(T) \sim \frac{n}{L(n)} \frac{1}{\Gamma(1+\gamma) \Gamma(2-\gamma)},
$$

where $\gamma=2-\alpha$. This implies the theorem. $\diamond$

The following two results concerns the central limit theorem in case of a finite invariant measure. We do not know whether such a result holds for all Hölder continuous functions.

Theorem 9.12. Let $\mu$ be finite $(\alpha>2)$. Then for any Hölder continuous function $f: J(T) \rightarrow \mathbb{R}$ which vanishes on some neighbourhood of $\Lambda$ and satisfies $\mu(f)=$ 0 ,

$$
c_{f}=q\left(\left(f^{*}\right)^{2}\right)+2 \sum_{l=1}^{\infty} q\left(f^{*} f^{*}\left(T^{*}\right)^{l}\right)<\infty
$$

converges absolutely, and if $c_{f}>0$ then $f$ satisfies the central limit theorem:

$$
\begin{gathered}
\lim _{n \rightarrow \infty} \mu\left(\left[\frac{\sqrt{\mu(J(T))}}{\sqrt{n c_{f}}} \sum_{l=0}^{n}\left(f \circ T^{l}-\frac{\mu(f)}{\mu(J(T))}\right) \leq t\right]\right) \\
=\frac{\mu(J(T))}{\sqrt{2 \pi}} \int_{-\infty}^{t} \exp \left(\frac{-u^{2}}{2}\right) d u
\end{gathered}
$$

for any $t \in \mathbb{R}$.

Proof. By (b) in Theorem 9.6, $\left(T^{*}, \mathscr{R}^{*}\right)$ is continued fraction mixing with exponential mixing rates. By the remarks before Theorem $9.6, f^{*}$ is bounded and Hölder continuous on $\mathscr{R}{ }^{* N}$. Hence Theorem 7.2 implies the theorem. $\diamond$

Theorem 9.13. Let $\mu$ be finite, and let $f$ be measurable with respect to $\mathscr{R}_{0}^{m}$ for some $m \geq 1$ such that $f^{*} \in L^{2}(q)$. If $\sigma_{n}^{2}(f) \rightarrow \infty$ as $n \rightarrow \infty$, then

$$
\sigma_{n}^{2}(f)=n h(n)
$$

where $h(n)$ is slowly varying as $n \rightarrow \infty$, and $f$ satisfies the central limit theorem:

$$
\begin{aligned}
\lim _{n \rightarrow \infty} \mu & \left(\left[\frac{\sqrt{\mu(J(T))}}{\sigma_{n}(f)} \sum_{l=0}^{n}\left(f \circ T^{l}-\frac{\mu(f)}{\mu(J(T))}\right) \leq t\right]\right) \\
= & \frac{\mu(J(T))}{\sqrt{2 \pi}} \int_{-\infty}^{t} \exp \left(\frac{-u^{2}}{2}\right) d u
\end{aligned}
$$

for any $t \in \mathbb{R}$. 


\section{Proof. This follows immediately from Corollary 7.3. $\diamond$}

Remark. Theorem 9.12 is true for all functions $f$ with the property that $f^{*}$ is bounded and Hölder continuous on $\mathscr{R} * \mathrm{~N}$.

\section{REFERENCES}

1. J. Aaronson, Ergodic theory for inner functions of the upper half plane, Ann. Inst. H. Poincaré Sec. B 14 (1978), 233-253.

2. The asymptotic distributional behaviour of transformations preserving infinte measure J. Analyse Math. 39 (1981), 203-234.

3. __, Random f-expansions, Ann. Probab. 14 (1986), 1037-1057.

4. J. Aaronson and M. Denker, Upper bounds for ergodic sums of infinite measure preserving transformations, Trans. Amer. Math. Soc. 319 (1990), 101-138.

5. __ Lower bounds for partial sums of certain positive stationary processes, Almost Everywhere Convergence, Proceedings (G. A. Edgar and L. Sucheston, eds.), Academic Press, 1989, pp. 1-9.

6. mations, C.R. Acad. Sci. Paris 313 (1991), 471-475.

7. J. Aaronson, M. Denker, and A. Fisher, Second order ergodic theorems for ergodic transformations of infinite measure spaces, Proc. Amer. Math. Soc. 114 (1992), 115-127.

8. P. Blanchard, Complex analytic dynamics of the Riemann sphere, Bull. Amer. Math. Soc. (N. S.) 11 (1984), 85-141.

9. R. Bradley, On the $\psi$-mixing condition for stationary random sequences, Trans. Amer. Math. Soc. 276 (1983), 55-66.

10. H. Brolin, Invariant sets under iteration of rational functions, Ark. Mat. 6 (1965), 103-144.

11. A. Denjoy, Fonctions contractent le cercle $|z|<1$, C. R. Acad. Sci. Paris 182 (1926), 255-257.

12. M. Denker, Some new examples of Gibbs measures, Monatsh. Math. 109 (1990), 49-62.

13. M. Denker, C. Grillenberger, and K. Sigmund, Ergodic theory on compact spaces, Lecture Notes in Math., vol. 527, Springer-Verlag, Berlin-Heidelberg-New York, 1976.

14. M. Denker and M. Urbański, Absolutely continuous invariant measures for expansive rational maps with rationally indifferent periodic points, Forum Math. 3 (1991), 561-579.

15. __ Hausdorff and conformal measures on Julia sets with a rationally indifferent periodic point, J. London Math. Soc. 43 (1991), 107-118.

16. __ On Sullivan's conformal measures for rational maps of the Riemann sphere, Nonlinearity 4 (1991), 365-384.

17. R. Devaney, An introduction to chaotic dynamical systems, Benjamin, 1985.

18. A. Douady and J. H. Hubbard, Etude dynamique des polynomes complexes, Publ. Math. Orsay Univ. Paris XI, Orsay, 1985.

19. P. Fatou, Sur les equations fonctionnelles, Bull. Soc. Math. France 47 (1919), 161-271.

20. Sur les equations fonctionnelles, Bull. Soc. Math. France 48 (1920), 33-94.

21. M. Guzmán, Differentiation of integrals in $\mathbb{R}^{n}$, Lecture Notes in Math., vol. 481, SpringerVerlag.

22. E. Hille, Analytic function theory, Ginn, Boston, Mass., 1962.

23. F. Hofbauer and G. Keller, Ergodic properties of invariant measures for piecewise monotonic transformations, Math. Z. 180 (1982), 119-140.

24. I. A. Ibragimov and Y. V. Linnik, Independent and stationary sequences of random variables, Wolters-Noordhoff, Groningen, 1971.

25. C. Ionescu-Tulcea and G. Marinescu, Théorie ergodique pour des classes d'opérations non completement continues, Ann. of Math. (2) 52 (1950), 140-147. 
26. M. Kac, On the notion of recurrence in discrete stochastic processes, Bull. Amer. Math. Soc. 53 (1947), 1002-1010.

27. S. Kakutani, Induced measure preserving transformations, Proc. Imperial Acad. Sci. Tokyo 19 (1943), 635-641.

28. L. Keen, Julia sets, Chaos and Fractals (R. Devaney and L. Keen, eds.), Proc. Sympos. Appl. Math., vol. 39, Amer. Math. Soc. Providence, R.I., 1989, pp. 57-74.

29. U. Krengel, Ergodic theorems, De Gryuter, Berlin and New York, 1985.

30. A. Renyi, Representations for real numbers and their ergodic properties, Acta Math. Acad. Sci. Hungar. 8 (1957), 477-493.

31. D. Ruelle, Thermodyamic formalism, Encyclopedia Math. Appl., vol. 5, Addison-Wesley, Reading, Mass., 1976.

32. M. Rychlik, Bounded variation and invariant measures, Studia Math. 76 (1983), 69-80.

33. F. Schweiger, Number theoretical endomorphisms with $\sigma$-finite invariant measures, Israel J. Math. 21 (1975), 308-318.

34. D. Sullivan, Conformal dynamical systems, Geometric Dynamics, Lecture Notes in Math., vol. 1007, Springer-Verlag, 1983, pp. 725-752.

35. M. Thaler, Estimates of the invariant densities of endomorphisms with indifferent fixed points, Israel J. Math. 37 (1980), 303-314.

36. _ Transformations on $[0,1]$ with infinite invariant measures, Israel J. Math. 46 (1983), 67-96.

37. M. Urbański, On Hausdorff dimension of the Julia set with a rationally indifferent periodic point, Studia Math. 97 (1991), 167-188.

38. M. Yuri, On a Bernoulli property for multidimensional mappings with finite range structure, Tokyo J. Math. 9 (1986), 457-485.

39. A. Zdunik, Parabolic orbifolds and the dimension of the maximal measure for rational maps, Invent. Math. 99 (1990), 627-649.

School of Mathematical Sciences, Tel Aviv University, Ramat Aviv, 69978 Tel Aviv, ISRAEL

Institut für Mathematische Stochastik, Georg-August-Universität, LotZestr. 13 , 3400 GÖTTINGEN, GERMANY

Instytut Matematyki UMK, Ul. Chopina 12/18, 87-100 ToRuń, Poland

Current address: Department of Mathematics, North Texas University, Denton, Texas 762035116 